\title{
A MULTIBIT REFERENCE FEEDBACK SIGMA-DELTA MODULATOR FOR RADIO RECEIVERS
}

by

\author{
WENSHENG V. KUANG
}

A Thesis Submitted to the Faculty of Graduate Studies and Research In partial fulfillment of the requirements for the degree of Doctor of Philosophy

\author{
Ottawa-Carleton Institute for Electrical and Computer Engineering \\ Department of Electronics \\ Carleton University \\ Ottawa, Ontario
}

February 2007

(C) COPYRIGHT

2007, W. V. KUANG 


$\begin{array}{ll}\begin{array}{l}\text { Library and } \\ \text { Archives Canada }\end{array} & \begin{array}{l}\text { Bibliothèque et } \\ \text { Archives Canada }\end{array} \\ \begin{array}{l}\text { Published Heritage } \\ \text { Branch }\end{array} & \begin{array}{l}\text { Direction du } \\ \text { Patrimoine de l'édition }\end{array} \\ \begin{array}{l}\text { 395 Wellington Street } \\ \text { Ottawa ON K1A ON4 }\end{array} & \begin{array}{l}\text { 395, rue Wellington } \\ \text { Ottawa ON K1A ON4 } \\ \text { Canada }\end{array}\end{array}$

Your file Votre référence ISBN: 978-0-494-27101-8 Our file Notre référence ISBN: 978-0-494-27101-8

NOTICE:

The author has granted a nonexclusive license allowing Library and Archives Canada to reproduce, publish, archive, preserve, conserve, communicate to the public by telecommunication or on the Internet, loan, distribute and sell theses worldwide, for commercial or noncommercial purposes, in microform, paper, electronic and/or any other formats.

The author retains copyright ownership and moral rights in this thesis. Neither the thesis nor substantial extracts from it may be printed or otherwise reproduced without the author's permission.
AVIS:

L'auteur a accordé une licence non exclusive permettant à la Bibliothèque et Archives Canada de reproduire, publier, archiver, sauvegarder, conserver, transmettre au public par télécommunication ou par l'Internet, prêter, distribuer et vendre des thèses partout dans le monde, à des fins commerciales ou autres, sur support microforme, papier, électronique et/ou autres formats.

L'auteur conserve la propriété du droit d'auteur et des droits moraux qui protège cette thèse. $\mathrm{Ni}$ la thèse ni des extraits substantiels de celle-ci ne doivent être imprimés ou autrement reproduits sans son autorisation.
In compliance with the Canadian

Privacy Act some supporting forms may have been removed from this thesis.

While these forms may be included in the document page count, their removal does not represent any loss of content from the thesis.
Conformément à la loi canadienne sur la protection de la vie privée, quelques formulaires secondaires ont été enlevés de cette thèse.

Bien que ces formulaires aient inclus dans la pagination, il n'y aura aucun contenu manquant.

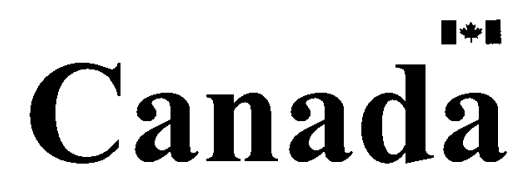




\section{ABSTRACT}

A reference feedback multibit sigma-delta modulator (SDM) for $A / D$ conversion in a radio receiver is studied in this thesis. The study shows that the reference feedback SDM is naturally signal-matched over process and temperature variations, which offers considerable advantage to reference circuit design and allows intentional dynamic range \& AGC control, an advantage that is not available to a conventional feedback SDM loop and is not practical for a Nyquist-rate A/D converter. A $4^{\text {th }}$-order 3-bit bandpass continuous-time reference feedback SDM is designed, built and experimentally evaluated.

A multibit SDM has a wider bandwidth than its 1-bit counterpart. Quantitative comparison of multibit vs 1-bit SDMs is conducted in terms of dynamic range. It shows that with a moderate level of D/A element mismatch, the multibit SDM is better than a 1bit SDM that inherently has no element mismatch, for bandwidths above $500 \mathrm{kHz}$. It suggests that a multibit SDM without using dynamic element matching (DEM) can still be a viable solution for broadband applications.

A novel biquad circuit is also developed for a continuous-time SDM loop filter. By cascading the novel biquad with regular biquad circuits, any higher order loop filter can be easily designed and implemented, considerably easing higher order continuoustime SDM design and prototyping. The novel biquad is implemented in the $4^{\text {th }}$-order loop filter design and experimentally demonstrated. 


\section{ACKNOWLEDGEMENTS}

I would like to thank my supervisor Prof. Jim Wight for giving me guidance and advice in this research. This work has been mostly carried out off-campus, yet he has always been there when help is needed.

I would also like to thank the committee members Profs. S. Haykin, S. Loyka, C. Plett, and R. Hafez for their valuable time and recommendations.

My wife Jie, my daughter Ying, and my son Andy played important roles in this work. I am pretty sure that without them I could have finished the work a lot sooner, but I am also equally sure that life would be a lot less joyful.

Finally, I wish to thank Samsung Telecom of America for their valuable support in the last phase of this research work. 


\section{Table of Contents}

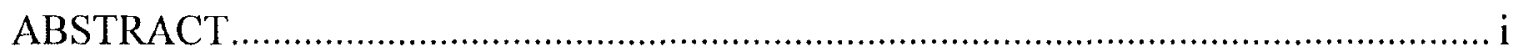

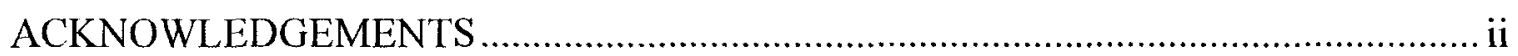

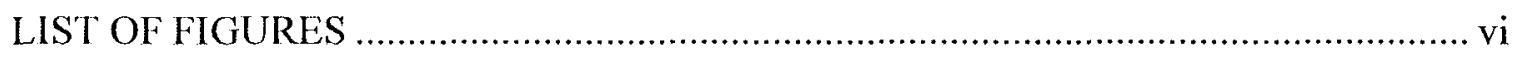

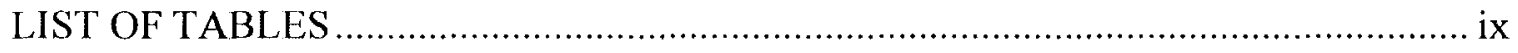

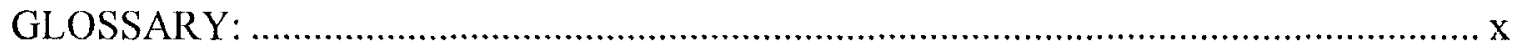

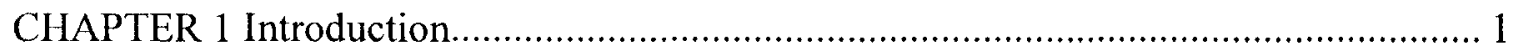

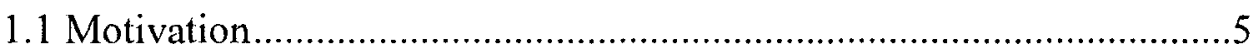

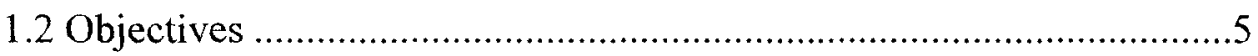

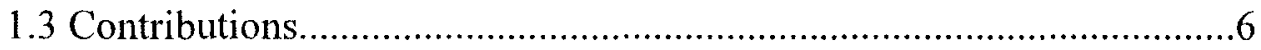

CHAPTER 2 Radio Receiver Review ................................................................. 8

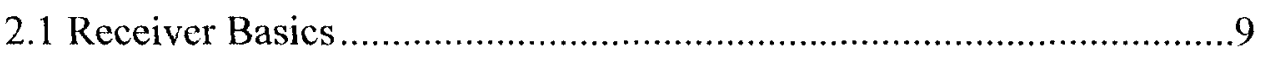

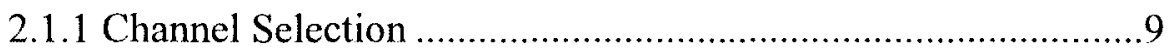

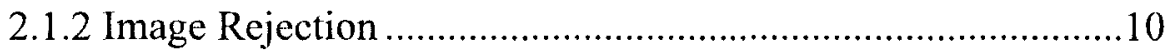

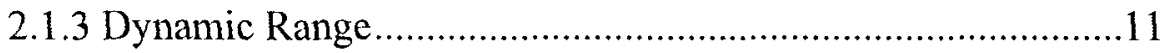

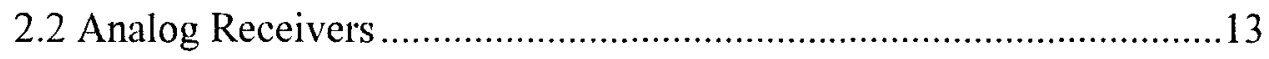

2.2.1 Conventional Super-Heterodyne .....................................13

2.2.2 Integrated Super-Heterodyne ......................................... 14

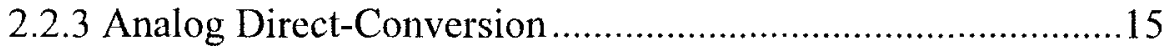

2.3 Digital IF Receivers ............................................................. 17

2.3.1 Wideband Digital IF Receiver .........................................17

2.3.2 Narrowband Digital Low-IF Receiver ................................19

2.3.3 Sub-Sampling Receiver ...........................................21

2.3.4 Bandpass Oversampling Receiver ..................................22

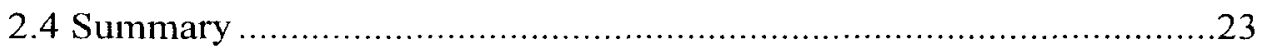

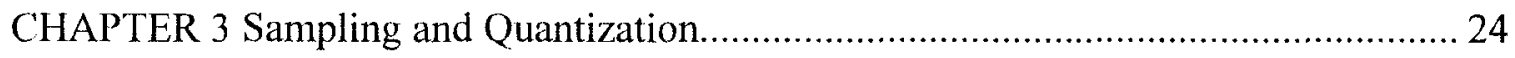

3.1 Ideal and Natural Sampling ....................................................24

3.2 Flat-top Sampling and the Aperture Effect.....................................26

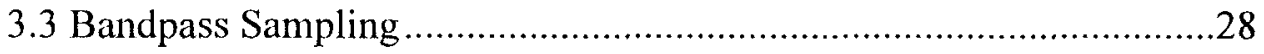


3.3.1 Quadrature Sampling for Direct-Conversion..........................30

3.3.2 Bandpass Sampling as Down-Conversion .............................33

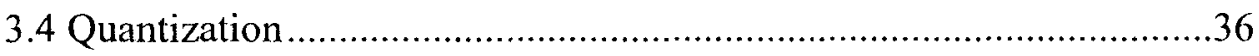

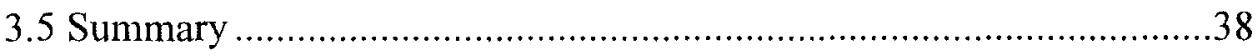

CHAPTER 4 Digital Filtering and Sampling Rate Conversion.....................................39

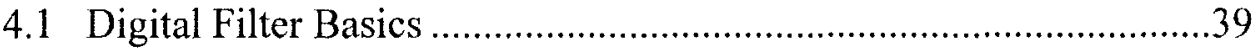

4.2 Sampling Rate Conversion Filtering............................................43

4.2.1 Decimation Filter --- Down-Sampling by an Integer Factor ...44

4.2.2 Interpolation Filter --- Up-Sampling by an Integer Factor ......47

4.2.3 Sampling Rate Conversion by a Rational Factor ....................50

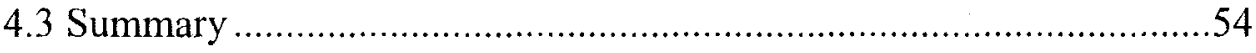

CHAPTER 5 Noise Shaped Oversampling - Sigma-Delta Modulator ............................ 55

5.1 Stability of the Nonlinear Feedback Loop .......................................57

5.2 Linearity of the Nonlinear Feedback Loop...................................58

5.3 1-Bit Sigma-Delta Modulator .........................................................60

5.3.1 Multi-Stage SDM (MASH) .......................................62

5.3.2 Reduced Gain for Stability..............................................63

5.4 Multibit Sigma-Delta Modulator .................................................65

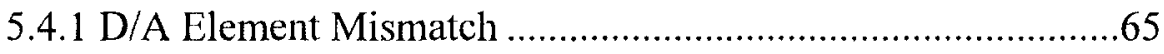

5.4.2 Dynamic Element Matching (DEM) ...................................66

5.5 A Quantitative Comparison of 1-Bit and Multi-Bit SDM .................68

5.6 Multibit Reference Feedback SDM ...............................................71

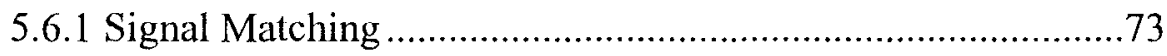

5.6.2 Thermal Noise Consideration ..........................................77

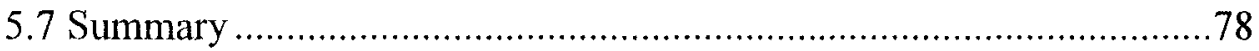

CHAPTER 6 Design of a Reference Feedback SDM............................................... 79

6.1 Loop Filter Transfer Function for CT SDM ....................................81

6.2 A Novel Cascaded Loop Filter ....................................................84

6.3 Design of the $4^{\text {th }}$-order CT Loop Filter ...........................................86

6.4 Transmission Gate Switch .........................................................88

6.5 Circuit Simulation.................................................................... 90

$$
\text { - iv - }
$$


6.6 Summary . .91

CHAPTER 7 Experimental Prototype of the Designed SDM ................................... 92

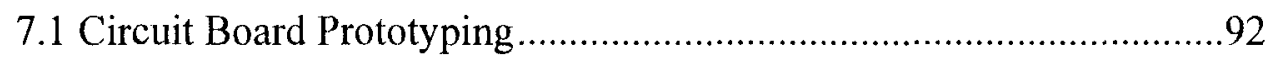

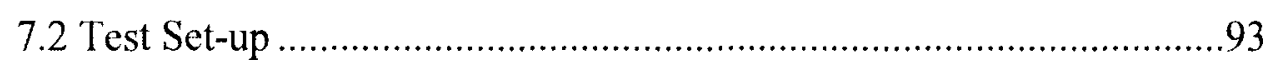

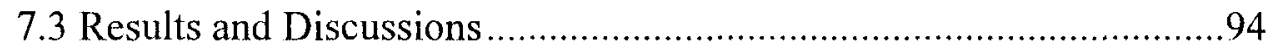

7.3.1 Test of a Varying Reference Voltage.................................98

7.3.2 Noise-Shaping Comparison with Conventional Feedback ....100

7.3.3 SNR Comparison with Conventional Feedback ...................102

7.3.3 Two-Tone Test of the Reference Feedback SDM ................104

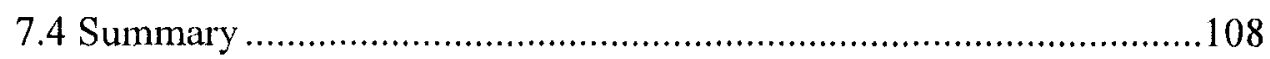

CHAPTER 8 Conclusions and Future Work ........................................................ 109

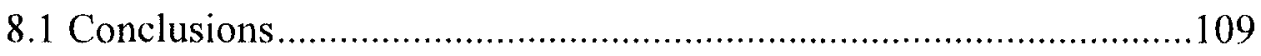

8.2 Future Work .........................................................................110

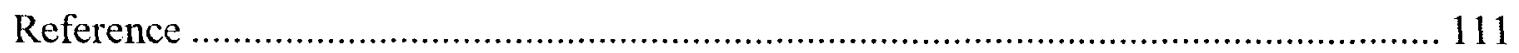

Appendix A Quadrature Sampling Alias-Free Conditions ................................... 117

Appendix B A $4^{\text {th }}$-order CT Loop Filter Transfer Function ...................................... 120

Appendix C Pulse Invariant Transformation Pair for QRZ DAC ............................. 123 


\section{LIST OF FIGURES}

Fig. 1.1 Digital Radio (Wireless) Communications....................................................... 1

Fig. 1.2 A Digital Radio Using Wideband A/D and NCO........................................... 2

Fig. 1.3 A Radio with Analog Front-End and Narrowband A/D at Baseband. ................. 3

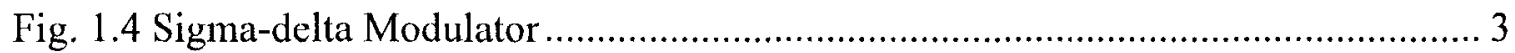

Fig. 2.1 A Functional Block Diagram of Digital Radio Receiver. .................................... 9

Fig. 2.2 Image Rejection by Pre-filtering Before Mixing............................................. 10

Fig. 2.3 Image Post-Cancelling After Mixing …………........................................... 11

Fig. 2.4 A Dual-Conversion Super-Heterodyne Radio Receiver...................................... 13

Fig. 2.5 A Highly Integrated Super-Heterodyne Radio Receiver................................... 15

Fig. 2.6 A Direct-Conversion Radio ........................................................................... 16

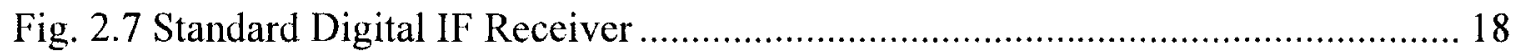

Fig. 2.8 A Low-IF Receiver with Complex Filter Prior to A/D Conversion.................... 19

Fig. 2.9 A Low-IF Receiver with A/D Converters Before Image Rejection.................. 20

Fig. 2.10 A Quadrature Sampling Software-Defined Radio Architecture-....................... 20

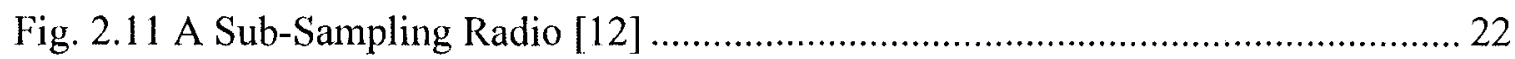

Fig. 3.1 Ideal Sampling ...................................................................................... 24

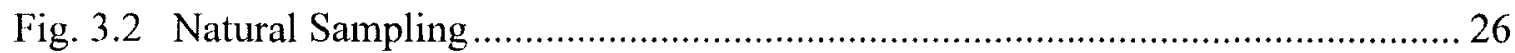

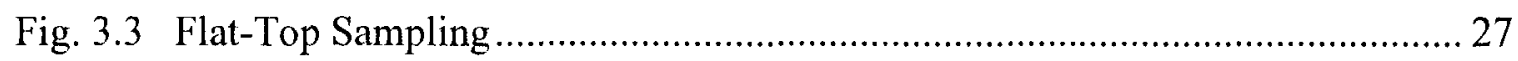

Fig. 3.4 Spectrum of (a) a Bandpass Signal and (b) its Sampled Signal ........................ 28

Fig. 3.5 Alias-free Sampling Zones (white) for Both Lowpass and Bandpass Sampling 30

Fig. 3.6 Quadrature Sampling Image Location.............................................................. 31

Fig. 3.7 Spectrum of Sampled Bandpass Signal For $\mathrm{K}=2 \mathrm{~N}$..................................... 34

Fig. 3.8 Spectrum of Sampled Bandpass Signal for $\mathrm{K}=2 N+1$..................................... 35

Fig. 3.9 Mid-Rise Quantizer (left) and Mid-Tread Quantizer (right) .............................. 37

Fig. 4.1 Tapped-Delay-Line (Transversal) Model of a Digital Filter ............................... 41

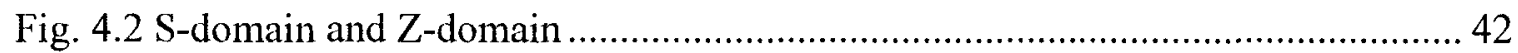

Fig. 4.3 A Decimate-by-Three Polyphase Filter Implementation................................... 45 
Fig. 4.4 A Single-Stage CIC Filter Structure …………............................................. 46

Fig. 4.5 A Single-Stage CIC Structure with a Down Sampler......................................... 46

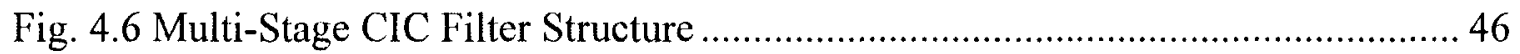

Fig. 4.7 Spectral Impact of Interpolate-by-Three Using Zero Padding ............................ 47

Fig. 4.8 Tapped-Delay-Line (Transversal Model) for Interpolation Digital Filter ........... 48

Fig. 4.9 A Interpolate-by-Three Polyphase Filtering Implementation ............................ 49

Fig. 4.10 Direct-Form by A Rational Factor $L / D$....................................................... 50

Fig. 4.11 An Efficient Way of Sampling Rate Conversion by A Rational Factor I/D .... 51

Fig. 4.12 Polyphase on Interpolator for Sampling Rate Conversion by A Rational Factor

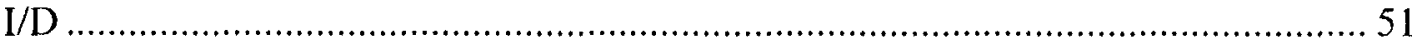

Fig. 4.13 The Most Efficient Way of Sampling Rate Conversion by A Rational Factor I/D

Fig. 4.14 Clock Structural Model for the Most Efficient Way of Sampling Rate

Conversion by a Rational Factor I/D

Fig. 4.15 An Example of Sampling Rate Conversion by 5/3 in Comparison with That by

$5 / 1$ 53

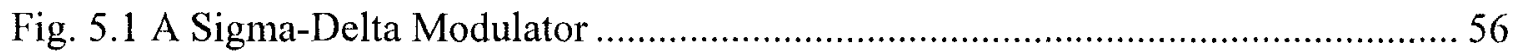

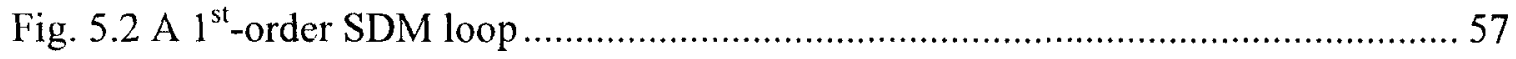

Fig. 5.3 Linear Model for a Sigma-delta Modulator (SDM) ……..................................... 60

Fig. 5.4 Noise-Shaping of a 1-bit First-order Lowpass SDM.......................................... 61

Fig. 5.5 A Multi-Stage (MASH) Lowpass Sigma-delta Modulator................................... 62

Fig. 5.6 An $m$ th-order Lowpass Sigma-delta Modulator ................................................. 64

Fig. 5.7 Sixth-order Bandpass SDM NTF Full-Gain $\left(1+z^{-2}\right)^{3}$ (dotted line) and Reduced-

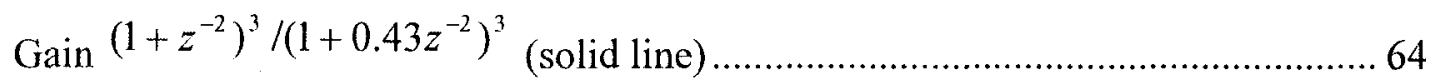

Fig. 5.8 A 5-level D/A Converter Incorporated in The First Integrator of a SDM........... 66

Fig. 5.9 A Multi-bit SDM with Dynamic Element Matching (DEM) ........................... 67

Fig. 5.10 Maximum Signal Output Spectrum of 1-bit SDM vs. 3-bit SDM of 0.5\%

Mismatch under $1.3 \mu \mathrm{V}$ Noise Voltage 69

Fig. 5.11 Direct Feedback of the Reference versus Conventional Feedback of the Digital Output .71 
Fig. 5.12 An Example of Reference Feedback SDM............................................... 72

Fig. 5.13 Noise Transfer Function for Different Loss Factor in the Loop........................ 74

Fig. 5.14 Drop of Maximum Input Level with Changing Reference............................... 75

Fig. 5.15 Noise-Shaping Spectrum for the Conventional SDM with a Reference Voltage

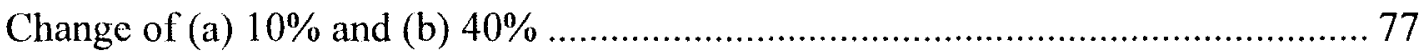

Fig. 6.1 Continuous-Time (CT) Sigma-Delta Modulator ……………………….......... 81

Fig. 6.2 Zero-Order-Hold DAC Impulse Response ……......................................... 82

Fig. 6.3 Full-Gain NTF $=\left(1+z^{-2}\right)^{2}$ of a $4^{\text {th }}$-order Bandpass SDM ............................... 83

Fig. 6.4 A New Biquad Circuit for CT SDM Loop Filter............................................. 85

Fig. 6.5 Cascaded Loop Filter with the New Biquad..................................................... 87

Fig. 6.6 Loop Filter Simulation and Measurement ..................................................... 88

Fig. 6.7 A Switch Matrix of CMOS Gates for The Reference Feedback SDM .............. 89

Fig. 6.8 A Simple XOR Gates for Switch Control ………………………................... 89

Fig. 6.9 Simulated Circuit for the New SDM ................................................................ 90

Fig. 6.10 One of the Circuit Simulation Results ............................................................. 90

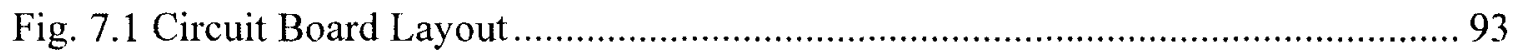

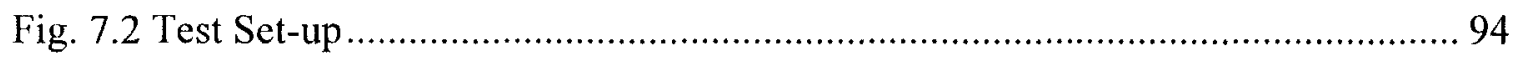

Fig. 7.3 Snapshot of The SDM Output Waveform (Top); Bottom Trace is The Clock ... 94

Fig. 7.4 An Example of the Digitized Data (100MHz) prior to Decimation .................... 95

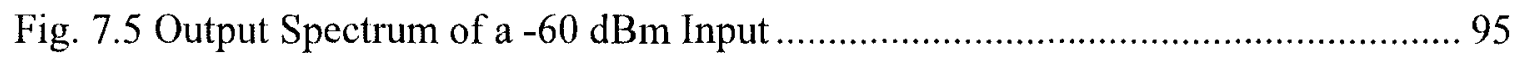

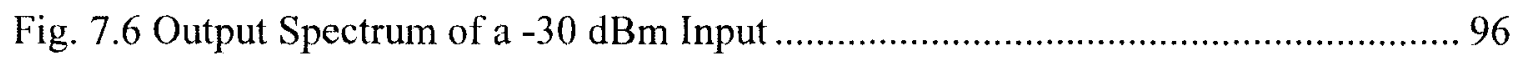

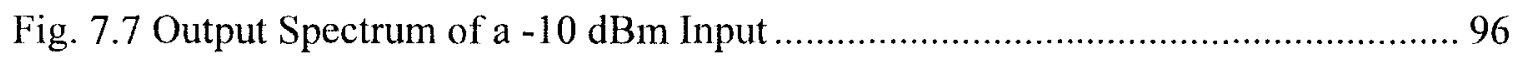

Fig. 7.8 SNR over $500 \mathrm{kHz}$ Bandwidth for the SDM Prototype ..................................... 97

Fig. 7.9 Noise-Shaping Spectrum with the Reference Being 0.7 volt ............................ 98

Fig. 7.10 Noise-Shaping Spectrum with the Reference Being 1.5 volt ........................... 99

Fig. 7.11 Noise-Shaping Spectrum with the Reference Being 3 volt .............................. 99

Fig. 7.12 Output Spectrum of the SDM with a Conventional DAC, Ref. $=1$ volt........ 100

Fig. 7.13 Output Spectrum of the SDM with a Conventional DAC, Ref. $=0.5$ volt..... 101

Fig. 7.14 Output Spectrum of the SDM with a Conventional DAC, Ref. $=2$ volt......... 101

Fig. 7.15 Output Spectrum of the SDM with a Conventional DAC, $-30 \mathrm{dBm}$ Input .... 102

Fig. 7.16 Output Spectrum of the SDM with a Conventional DAC, $-50 \mathrm{dBm}$ Input .... 103 


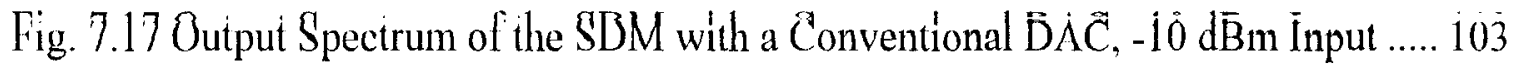

Fig. 7.18 SNR Plot of the Conventional DAC SDM versus the New SDM.................. 104

Fig. 7.19 Two-Tone Test with a Tone Spacing of $97656 \mathrm{~Hz}$........................................ 105

Fig. 7.20 Two-Tone Test with a Tone Spacing of $195312 \mathrm{~Hz}$..................................... 105

\section{LIST OF TABLES}

Table 2.1 A Simplified Comparison amongst the Receiver Architectures....................... 23

Table 4.1 Efficiency of the Skipped Polyphase Interpolator ........................................... 54

Table 5.1. Simulation Conditions for the Two SDMs .................................................. 70

Table 5.2. Simulation Results for SNR (signal-to-noise ratio) for $500 \mathrm{kHz}$ Bandwidth.. 70

Table 5.3. Simulation Results for SNR for $200 \mathrm{kHz}$ Bandwidth................................... 70

Table 7.1 Components Used for the 3-bit Reference Feedback SDM............................. 92 


\section{GLOSSARY:}

$\mathrm{A} / \mathrm{D} \quad$ analog to digital converter

ADC analog to digital converter

AGC automatic gain control

BIBO bound input bounded output

BPF band pass filter

CAD computer aided design

CIC cascaded integrator-comb

CT continuous time

CTSDM continuous time sigma-delta modulator

$\mathrm{D} / \mathrm{A}$ digital to analog converter

DAC digital to analog converter

DEM dynamic element matching

DT discrete time

DTSDM discrete time sigma-delta modulator

FIR finite impulse response

GBW gain bandwidth

IF intermediate frequency

IIR infinite impulse response

ISI Inter-symbol interference

LO local oscillator

LPF low pass filter

LUT look-up table

MACS Multiply-Accumulates per Second

$\mathrm{NCO}$ numerically controlled oscillator

NTF noise transfer function

PLL phase-locked loop

PSD power spectral density 
Q quality, quality factor

$\mathrm{RC} \quad$ resistance capacitance

$\mathrm{RF} \quad$ radio frequency

RMS root mean square

SAW Surface acoustic wave

SC switched capacitor

SDM sigma-delta modulator

SDR software-defined radio

SNR signal-to-noise ratio

STF signal transfer function 


\section{CHAPTER 1 Introduction}

The digital revolution has changed the way of communication in the world. Wireless radio communications had also evolved over the last century from analog to digital. By digital we mean that information is coded and transmitted in the form of digital bits, and signal processing is preferably performed by digital circuits and/or accompanying soltware. In a sense, one can say that the digital revolution in wireless communications is well developed. However, wireless communications differs from wired communications in that the information, even in the form of digital bits, still has to be carried by an analog radio frequency (RF) wave in order to be transmitted over the air. On the receiving side, the digital bits are recovered or demodulated from the RF carrier. The scenario is illustrated in Figure 1.1.

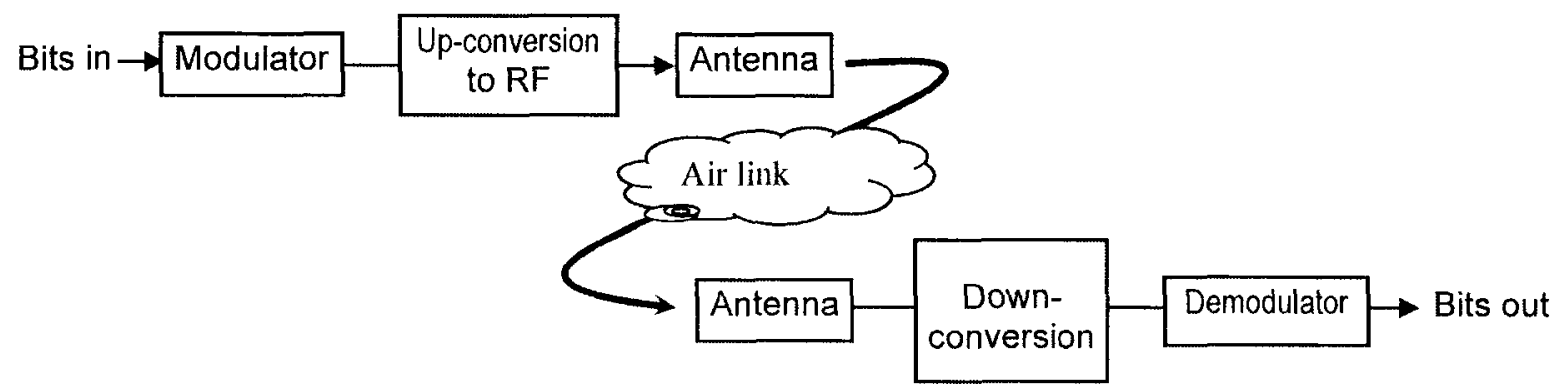

Fig. 1.1 Digital Radio (Wireless) Communications

Therefore the signal and its processing must be analog at least in some of the stages. For example, from antenna to antenna, it will probably stay analog forever. Digital radio is not entirely digital, rather a mix of analog and digital. A/D and D/A conversions are necessary between the analog and digital worlds. It has been common practice to place the $D / A$ and $A / D$ in the baseband portions of the transmitter and receiver where the signal is narrowband in frequency and slowly changing in time. Recently, effort has been made to move the $D / A$ and $A / D$ to IF frequencies. Technological advancement is often motivated by economics. By moving the $\mathrm{D} / \mathrm{A}$ and $\mathrm{A} / \mathrm{D}$ to IF frequencies, a radio can be 
made more flexible and efficient (i.e. more functions can be performed with less hardware). However, it proves quite challenging to process IF radio signals digitally, not only for $\mathrm{A} / \mathrm{D}$ conversion itself but also for subsequent digital signal processing. The challenges are trade-offs of conflicting goals among cost, size, power consumption, and performance in dealing with wideband and fast-changing signals. It is no surprise that most digital radios are still analog in the RF/IF stages.

A second difficulty arises from the multi-channel, wideband nature of radio signals at RF/IF. Since most radios work on multiple channels, even though only one channel is selected at a time, an A/D converter with a high dynamic range or a large number of bits is required to sample over a wide bandwidth. However, wideband A/D conversion is not only expensive in itself, but it also puts pressure on subsequent digital signal processing stages in terms of memory size and the number of multiplications performed per second. An example of such a wideband digital radio is illustrated in Figure 1.2. In the wideband digital radio, a large memory size is required for each numerically controlled oscillator ( $\mathrm{NCO}$ ) and a large number of multiplications for each digital filter.

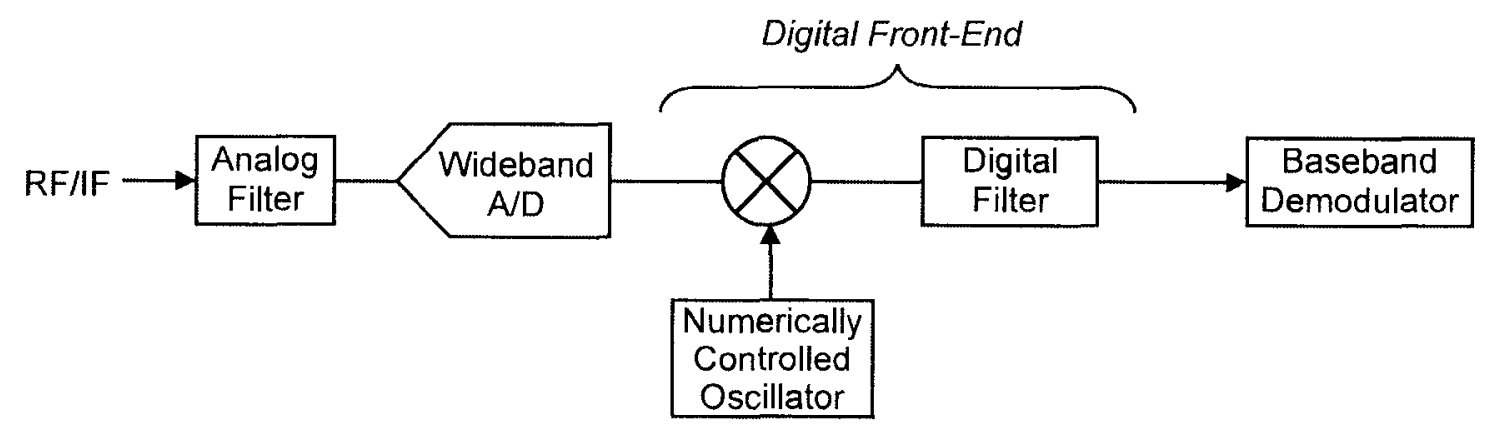

Fig. 1.2 A Digital Radio Using Wideband A/D and NCO.

The conventional approach to receiver design places a narrowband $\mathrm{A} / \mathrm{D}$ converter at baseband and leaves channel selection and filtering to an analog front-end, as illustrated in Figure 1.3. For a radio with only one channel selected, the wideband A/D shown in Figure 1.2, becomes a waste of a precious resource, because unselected channels, though useless, are still $\mathrm{A} / \mathrm{D}$ converted. One solution is the use of a bandpass sigma-delta 
modulation (SDM) based A/D converter, which can selectively convert a channel from a wide frequency bandwidth into the digital domain. This is shown in Figure 1.4.

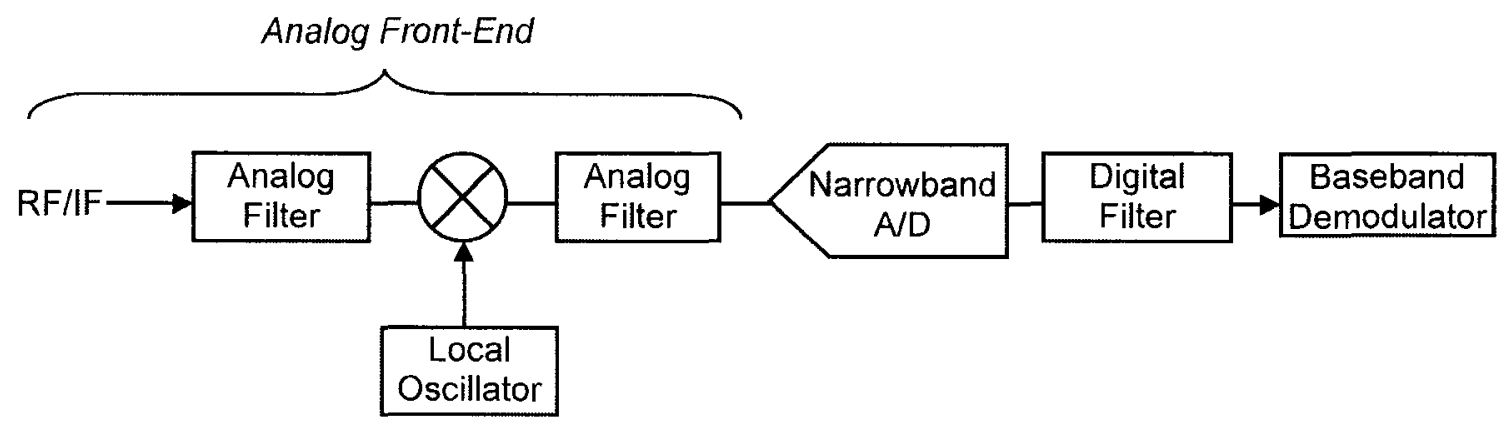

Fig. 1.3 A Radio with Analog Front-End and Narrowband A/D at Baseband.

Since first being introduced in a transmitting system in 1960 [1], sigma-delta modulation has been widely accepted as an efficient sampling technique for $\mathrm{A} / \mathrm{D}$ conversion. A sigma-delta modulator (SDM) samples a signal at a rate much higher than the Nyquist rate while using a smaller number of bits. The inevitable large quantization noise is suppressed to a minimum at the channel of interest. By doing this, the sigmadelta modulator achieves a high dynamic range at the selected channel with a smaller number of bits. Efficient $\mathrm{A} / \mathrm{D}$ conversion can then be accomplished by adding proper digital filtering stages following the SDM.

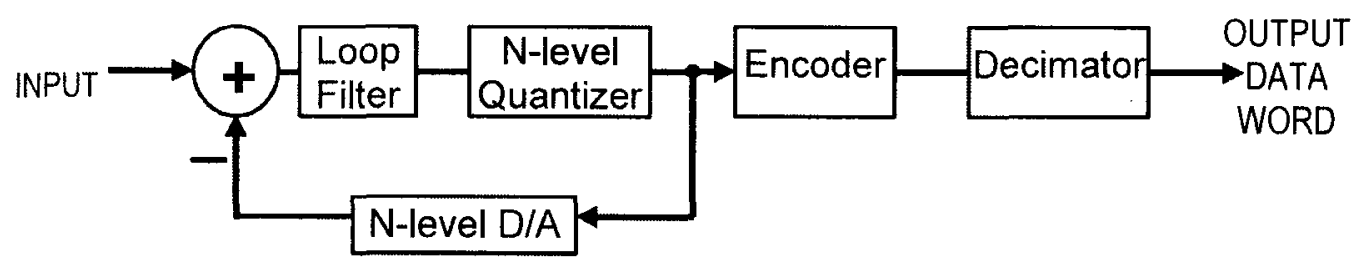

Fig. 1.4 Sigma-delta Modulator

The 1-bit sigma-delta modulator is easy to implement and absolutely linear in its feedback path. Its disadvantage is inherent instability in its high-order and full gain 
noise-shaping loop. Reducing the gain can make the 1-bit SDM more stable but at the expense of reduced bandwidth and increased design complexity. The bandwidth can in theory be expanded by increasing the oversampling ratio, but this comes with two kinds of problems. A high oversampling ratio and hence a high clock rate, requires high-speed circuitry, resulting in high power consumption and large clock feedthrough. Further, for a passband SDM, increasing the clock rate will shift the passband higher. Another approach is the multi-stage SDM (MASH) architecture that ensures overall stability by cascading multiple stable stages. However, it has mismatch problems among the stages.

Sigma-delta modulation is a trade-off between sampling rate and sampling bits. On one end of the spectrum is the 1-bit SDM, and on the other end the Nyquist-rate A/D converter, which employs a large number of bits and a low sampling rate. The large number of sampling bits requires high precision analog components, a limiting factor for the Nyquist-rate A/D. The multi-bit SDM is somewhat in the middle of the trade-off. Compared to a 1-bit SDM, the multi-bit SDM is more stable and capable of doing full gain noise-shaping. Dynamic range increases 6-dB for every added bit. In addition, the design of a multi-bit SDM can be much simpler than the 1-bit SDM. Since a single loop and a single stage structure can be implemented, the adjustment of loop delay becomes much easier.

A drawback with the multi-bit SDM is nonlinear element mismatch in the feedback D/A. In the multi-bit D/A converter, elements such as capacitors, resistors, or current sources are configured in such a way that a selected number of the elements release their electrical energy into a summing junction that produces an analog output signal equivalent to the digital input. Variation inevitably exists among the elements due to process and temperature variations. Although the absolute error from one element to another can be tightly controlled, the cumulative effect of the mismatched elements can cause significant nonlinear distortion. The element mismatch problem does not arise in the 1-bit SDM because perfect linearity is guaranteed by virtue of the fact that only a single element is used to convert the digital signal to analog.

Efforts to tackle the element mismatch problem include costly, precise laser trimming and calibration, and dynamic element matching (DEM). In DEM the D/A elements are selected in a way that their in-band noise is suppressed. However, the 
DEM adds complexity and time delay in the loop, and is susceptible to tonal problems, making high order SDM cumbersome.

\subsection{Motivation}

Though extensive research effort has been done on DEM to tackle the element mismatch problem in multibit SDM, there is a lack of quantitative understanding of the impact of the mismatch problem. This understanding needs to be achieved in comparison with a 1bit SDM that has no element mismatch, under reasonable levels of mismatch error and for typical communications systems. Insights can be gained through such practical studies. Also system noise of a reasonable level needs to be considered, because any improvement below system noise by the DEM is unnecessary.

Multibit sigma-delta modulators have received growing research interest recently [74]. One reason is the advent of new wireless applications such as 802.11 and 802.16 that demand more and more bandwidth. It is also possible that SDM can include some radio signal processing functions, and that can make the radio receiver more efficient. It was therefore the author's motivation to study multibit SDM in general, and bandpass multibit SDM in particular, for radio receiver applications.

\subsection{Objectives}

An objective of the present research was to study the reference feedback multibit sigmadelta modulator. Though previously placed in a SDM circuit [69], it has not been investigated in any detail. We have explored the potential advantages of this type of SDM in terms of noise-shaping performance and radio signal processing.

Another objective was to conduct a quantitative comparison of the multibit SDM vs a 1bit SDM regarding the impact of D/A element mismatch error, in typical radio 
applications. This helps to make design choices and trade-offs in engineering multibit SDM.

A further objective in this research was to look for some efficient circuitry in continuoustime (CT) SDM. As a particular form of SDM, the CTSDM offers advantages in speed, noise, and power consumption, and hence is a potential contributor in next generation radio technology.

\subsection{Contributions}

The reference feedback multibit sigma-delta modulator was studied in-depth for the first time, to the author's knowledge. The study shows that the reference feedback SDM offers a signal-matched noise-shaping loop over process and temperature variations, which is advantageous to reference circuit design and dynamic range \& AGC control. A fourth-order CT bandpass reference feedback SDM was designed and built (Chapter 6); experiments was conducted in comparison with a conventional SDM that has a summingtype DAC in the feedback path (Chapter 7).

A novel biquad circuit was developed for the continuous-time SDM loop filter. By cascading the novel biquad with regular biquad circuits, any higher order loop filter can be easily designed and realized (Section 6.2). The novel biquad was successfully implemented and tested in the $4^{\text {th }}$-order CT loop filter design.

The bandwidth advantage of a full-gain multibit SDM compared with a reduced-gain 1bit SDM was identified for the first time, to the author's knowledge (Figure 5.7).

A quantitative comparison of the multibit vs the 1-bit SDMs was conducted in terms of dynamic range for the first time, to the author's knowledge. It shows that with a moderate level of D/A element mismatch, a multibit SDM without the use of DEM is 
better than a 1-bit SDM for bandwidths above $500 \mathrm{kHz}$, suggesting that the multi-bit SDM is a better choice for broadband applications (Section 5.5).

An insightful discussion on the stability and linearity of the SDM nonlinear feedback noise-shaping loop is provided (Section 5.2).

A new clock structural model for a rational factor, sampling-rate-conversion digital filter is provided (Section 4.2.3). The new model can be a helpful tool in designing efficient sampling-rate-conversion digital filters.

A unified, alias-free sampling condition for both lowpass and bandpass sampling is derived (Equation 3.12 in Section 3.3), which is of some theoretical significance. A more general and simple alias-free condition for quadrature sampling is also provided in (3.14), (3.15a) and (3.15b).

Two papers have been published based on this research work [75][76]. 


\section{CHAPTER 2 Radio Receiver Review}

Integration and digitization are two major trends in the advancement of radio technology. It started in the late 1970 s when silicon bipolar ICs were first introduced in the IF and baseband of radio receivers. Integration did afford the freedom to use transistor-rich circuits for higher performance. Circuit techniques such as double-balanced mixers using Gilbert analog multipliers, PLLs as FM demodulators, and the use of balanced on-chip signal paths became widely used. By eliminating many of the coupling coils and other non-critical discrete components found in older circuits, ICs contributed to lowering the cost of assembling and aligning of the final product [16]. Receiver size and power dissipation has been reduced drastically. Two chip solutions, one for RF/IF and another for baseband, are common practice today. Single-chip radios that integrate all functions onto one chip represent the highest level of integration.

The digitization trend in radio is to perform as many functions as possible using digital circuits and their accompanying software. The most aggressive approach is the so-called software radio [4][23][24][25][26], in which the whole wideband signal at $\mathrm{RF} / \mathrm{IF}$ is digitized and then processed by a general-purpose digital signal processor. Since the general-purpose digital signal processor is programmable, highly flexible radios can be built. However, most radios only receive one channel at a time, and it is highly wasteful to sample the whole band when only one channel is needed. Current efforts are focused on software-defined radios [23][24][25][26], in which only a certain bandwidth at a relatively low IF is sampled to cover two or more radio standards. 


\subsection{Receiver Basics}

In the process of extracting digital bits from a modulated sinusoid waveform, a radio receiver performs two basic functions: channel selection and waveform demodulation. A functional block diagram for a radio receiver is shown in Figure 2.1. The lines between the functional blocks are not sharply drawn. For example, channel-select filtering and carrier recovery can also be performed in the baseband demodulator. Some functions are distributed among stages. However, the channel-select function is usually performed by the front-end, and waveform demodulation by the baseband demodulator.

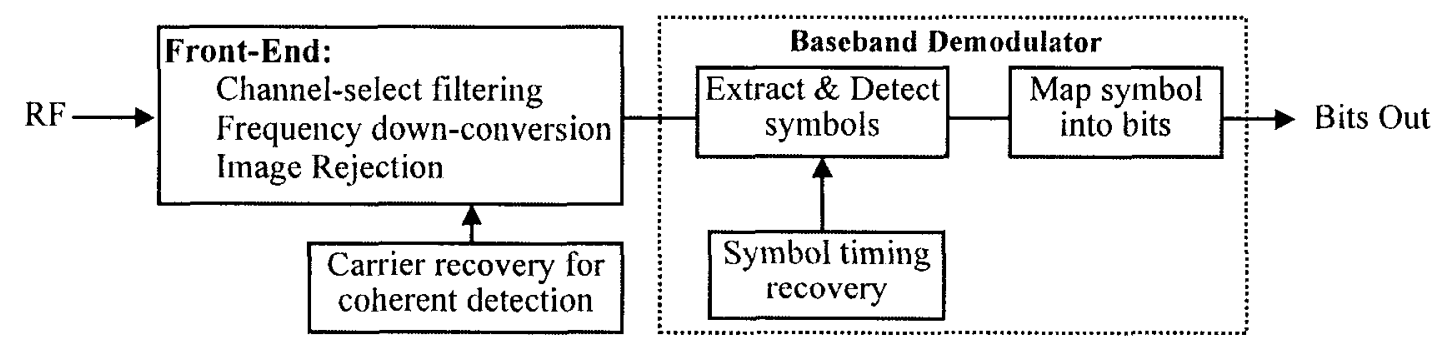

Fig. 2.1 A Functional Block Diagram of Digital Radio Receiver.

The front-end block of a receiver remains mostly analog whereas the baseband demodulator normally is digital; $\mathrm{A} / \mathrm{D}$ conversion taking place between the two functional blocks. Further digitization of the radio would require the $\mathrm{A} / \mathrm{D}$ to be moved into the front-end, allowing more signal processing to be performed digitally. The following review will focus primarily on the front-end.

\subsubsection{Channel Selection}

The received spectrum is normally wideband with multiple channels. Channel selection is necessary to choose the desired signal while rejecting all others. This is commonly done by means of filtering. At the same time, the signal frequency is down-converted from RF to IF or even baseband, leaving a narrowband and slowly-changing signal for 
demodulation. Since each channel has a different carrier frequency, the down-conversion must be tunable so that any one of the channels can be selected. The frequency channel tuning is normally performed through a frequency synthesizer based PLL, known as a local oscillator (LO). Tunable LO mixing with the selected signal channel yields an output at a fixed center frequency, leading to the use of filters with fixed passband for channel-select filtering.

The filtering is determined by the power profile amongst the channels, the worst case would be when adjacent channel signals reach their maximum while the desired signal is at its minimum. High-Q filters are the best in performance but are bulky. They are often found in conventional radios. An approach for a high level of integration is to distribute the channel filtering amongst analog and digital stages where the use of on-chip low-Q filters is feasible.

\subsubsection{Image Rejection}

A critical signal processing function during the frequency down-conversion is image rejection. The image frequency is twice the LO frequency minus the selected channel frequency. Such an image, if present, will be right on top of the desired signal channel after mixing, making filtering impossible. There are basically three strategies for image rejection: pre-filtering (before mixing), post-cancelling (after mixing), and directconversion (zero-IF). The pre-filtering employs filters before the mixer as illustrated in Figure 2.2. It is usually found in conventional analog radios with bulky high-Q filters to achieve good image rejection.
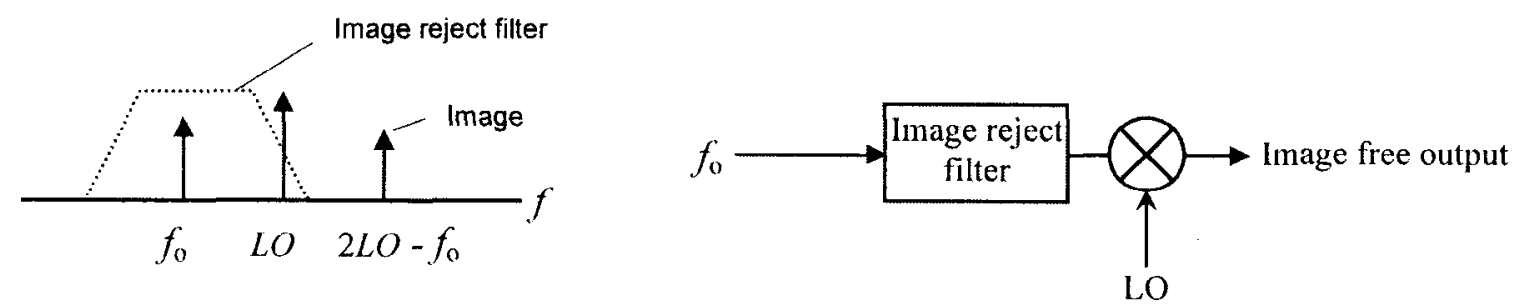

Fig. 2.2 Image Rejection by Pre-filtering Before Mixing 
The post-cancelling strategy, widely known as image-reject mixing, uses two identical mixers to down-convert the signal into in-phase and quadrature (I/Q) signal components, and having one of them undergo a 90-degree phase change (Hilbert transform) before combining, as shown in Figure 2.3. The output will be image free. The post-cancelling strategy is suitable for IC integration because good amplitude and phase balance between the two signal paths can be obtained. For example, $20 \mathrm{~dB}$ image rejection (corresponding to about ten degree phase imbalance) can be normally achieved off-chip [44], whereas 40 $\mathrm{dB}$ (corresponding to about one degree phase imbalance) can be achieved only with significant difficulty on an IC [45].

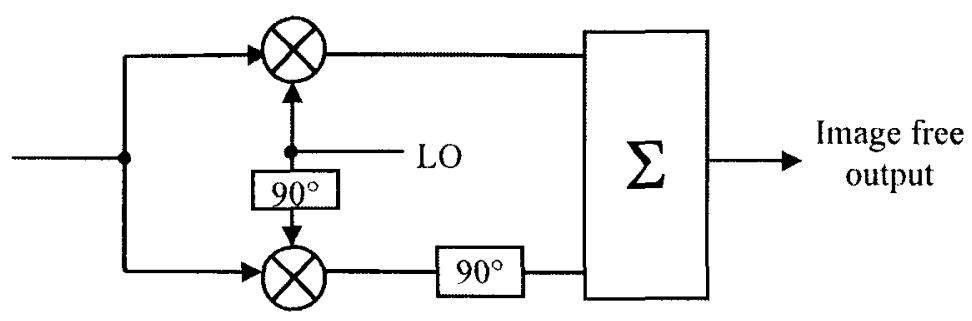

Fig. 2.3 Image Post-Cancelling After Mixing

With the direct conversion or zero-IF strategy, there is no image to reject, or to say the same thing in a different way, the image is the signal itself. This happens when the LO frequency equals the signal carrier frequency. In this case, the components associated with the image rejection processing are eliminated; a very attractive feature for high level integration such as for a single-chip radio. However, one drawback associated direct conversion is the lack of signal isolation between the LO port and the signal path (as is present in super-heterodyne radios), resulting in a dynamic dc offset problem. More details will be given in the section 2.2.3.

\subsubsection{Dynamic Range}

Dynamic range defines the range of a signal level for which a receiver will work. At the low level is the minimum detectable signal (sensitivity) above the noise floor, which is 
determined by thermal noise plus added noise from within the receiver. For a particular signal format and modulation $\&$ demodulation scheme, the minimum detectable signal is a fixed number relative to the noise floor. The receiver-added noise often is much higher than the thermal noise. It is therefore critical to keep the receiver-added noise under an acceptable level. An important noise source is the phase noise from the LO. The effect of phase noise is two-fold. First, when the LO is mixed with the desired channel, the phase noise will contribute directly to the total noise. The second effect occurs when the LO is also mixed with strong adjacent channel signals (reciprocal mixing). The phase noise sideband of the strong adjacent channel signal can be significant and will add to the total noise in the desired channel.

At high signal levels, the dynamic range is limited by non-linearity. Non-linear operation creates new frequency components causing signal distortion. The distortion becomes stronger as the signal level increases; at some point the receiver will not be able to function properly. A well-known nonlinear product is the third-order intermodulation $\left(2 f_{2}-f_{1}\right)$. When $f_{1}=f_{0}+2 \Delta$ and $f_{2}=f_{0}+\Delta$, where $f_{0}$ is the channel of interest, $f_{1}$ and $f_{2}$ are the adjacent channels, and $\Delta$ is the channel spacing, the third-order intermodulation of the adjacent channels will be $\left(2 f_{2}-f_{1}\right)=f_{0}$, right on top of the channel of interest, making filtering impossible.

The dynamic range can be increased by adding AGC. The extension is usually for high signal levels and the benefit can be huge for post-AGC stages such as the A/D and digital filters because a smaller number of bits is required. Not surprisingly, the expense is at the low signal level due to AGC-added noise.

In receiver design and implementation, there are many architectural choices for performing the same function, yet constraints from the manufacturing process and economical concerns add an extra degree of complexity. In the following sections, we will briefly review some typical receiver architectures, by way of examples. The review is arranged from conventional analog to highly digital, with a high level of integration. 


\subsection{Analog Receivers}

Receivers having $\mathrm{A} / \mathrm{D}$ converters at their baseband perform the channel-select function by analog front-ends. This includes image rejection. channel-select filtering, channel tuning, and AGC. The final lowpass filtering for the channel-select can also be performed in part by the digital baseband.

\subsubsection{Conventional Super-Heterodyne}

This type of receiver is typically of a low level of integration, with many discrete components. An example of a dual-conversion super-heterodyne with a single IF is illustrated in Figure 2.4. The variable LO in the first conversion stage performs channel tuning and produces an output at a fixed intermediate frequency (IF). The IF is at least half of the bandwidth so that the out-of-band image can be rejected by a RF bandpass filter prior to the mixer. The higher the IF, the lower the $Q$ of the filter.

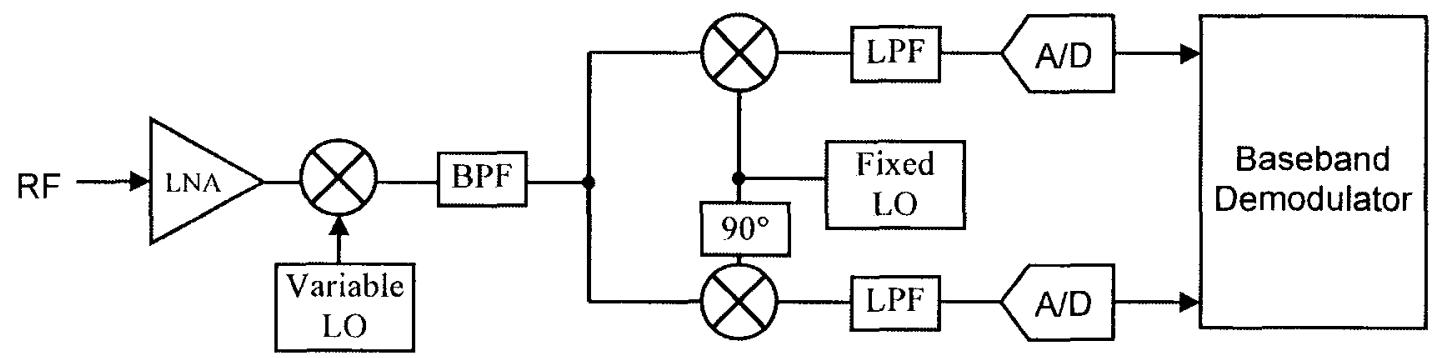

Fig. 2.4 A Dual-Conversion Super-Heterodyne Radio Receiver

The fixed LO is at the same frequency as the IF thus bringing the selected signal channel down to baseband. The channel-select filtering is distributed between the bandpass filter at IF and the lowpass filters (LPF) at baseband. Depending on blocking performance requirement, a bulky high-Q SAW filter is usually needed. Also high-Q resonators are 
needed to ensure lower phase noise in LOs. Though difficult for integration, the high-Q components provide excellent channel selectivity, signal sensitivity, and linearity for the super-heterodyne radio.

The second stage in Figure 2.4 converts the selected channel to zero-IF or baseband. If implemented on-chip, the subsequent lowpass filters (LPF) will be low-Q and may not be good enough to suppress adjacent channels. The choices will be to either use a higher-Q BPF at IF, or to increase the dynamic range of the A/D converters and remove the blockers digitally in the baseband. Both can be expensive choices. If the second stage is implemented off-chip, two high-Q LPFs can be used. However, the phase and amplitude balance will not be as good as for an on-chip solution. One alternative approach is to have two or more IF stages, thus easing the filtering requirements for each stage. Generally, for a given technology, a certain amount of signal-processing power is needed to achieve the desired receiving performance in terms of signal-to-noise (SNR) or bit error rate (BER). This processing power can be distributed among the stages. Tradeoffs between analog and digital can always be made depending on the particular application.

\subsubsection{Integrated Super-Heterodyne}

The super-heterodyne can be highly integrated by use of image reject mixing. The image suppression will rely on the I/Q phase and amplitude balance. An example wideband IF receiver is shown in Figure 2.5 [43], in which all the functional blocks can be implemented on-chip without any high-Q discrete bandpass filters.

The first fixed LO helps to lower phase noise because a small frequency-divide ratio and a large loop bandwidth can be used for the PLL circuit. In this case, the IF must be large enough for the whole signal band to be captured and down-converted to IF. The subsequent LPFs are low-Q whose function is just to remove the sum frequency components. The second stage selects and directly down-converts a channel of interest to baseband with the variable LO. In terms of phase noise performance, varying the LO at 
IF is better than at RF (as in a conventional super-heterodyne), but the LO tuning range will be reduced. In Figure 2.5 there are two sets of image reject mixers intertwined to provide a complex baseband output to the $\mathrm{A} / \mathrm{D}$, achieving the image rejection through quadrature down-conversion instead of through a 90-degree phase shifter. Channel select filtering can be distributed between the on-chip LPFs prior to the $A / D$ and the digital filters in the baseband demodulator.

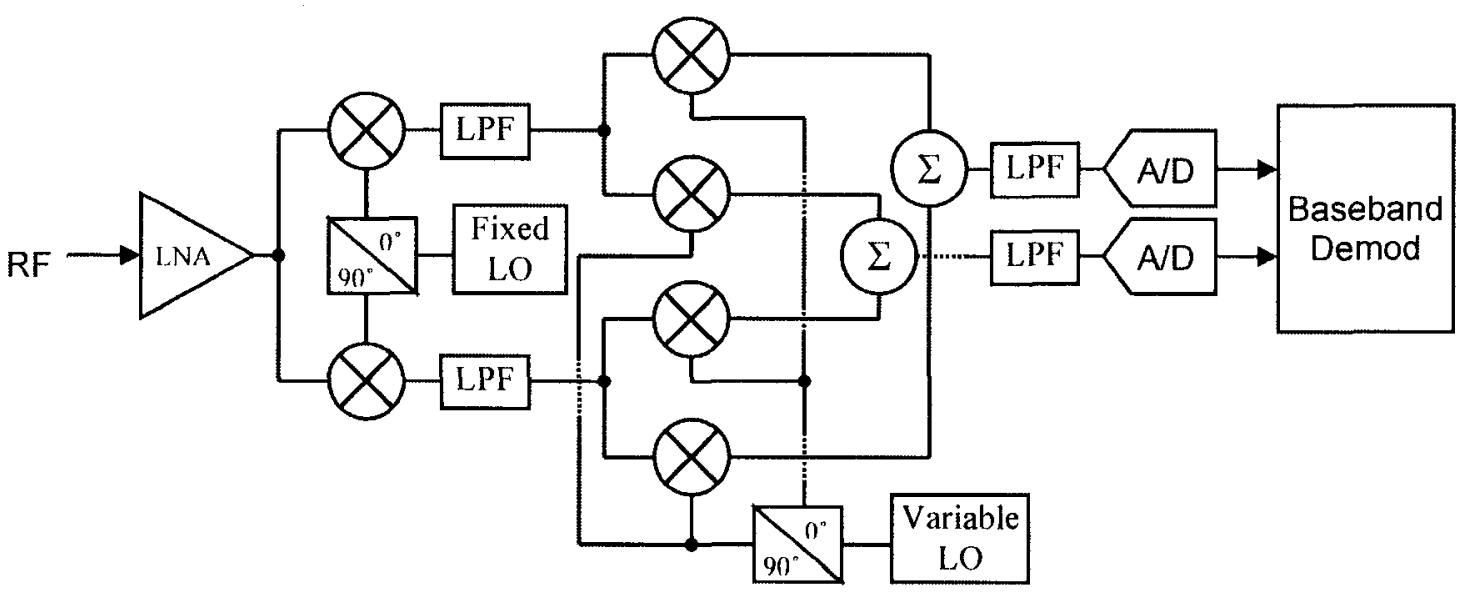

Fig. 2.5 A Highly Integrated Super-Heterodyne Radio Receiver.

Drawbacks to this architecture include increased power consumption, and added noise and nonlinearity from the additional mixers. The channel tuning range is expected to be lower due to the variable LO being implemented at IF. Image rejection is further limited by gain \& phase matching among all four signal paths at the second stage. Since it is a direct-conversion at the second stage, there are also DC offset and drift problems [43][44]. However the dual conversion super-heterodyne has no problem arising from carrier feedthrough as does a direct-conversion radio.

\subsubsection{Analog Direct-Conversion}

It is very attractive to bring the RF signals down directly to baseband or zero-IF. Also referred to as a homodyne, this architecture was proposed as early as 1924 for crude 
receivers requiring only a single vacuum tube. The direct-conversion radio was actually implemented in a telephone measurement instrument in 1947.

Since the desired channel is directly down-converted to zero-IF, there is no image frequency. Thus components associated with image rejection processing are eliminated, reducing the number of components significantly. This is very attractive for single-chip radios, since channel selection can be done by an on-chip lowpass filter. The mixer in a direct-conversion receiver must be highly linear to suppress unwanted inter-modulation products from interferers. An example of a direct conversion radio receiver is shown in Figure 2.6.

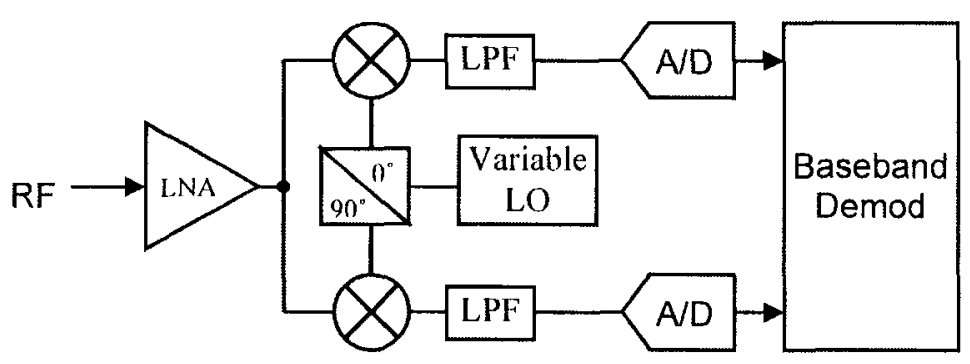

Fig. 2.6 A Direct-Conversion Radio

A serious problem associated with direct-conversion is time-varying dc offset, arising from poor isolation between the RF signal and the LO since they operate at the same frequency. LO leakage to the antenna can be partly reflected back and mixed with the LO itself, creating a dc component. This dc component is time-varying because the reflection is dependent on the antenna impedance matching, which is sensitive to its environment. In addition, a large near-channel interferer can leak into the LO port of the mixer and self down-convert to dc. Good circuit design may reduce these effects to a certain extent, but cannot totally eliminate them [16]. Attempts have been made to notch out the DC from the baseband signal. This method performs well only if the signal contains little or no information at or near DC. Moreover, flicker (1/f) noise in the mixers and the amplifiers, which degrades the SNR or reduces the sensitivity of the receiver, also becomes a concern. 


\subsection{Digital IF Receivers}

Since the challenge for digitizing at RF is still enormous with current technology, digital IF receivers are an appropriate choice. This implies an analog RF front-end prior to the digital IF receiver. A critical choice is whether to perform the channel tuning at RF or IF. Digitizing IF is advantageous, because IF analog filtering and down conversion, which generally have poor phase performance and tend to introduce inter-symbol interference, can be avoided. Digital filtering and down conversion are free from these problems. Also, an A/D converter with enough dynamic range can eliminate the analog $\mathrm{AGC}$ and improve adjacent channel rejection and overall SNR. Furthermore, there is no flicker $(1 / f)$ noise issue in the digital IF receiver, as seen in an analog direct-conversion receiver, and perfect I/Q amplitude and phase balance can be easily realized digitally.

\subsubsection{Wideband Digital IF Receiver}

This architecture, as shown in Figure 2.7, is simply a digital version of an analog IF direct-conversion receiver. The analog mixer is replaced with a digital multiplier, and the analog local oscillator with a numerically controlled oscillator (NCO). The NCO generates cosine and sine numbers to multiply with the digitized wideband signal; the selected channel is down converted to a complex baseband signal, which is decimated and filtered digitally. Lowpass decimation filters are usually implemented by efficient cascaded-integrator-comb (CIC) filters, then low rate channel filtering can be done by standard digital filters.

The NCO performs channel tuning. Usually implemented with a look-up table (LUT), the NCO takes a lot of registers or memory size when a large number of bits (high dynamic range) and a small tuning step are required. In place of phase noise, the level of spurs is of concern for an NCO due to the finite number of output frequencies [53]. Spurs are created from regular phase error accumulation when the output 
frequency is not an integer number of $\mathrm{Fs} / 2^{\mathrm{N}}$, where Fs is clock frequency and $\mathrm{N}$ is the number of bits. Dither techniques are often employed to randomize the regular phase error accunulation process, spreading the spurs into flat noise.

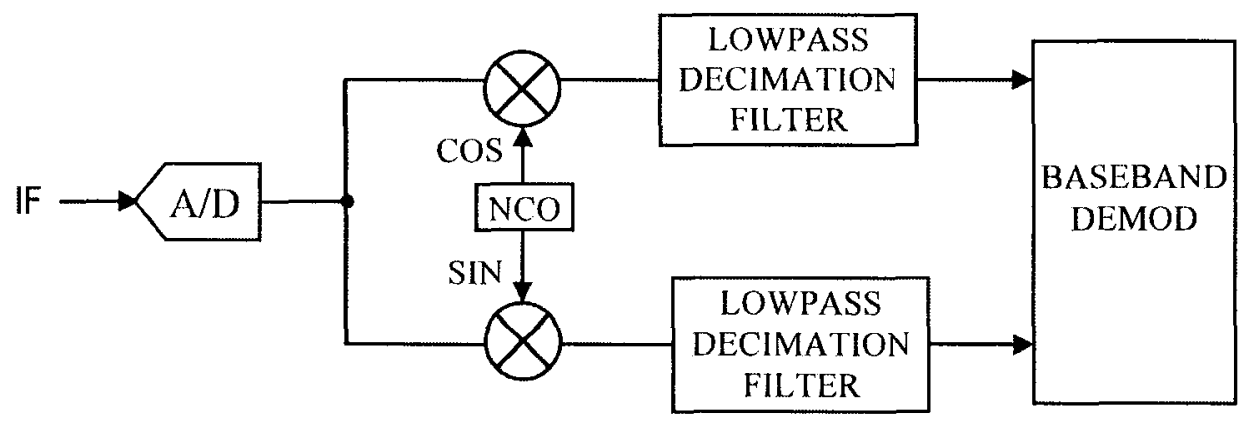

Fig. 2.7 Standard Digital IF Receiver

For receivers that select only one channel (as most radios do), the wideband digital IF receiver is wasteful of resources, since all the digitized channels except the desired will be thrown away by digital filtering. Yet, extra dynamic range, or number of bits, is put in place for them. It would be better to remove them earlier and save precious resources. This can be realized with bandpass sigma-delta modulation, as will be discussed later.

A variation of the wideband digital IF architecture is the time-varying filter [31]. Time-varying filters often are seen in multi-rate (sampling rate conversion) digital filters [35][36]. The approach is to pre-compute the multiplication of the LPF coefficients with the NCO output. This actually is re-arranging the multiplication order amongst the input data. NCO output, and filter coefficients as follows.

$[($ Input Data $) \times(\mathrm{NCO})] \times($ Filter Coeff $)=($ Input Data $) \times[(\mathrm{NCO}) \times($ Filter Coeff $)]$

The pre-computed data are stored in memory. Since the NCO output data changes every sample, it is equivalent to a change of filter coefficients, known as a time-varying filter. 
Also, because the NCO data is periodic, the number of pre-computed coefficients is limited, and therefore can be stored. The result is a reduction in real-time multiplication. This amounts to an exchange of memory size for speed. However, an increase in memory size can be costly in radio applications where many channels need to be covered.

\subsubsection{Narrowband Digital Low-IF Receiver}

To avoid the DC offset and flicker (1/f) noise problems that trouble analog direct conversion architecture, and yet still achieve the high level of integration without wasted resource, narrowband digital low-IF architectures can be considered. Post-cancellation for image reject is implemented at a low-IF, by analog or by digital means. For instance, analog complex filtering, as shown in Figure 2.8 [47], can do the image rejection before the $A / D$, easing the dynamic range requirement on the $A / D$ and subsequent digital stages. The disadvantage of this is the imperfect performance of analog complex filtering at lowIF.

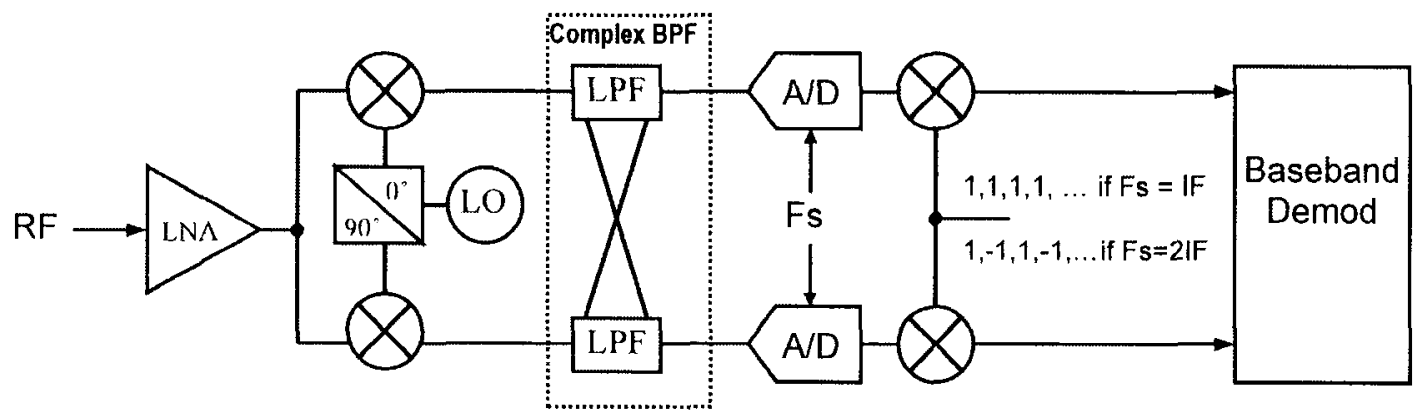

Fig. 2.8 A Low-IF Receiver with Complex Filter Prior to A/D Conversion.

Digital post-cancelling of the image, on the other hand, requires $A / D$ converters with larger dynamic range. One example of such an architecture is shown in Figure 2.9. It is the same configuration as in Figure 2.5 for the integrated super-heterodyne receiver, but realized in digital form. Its advantage is the use of real pre-A/D filters. The frequency independent amplitude error and imperfect image-rejection from the real filters can be 
corrected for by means of a simple AGC algorithm in the DSP [47]. Excellent digital I/Q amplitude and phase balance are expected.

In both cases, it is beneficial to have the IF to be a quarter of the sampling frequency, or an integer multiple of the sampling frequency, known as quadrature sampling or integer sampling, respectively, so that digital mixing can be implemented efficiently.

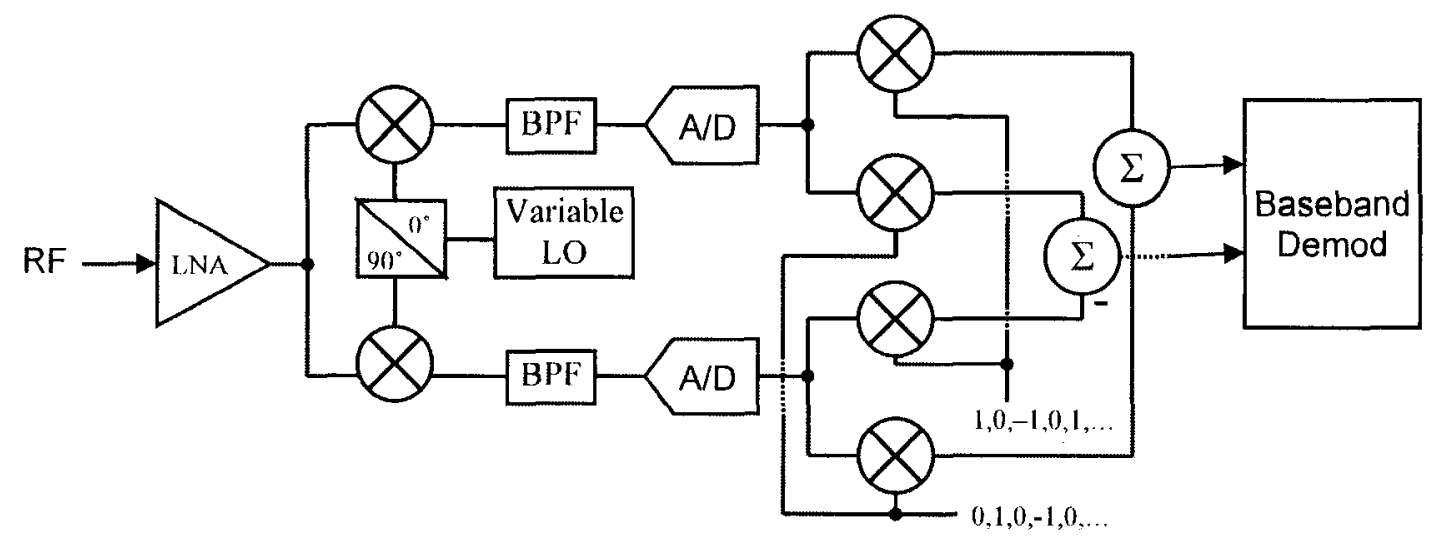

Fig. 2.9 A Low-IF Receiver with A/D Converters Before Image Rejection.

Another example known as a software-defined radio (SDR), is shown in Figure 2.10. Here a quadrature sampling architecture has been implemented [23][24][25][26]. It operates on two radio standards, a $200 \mathrm{kHz}$ channel bandwidth for the DCS1800 standard, and a $1.6 \mathrm{MHz}$ channel bandwidth for UMTS frames.

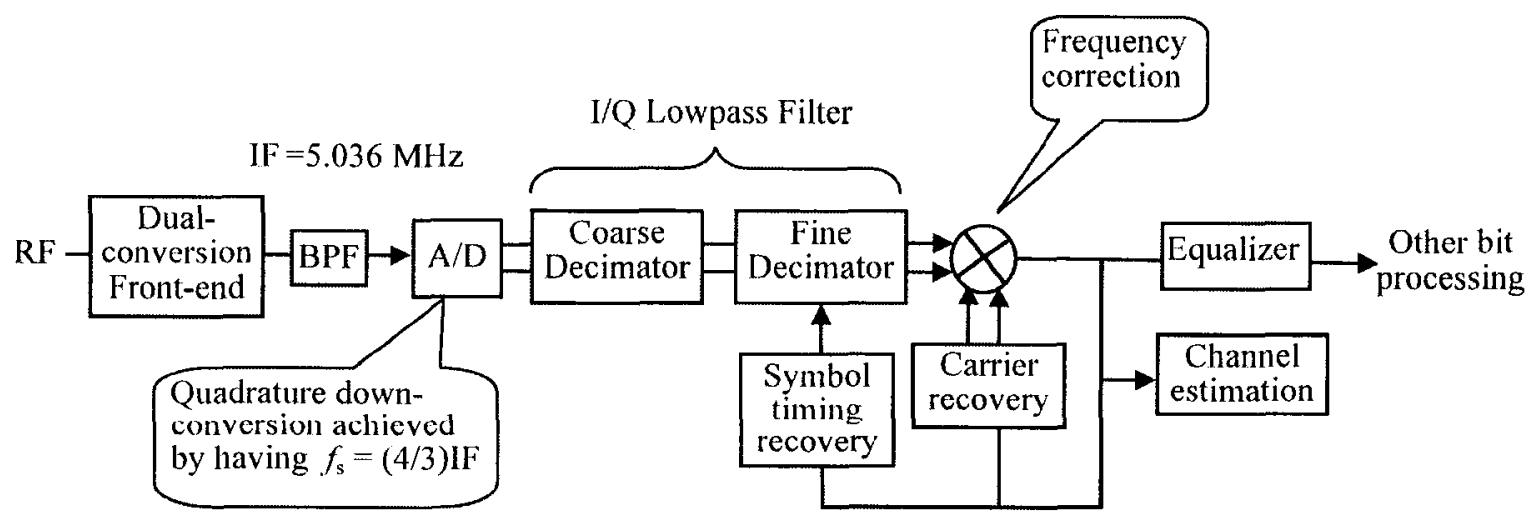

Fig. 2.10 A Quadrature Sampling Software-Defined Radio Architecture 
An analog dual-conversion RF stage is in the front-end and performs the channel tuning and image rejection functions. Quadrature sampling of any channel is accomplished by fixing the IF at $5.036 \mathrm{MHz}$ and $\mathrm{A} / \mathrm{D}$ sampling rate at four thirds of the IF [23]. The computational load for channel filtering, which dominates the total computational complexity, is reported as 30 million MACS for a 4-pole Chebychev IIR filter or 84 million MACS for a 200-tap FIR decimation filter with a decimation factor of 8 [24].

\subsubsection{Sub-Sampling Receiver}

In super-heterodyne and homodyne architectures, frequency down-conversion is performed by either switch- or multiplier-based mixers. The switching action performed in the switch-based mixer is similar to that in sampling. It is therefore no surprise that digital sampling can be employed for frequency down conversion. Such techniques will be reviewed in general in the next chapter. What is reviewed here is a particular subsampling receiver that down-samples the RF signal to a low-IF.

A sub-sampling receiver, without the use of external IF filters, is illustrated in Figure 2.11 [12]. The sampling frequency is chosen in such a way that the IF frequency after the sub-sampling will be one third of the sampling frequency. Assuming an RF of $910 \mathrm{MHz}$, the sampling clock frequency would be $78 \mathrm{MHz}$, which down-converts the 910-MHz to $26 \mathrm{MHz}$. This is also called bandpass sampling and will be reviewed in the next chapter. Subsequent decimating stages are done by factors of 2 , so that after folding, the IF signal frequency is still one third of its sampling rate. The advantage of doing this is that the same switched-capacitor anti-aliasing filter can be implemented [12], and the discrete-time signal processing can avoid some of the quantization errors normally seen in a purely digital approach.

It is not clear in paper [12] how to perform channel tuning, since there is a fixed ratio between the RF and the sampling clock, which is generated from a high-Q resonator and is not variable to the degree necessary for channel-tuning purposes. In addition, a 
costly NCO may be needed in the subsequent digital signal processor to convert the lowIF signal to baseband.

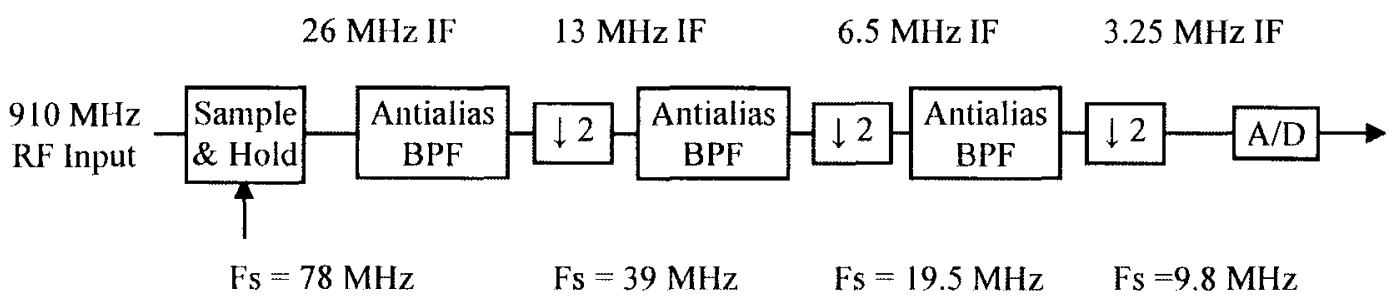

Fig. 2.11 A Sub-Sampling Radio [12]

Moreover, since the signal is down-converted from RF, sub-sampling acquires wideband noise and aliases the noise into a bandwidth at half the sampling rate. This raises the baseband noise spectral density by the ratio of the track bandwidth to the sampling rate. Bandpass sampling therefore has a higher noise figure than the analog mixer scheme. However, since the process tends to be more linear than an analog mixer, its dynamic range is also large, unless a high gain pre-amplifier is employed [16].

\subsubsection{Bandpass Oversampling Receiver}

Noise-shaped oversampling or sigma-delta modulation (SDM) has been studied extensively [1][3]. It samples a signal at a rate much higher than the Nyquist rate while only using one or a few bits. In-band quantization noise is deliberately suppressed at the expense of increased out-of-band noise. By doing so, the sigma-delta modulator achieves a high dynamic range with a smaller number of bits. Such techniques have been successfully applied to lowpass AVD converters [1][3][41].

For radio applications, it is advantageous to have bandpass type sigma-delta modulation. In contrast to the Nyquist-rate sampling, noise-shaped oversampling on only the channel of interest will save precious hardware resources by using a few bits, without 
sacrificing dynamic range. Further, compared to lowpass SDM, the bandpass SDM can avoid DC offset and 1/f noise problems seen in the direct-conversion radio by directly sampling the IF signal. The bandpass SDM was first proposed in 1988 to extend the lowpass sigma-delta modulation to a bandpass one [32]. The feasibility of bandpass sigma-delta modulation has been extensively studied and demonstrated [34][33][41][42]. More specifics will be discussed in the Chapters 5 and 6 .

\subsection{Summary}

Radio receivers are briefly reviewed in this chapter. Interference and crowded channels make radio design a very challenging task, requiring precise frequency conversion and a large amount of filtering. The migration to highly integrated digital radios just makes it even more challenging. In a digital radio, SDM or oversampling offers an efficient solution for $\mathrm{A} / \mathrm{D}$ conversion, since only the desired channel is captured. In the following table, the digital receiver architectures reviewed in this chapter are summarized in terms of level of integration and digitization.

Table 2.1 Comparison amongst the Receiver Architectures

\begin{tabular}{|c|c|c|c|c|c|}
\hline $\begin{array}{c}\text { Digital Receiver } \\
\text { Architecture }\end{array}$ & $\begin{array}{c}\text { Digitization } \\
\text { Level }\end{array}$ & $\begin{array}{c}\text { Integration } \\
\text { Level }\end{array}$ & Complexity & Cost & Performance \\
\hline $\begin{array}{c}\text { Conventional } \\
\text { Super-heterodyne }\end{array}$ & Low & Low & Low & High & Excellent \\
\hline Wideband IF & Low & High & High & Medium & Medium \\
\hline Direct Conversion & Low & High & High & Low & Poor \\
\hline $\begin{array}{c}\text { Standard } \\
\text { Digital IF }\end{array}$ & High & High & Medium & High & Good \\
\hline Low-IF & Medium & High & High/Medium & Low & Good \\
\hline Sub-sampling & Medium & High & High & Low & $?$ \\
\hline $\begin{array}{c}\text { Bandpass } \\
\text { Over-sampling }\end{array}$ & Medium & High & High & Very Low & $?$ \\
\hline
\end{tabular}




\section{CHAPTER 3 Sampling and Quantization}

Analog-to-digital conversion involves two types of operation: sampling and quantization. Sampling makes a signal discrete in time; quantization makes a signal discrete in amplitude. While sampling is a linear operation with no loss of information when performed properly, quantization is always nonlinear with added distortion, error, or socalled quantization noise. Quantization forces the signal output to some prescribed amplitude level, so that the signal can be represented or coded by digital bits. Between the sampling and quantization operations, discrete signal processing can be performed, but not bit by bit. It is a sampled-data operation, but not yet a digital one. For example, switched-capacitor filtering is a discrete-time and linear operation.

\subsection{Ideal and Natural Sampling}

Let $s(t)$ be the ideally sampled version of a signal $g(t)$ as illustrated in Figure 3.1. We have:

$$
s(t)=g(t) \sum_{n=-\infty}^{\infty} \delta(t-n T)=\sum_{n=-\infty}^{\infty} g(n T) \delta(t-n T)
$$

where $T$ is the sampling interval and $\delta($.$) is the well known Delta function.$

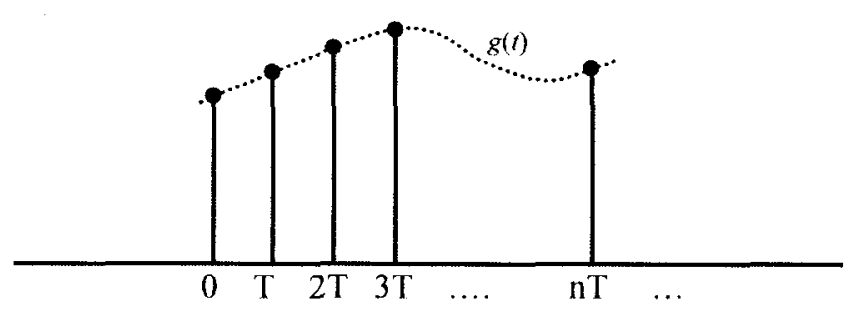

Fig. 3.1 Ideal Sampling 
The Fourier transform of $\delta(t-n T)$ is $\exp (-\mathrm{j} 2 \pi \mathrm{n} f T)$. Therefore, the Fourier transform $\mathrm{S}(f)$ of the ideally sampled signal $s(t)$ can be written as:

$$
S(f)=\sum_{n=-\infty}^{\infty} g(n T) e^{-j 2 \pi n f T}
$$

Another more useful expression can be obtained as follows. The Fourier transform of an impulse train is given by [10]:

$$
\text { FOURIER }\left\{\sum_{n=-\infty}^{\infty} \delta(t-n T)\right\}=f_{s} \sum_{n=-\infty}^{\infty} \delta\left(f-n f_{s}\right), \quad f_{s}=1 / T
$$

Using the convolution property of a Fourier transform on Eq. (3.1), the Fourier transform $\mathrm{S}(f)$ of the ideally sampled signal $\mathrm{s}(\mathrm{t})$ is given by

$$
S(f)=G(f) *\left\{f_{s} \sum_{n=-\infty}^{\infty} \delta\left(f-n f_{s}\right)\right\}=f_{s} \sum_{n=-\infty}^{\infty} G\left(f-n f_{s}\right)
$$

where $G(f)$ is the Fourier transform of $g(t)$ and $*$ denotes convolution. Thus, the sampling has produced images of $\mathrm{G}(f)$ at multiples of the sampling rate $f_{\mathrm{s}}$ on the frequency axis. If the images of $\mathrm{G}(f)$ overlap, aliasing occurs and the signal information is distorted and cannot be fully recovered. If the sampling rate $f_{\mathrm{s}}$ is equal to or greater than twice the highest frequency component in $\mathrm{G}(f)$ (Nyquist rate), overlap is avoided, and the original signal can be fully reconstructed. This is called the Sampling Theorem. The reconstruction formula, known as the Nyquist-Shannon interpolation formula, is as follows:

$$
g(t)=\sum_{n=-\infty}^{\infty} g(n T) \frac{\sin \left[\pi f_{s}(t-n T)\right]}{\pi f_{s}(t-n T)}
$$


The ideal sampling cannot be done in practice because it requires infinite bandwidth to implement the Delta function.

Another method of sampling is natural sampling, as shown Figure 3.2 where the signal is tracked upon sampling. The sampled signal $s(t)$ can be expressed as:

$$
s(t)=\sum_{n=-\infty}^{\infty} g(t) p(t-n T)
$$

where

$$
p(t-n T)= \begin{cases}1 & n T \leq t<n T+\tau \\ 0 & \text { otherwise }\end{cases}
$$

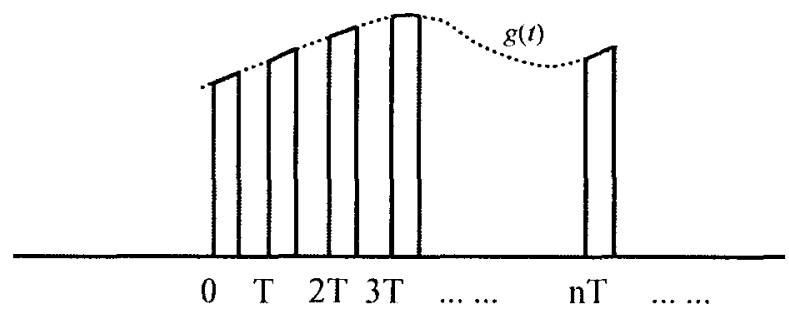

Fig. 3.2 Natural Sampling

A natural sampled signal can be recovered if the sampling rate $\left(f_{s}=1 / T\right)$ is equal to or greater than the Nyquist rate. However, when it comes to quantization, natural sampling does not offer any advantage over simple flat-top sampling, as will be shown in the next section.

\subsection{Flat-top Sampling and the Aperture Effect}

A widely used sampling scheme in practice is flat-top sampling. As shown in Figure 3.3, the sampled signal is held for a short interval. The sampled version is expressed as: 


$$
s(t)=\sum_{n=-\infty}^{\infty} g(n T) p(t-n T)
$$

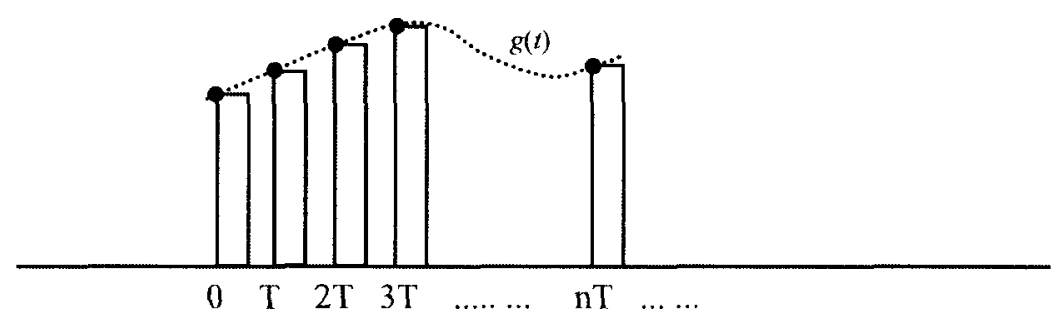

Fig. 3.3 Flat-Top Sampling

Using the convolution property of the Delta function $p(t)^{*} \delta\left(t-t_{n}\right)=p\left(t-t_{n}\right)$, Eq. (3.8) can be rewritten as:

$$
\begin{aligned}
s(t) & =\sum_{n=-\infty}^{\infty} g(n T)\{p(t) * \delta(t-n T)\} \\
& =p(t) *\left\{\sum_{n=-\infty}^{\infty} g(n T) \delta(t-n T)\right\}
\end{aligned}
$$

Note that the term in the brackets on the right-hand side of the equation is the ideally sampled version as expressed in Eq. (3.1). Using the convolution property of the Fourier transform, the Fourier transform of flat-top sampled signal $s(t)$ will be the Fourier transform of the ideally sampled signal multipled by the Fourier transform of $p(t)$. This is shown as follows:

$$
\begin{aligned}
S(f)= & \text { FOURIER }\{p(t)\} \cdot \text { FOURIER }\left\{\sum_{n=-\infty}^{\infty} g(n T) \delta(t-n T)\right\} \\
& =P(f) \cdot\left\{f_{s} \sum_{n=-\infty}^{\infty} G\left(f-n f_{s}\right)\right\} \\
& =f_{s} \sum_{n=-\infty}^{\infty} P(f) G\left(f-n f_{s}\right)
\end{aligned}
$$


where $P(f)=\tau \frac{\sin (\pi f \tau)}{\pi f \tau} e^{-j \pi f \tau}$ is the Fourier transform of $p(\mathrm{t})$. It can be seen that the flat-top sampling introduces amplitude distortion and time delay, and the primary effect of $\mathrm{P}(f)$ is the attenuation of high-frequency components, known as the aperture effect. The aperture effect can be compensated for with an equalizing filter having a frequency response $1 / \mathrm{P}(f)$. If the pulse duration $(\tau)$ is much less than the sampling interval $(T)$, the $\mathrm{P}(f)$ is essentially constant over the band of interest and no equalization is needed.

\subsection{Bandpass Sampling}

A signal $\mathrm{s}(\mathrm{t})$ is called a bandpass signal if its Fourier transform $\mathrm{S}(f)=0$ except for $f_{1}<|f|<f_{2}$, as illustrated in Figure 3.4(a). An RF/IF radio signal is considered to be such a bandpass signal. Assuming the bandpass signal is ideally sampled at rate $f_{\mathrm{s}}$, the spectrum of the sampled signal becomes a periodic function like Eq. (3.4), and is shown in Figure 3.4(b).
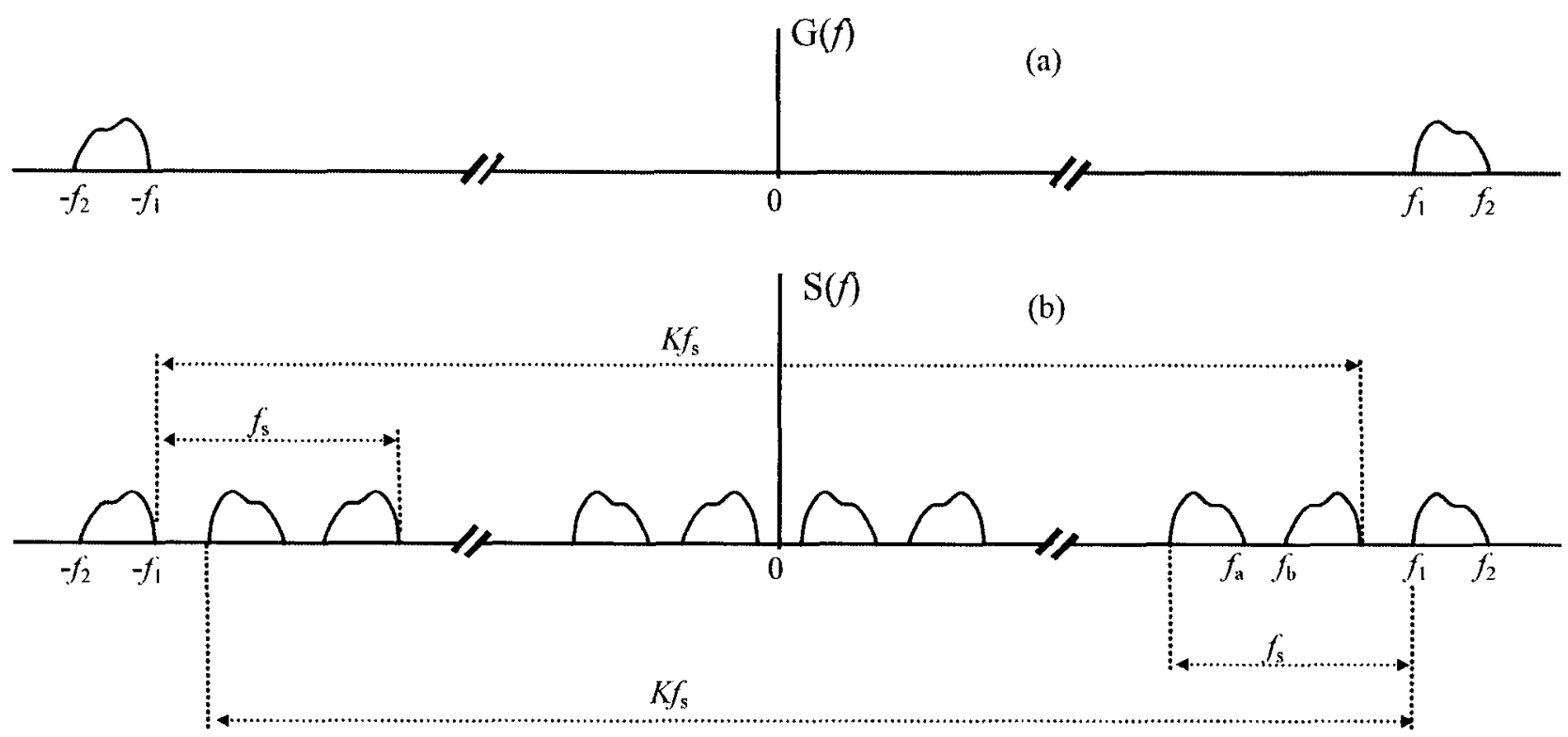

Fig. 3.4 Spectrum of (a) a Bandpass Signal and (b) its Sampled Signal 
Because of the periodicity, if any three adjacent images at any location do not overlap, there will be no overlap anywhere in the entire spectrum. Examining the three images on the right side of Figure $3.4(\mathrm{~b})$, the condition to avoid overlap is $f_{\mathrm{b}} \geq f_{\mathrm{a}}$, that is:

$$
K f_{s}-f_{2} \geq f_{2}-f_{s}, \quad K=\operatorname{Int}\left(2 f_{1} / f_{s}\right)
$$

where $\operatorname{Int}($.$) is the integer function. Note the use of K=\operatorname{Int}\left(2 f_{1} / f_{s}\right)$ already ensures that the image at $f_{\mathrm{b}}$ will not clash with the one in $\left(f_{1}, f_{2}\right)$. Equation (3.11) can be rewritten as:

$$
\left[\operatorname{Int}\left(2 f_{1} / f_{s}\right)+1\right] f_{s} \geq 2 f_{2}
$$

Note that when $f_{1}<f_{s} / 2$, the equation reduces to the lowpass sampling case. Hence, Eq. (3.12) is actually the aliase-free sampling condition for both lowpass and bandpass samplings. The above equation can be rewritten as follows:

$$
\left\{\operatorname{Int}\left[\frac{2 f_{2} /\left(f_{2}-f_{1}\right)-2}{f_{s} /\left(f_{2}-f_{1}\right)}\right]+1\right\} \frac{f_{s}}{f_{2}-f_{1}} \geq 2 \frac{f_{2}}{f_{2}-f_{1}}
$$

The graphical representation of above equation is illustrated in Figure 3.5, in which the lowpass sampling region is shown on the left of the Nyquist sampling rate line. It also shows that there are many aliase-free bandpass sampling zones though they get smaller as the sampling rate decreases. The theoretical minimum sampling rate $f_{\mathrm{s}}=2\left(f_{2}-f_{1}\right)$, called second-order sampling, occurs at the tips of the wedges, and corresponds to integer band positioning, i.e. $f_{2}=\mathrm{n}\left(f_{2}-f_{1}\right)$. The second-order sampling is risky, since any engineering imperfection will move the sampling into an aliasing area. 


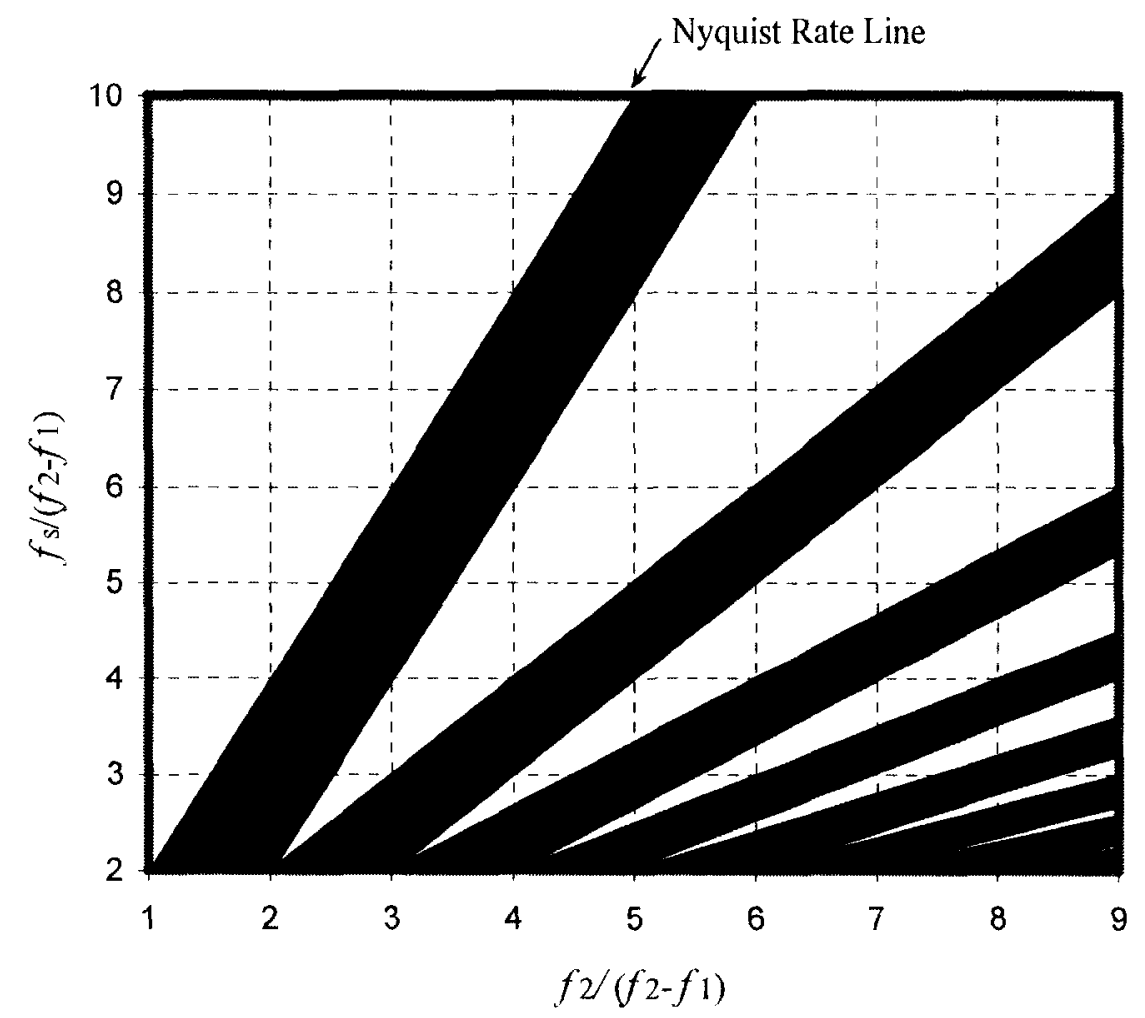

Fig. 3.5 Alias-free Sampling Zones (white) for Both Lowpass and Bandpass Sampling

\subsubsection{Quadrature Sampling for Direct-Conversion}

General reconstruction of a bandpass sampled signal is not trivial, and involves nonuniform sampling [8]. More importantly, the exact reconstruction of a radio signal waveform may not be in the best interest of radio technology. Rather, we just want to get the correct bits out, a process called demodulation. As will be reviewed in the next chapter, one important step in a receiver is to direct-convert the radio signal to baseband. As a special bandpass sampling scheme, quadrature sampling can serve this purpose. We will review this technique in this section with some new insight into the sampling rate and aliase-free condition. The quadrature sampling has a uniform sampling interval as follows: 


$$
T=\frac{k}{2 f_{c}}-\frac{1}{4 f_{c}}
$$

where $f_{\mathrm{c}}$ is the carrier frequency of the radio signal and $k$ is a positive integer. This gives the spectral image locations as shown in Figure 3.6. For clarity, only the lower images are shown. It can be seen that the quadrature sampling fixes the image's carrier frequency at $\left(n f_{\mathrm{s}} \pm f_{\mathrm{s}} / 4\right)$.

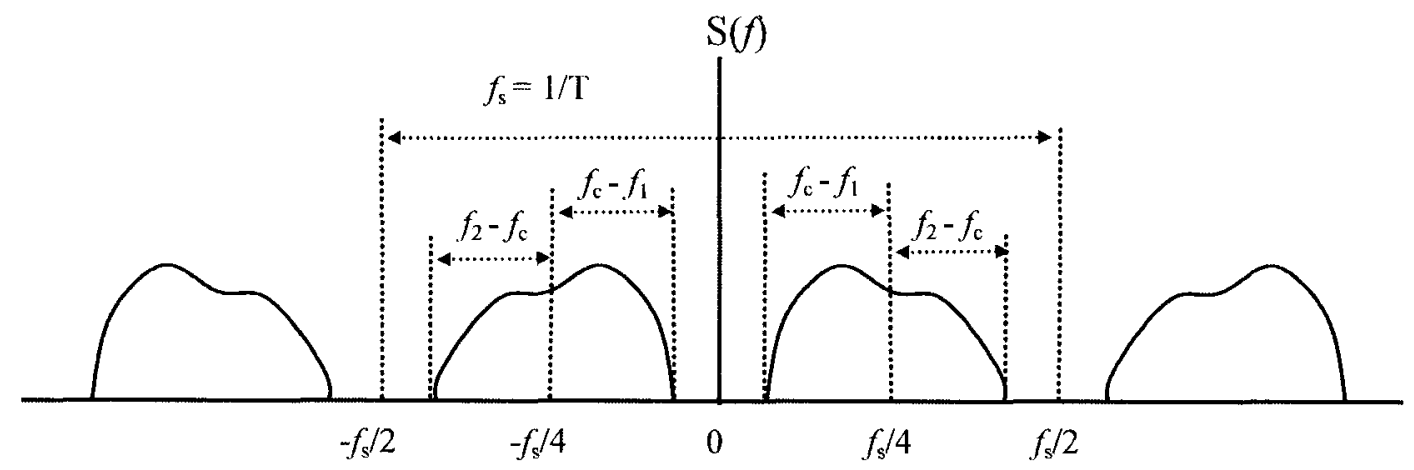

Fig. 3.6 Quadrature Sampling Image Location.

With the image's carrier frequency fixed, we have the following aliasing-free condition:

$$
\begin{aligned}
& 4\left(f_{\mathrm{c}}-f_{1}\right)<1 / T \\
& 4\left(f_{2}-f_{\mathrm{c}}\right)<1 / T
\end{aligned}
$$

It can be mathematically proven that (3.14) and (3.15a,b) ensure the general aliasing-free condition (3.12). This proof is developed in appendix A.

The quadrature sampling directly converts the signal to baseband and also allows easy reconstruction of original bandpass signal. A radio signal can be written as follows:

$$
g(t)=V_{l}(t) \cos \left(2 \pi f_{c} t\right)-V_{Q}(t) \sin \left(2 \pi f_{c} t\right)
$$


The sampled version will be:

$$
g(n T)=V_{l}(n T) \cos \left(2 \pi f_{c} n T\right)-V_{Q}(n T) \sin \left(2 \pi f_{c} n T\right)
$$

Combining (3.14) and (3.17) gives:

$$
g(n T)=V_{l}(n T) \cos \left[\pi n\left(k-\frac{1}{2}\right)\right]-V_{Q}(n T) \sin \left[\pi n\left(k-\frac{1}{2}\right)\right]
$$

Splitting the above sample sequence into odd-numbered and even-numbered terms produces:

$$
\begin{aligned}
& g(2 m T)=(-1)^{m} V_{I}(2 T m) \\
& g[(2 m-1) T]=(-1)^{m+k+1} V_{Q}\left[2 T\left(m-\frac{1}{2}\right)\right]
\end{aligned}
$$

Rearranging the two equations gives:

$$
\begin{aligned}
& V_{l}(2 T m)=(-1)^{m} g(2 m T) \\
& V_{Q}\left[2 T\left(m-\frac{1}{2}\right)\right]=(-1)^{m+k+1} g[(2 m-1) T]
\end{aligned}
$$

It is seen that lowpass I/Q signal samples are obtained from the sampling process. This is exactly the same as a direct quadrature down-conversion to baseband (or zero-IF), however with the data rate being decimated by a factor of 2 for each $\mathrm{I} / \mathrm{Q}$ component. Since the highest frequency component of $V_{l}(t)$ and $V_{Q}(t)$ will be the maximum one between $\left(f_{2}-f_{\mathrm{c}}\right)$ and $\left(f_{\mathrm{c}}-f_{1}\right)$, if conditions $(3.15 \mathrm{a}, \mathrm{b})$ hold, the lowpass sampling condition (Nyquist criterion) is met for these samples (whose sampling rate is now 1/2T). $V_{I}(t)$ and $V_{Q}(t)$ can therefore be reconstructed from their samples $V_{I}(2 T m)$ and $V_{Q}[2 T(m-$ 1/2)] according to the lowpass sampling reconstruction formula, Eq. (3.5): 


$$
\begin{aligned}
& V_{l}(t)=\sum_{m=-\infty}^{\infty} V_{l}(2 m T) \frac{\sin \left[\frac{\pi}{2 T}(t-2 m T)\right]}{\frac{\pi}{2 T}(t-2 m T)} \\
& V_{Q}(t)=\sum_{m=-\infty}^{\infty} V_{Q}(2 m T-T) \frac{\sin \left[\frac{\pi}{2 T}(t-2 m T+T)\right]}{\frac{\pi}{2 T}(t-2 m T+T)}
\end{aligned}
$$

From $(3.20),(3.21)$ and $(3.16)$, the bandpass signal $g(t)$ can be reconstructed as follows $[7]:$

$$
g(t)=\sum_{n=-\infty}^{\infty} g(n T) \frac{\sin \left[\frac{\pi}{2 T}(t-n T)\right]}{\frac{\pi}{2 T}(t-n T)} \cos \left[2 \pi f_{c}(t-n T)\right]
$$

The conditions for aliase-free sampling that are formulated by (3.14), (3.15a) and (3.15b) are more general than those in references [7][8]. As discussed in APPENDIX A, the conditions in references [7][8] are special cases of (3.14), (3.15a) and (3.15b). The quadrature sampling conditions in [7][8] are based on integer band second-order sampling. To use it safely, guard bands have to be added. In addition, the special integer band position and second-order sampling have to be arranged properly, whereas (3.14), (3.15a) and (3.15b) are not subject to these restrictions.

\subsubsection{Bandpass Sampling as Down-Conversion}

Also of interest in radio applications, is bandpass sampling as a general means of downconversion. Two possible down-conversions are reviewed in this section. Assume the sampling condition Eq. (3.12) is met, and that a radio signal is given by:

$$
g(t)=V(t) \cos \left[2 \pi f_{c} t+\theta(t)\right]
$$

Its spectrum can be expressed as: 


$$
G(f)=\frac{1}{2}\left[G_{B}\left(f-f_{c}\right)+G_{B}^{*}\left(-f-f_{c}\right)\right]
$$

where $G_{B}(f)$ is the spectrum of the complex envelope (equivalent lowpass signal) of the signal $\mathrm{s}(\mathrm{t})$, i.e. $V(t) \exp [\mathrm{j} \theta(\mathrm{t})]$, and ${ }^{*}$ is the conjugate operator. Assume $G(f)=0$ except for $f_{1}<|f|<f_{2}$

\section{Case (1) $\operatorname{Int}\left(2 f_{\mathrm{l}} / f_{\mathrm{s}}\right)=2 N$}

In this case, the spectrum $S(f)$ of the sampled version consists of spectral images of $\mathrm{G}(f)$, as illustrated in Figure 3.7. Note that $G_{B}(f)$ is on the right whereas $G_{B}{ }^{*}(-f)$ on the left in the frequency range from $-f_{\mathrm{s}} / 2$ to $f_{\mathrm{s}} / 2$. When filtered with an ideal lowpass filter with a cut-off frequency at $f_{s} / 2$, the spectrum becomes

$$
G_{L P}(f)=\frac{1}{2}\left[G_{B}\left(f-f_{c}+\frac{K}{2} f_{s}\right)+G_{B}^{*}\left(-f-f_{c}+\frac{K}{2} f_{s}\right)\right]
$$

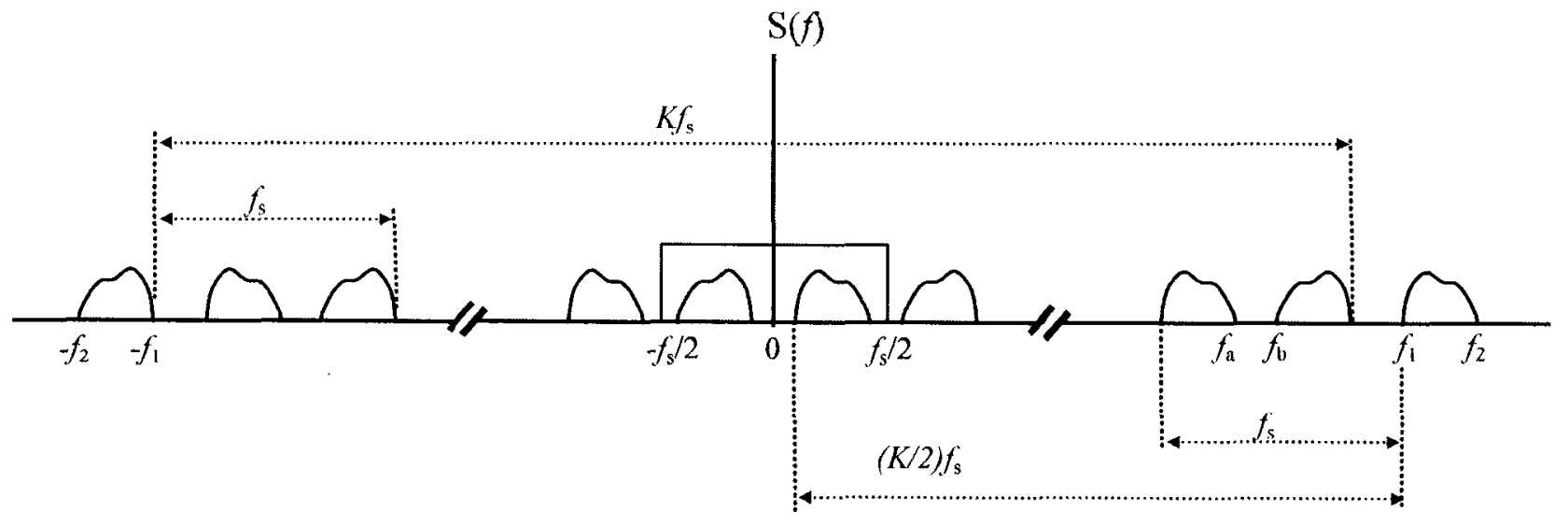

Fig. 3.7 Spectrum of Sampled Bandpass Signal For K=2N. 
The corresponding lowpass signal will be

$$
g_{L I}(t)=V(t) \cos \left[2 \pi\left(f_{c}-\frac{K}{2} f_{s}\right) t+\theta(t)\right]
$$

Comparing (3.26) with (3.23), we see that down-conversion is achieved without changing the spectral content of the signal.

Case (2) $\operatorname{Int}\left(2 f_{1} / f_{\mathrm{s}}\right)=2 N+1$

The spectral images are shown in Figure 3.8. Note that $G_{B}(f)$ is on the left whereas $G_{B}{ }^{*}(-$ $f$ ) is on the right in the frequency range from $-f_{\mathrm{s}} / 2$ to $f_{\mathrm{s}} / 2$. When filtered with an ideal lowpass filter with cut-off frequency at $f_{\mathrm{s}} / 2$, the spectrum becomes:

$$
G_{L I}(f)=\frac{1}{2}\left[G_{B}\left(f-f_{c}+\frac{K+1}{2} f_{s}\right)+G_{B} *\left(-f-f_{c}+\frac{K+1}{2} f_{s}\right)\right]
$$

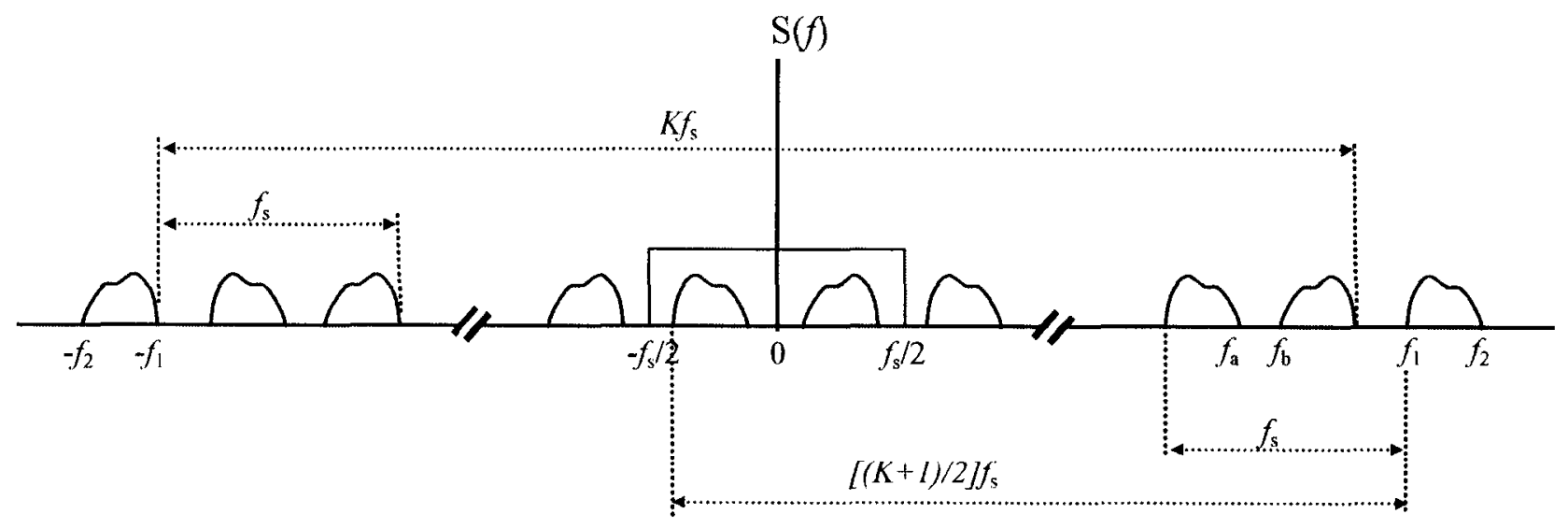

Fig. 3.8 Spectrum of Sampled Bandpass Signal for $\mathrm{K}=2 \mathrm{~N}+1$ 
The corresponding signal will be:

$$
\begin{aligned}
g_{L I}(t)= & V(t) \cos \left[2 \pi\left(f_{c}-\frac{K+1}{2} f_{s}\right) t+\theta(t)\right] \\
& =V(t) \cos \left[2 \pi\left(\frac{K+1}{2} f_{s}-f_{c}\right) t-\theta(t)\right]
\end{aligned}
$$

Down-conversion is therefore achieved, but with the phase reversed.

\subsection{Quantization}

A sampled signal is discrete in time, but not necessarily so in amplitude. In this case it should not yet be considered digital. Making the sampled signal discrete in amplitude, an important step in $\mathrm{A} / \mathrm{D}$ conversion, is accomplished by quantization, in which the amplitude is forcefully set to a number of prescribed levels. It is like a rounding operation and hence unavoidably introduces rounding errors, often called quantization errors, or quantization noise when it behaves like noise. The quantization happens not only in $\mathrm{A} / \mathrm{D}$ conversion, but also in purely digital computations where less significant bits are thrown away due to limited bit length.

Arithmetically, quantization is a rounding operation. There are two popular types of quantizer, mid-rise and mid-tread, as illustrated in Figure 3.9. They can be expressed as follows:

$$
\begin{array}{ll}
\text { Mid-rise: } & y=q[\text { floor }(x / q)+0.5] \\
\text { Mid-tread: } & y=q[\text { floor }(x / q+0.5)]
\end{array}
$$

where $q$ is the quantization step and floor(.) is a function that returns the greatest integer less than or equal to its argument. 

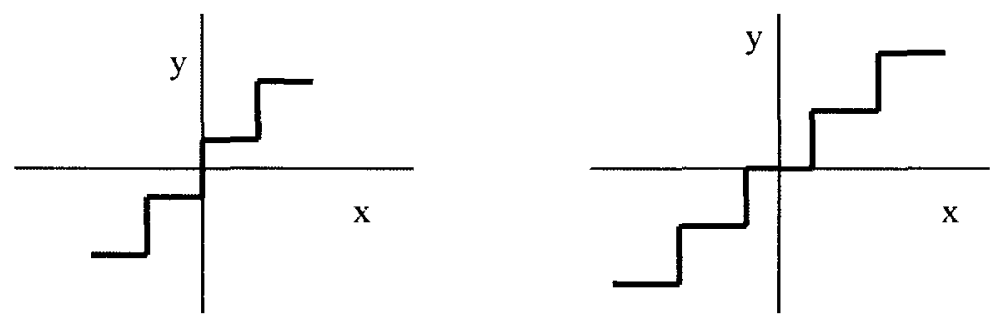

Fig. 3.9 Mid-Rise Quantizer (left) and Mid-Tread Quantizer (right)

The rounding operation is nonlinear by nature, and so is the quantization error. It should be noted that the quantization error is also strictly input-dependent. Generally, an exact analysis of the quantization error is extremely difficult. Efforts have been made to investigate the quantization error statistically [66][67][68]. It has been found that the conditions required to treat the quantization error as independent additive white noise are very strict, and cannot easily be satisfied in practical applications. At best, the quantization error can only be approximated as white noise. A useful observation is that as the number of quantization levels increases, the psd (power spectrum density) of the error signal resembles more and more the flat psd of an ideal white random signal [63]. Such a flat psd can be seen in commercial Nyquist-rate A/D converters. However, nonlinear products such as spurs exist nevertheless. One popular remedy to this problem is dithering, which randomizes the spurs and spreads them into noise.

Assuming an independent additive white noise with a uniform distribution with a step of $q$, the variance of the quantization noise can be obtained as follows:

$$
\sigma^{2}=\int_{-/ 2}^{y / 2} x^{2} \frac{1}{q} d x=\frac{q^{2}}{12}
$$

For a sinusoidal signal, the maximum signal swing will be $\left(q \cdot 2^{N}\right)$ where $N$ is the number of bits. The rms of the signal will be $\left(0.707 \cdot q \cdot 2^{N-1}\right)$. As a result, the maximum SNR or dynamic range for the quantizer will be: 


$$
S N R=20 \log \left[0.707 \cdot 2^{N-1} \cdot \sqrt{12}\right]=6 N+1.76 \quad(d B)
$$

It is worthwhile to note that the above formula is not valid for small $N$, say 1 or 2 , where the quantization is strongly nonlinear.

\subsection{Summary}

Basics of analog-to-digital conversion are reviewed and discussed in this chapter. A widely used sampling technique is the simple flat-top sampling, though it introduces an aperture effect. Bandpass sampling is of special interest in radio signal processing directly at IF \& RF; the challenge will be inevitably high-speed by nature, because a bandpass signal, although could be narrow in bandwidth, is a fast-changing signal. As the last stage of analog-to-digital conversion, quantization makes a sampled signal discrete in amplitude level. Quantization is a nonlinear operation. A weakly nonlinear quantization, where large number of quantization level is employed such as that in most Nyquist-rate A/D converters, can be characterized approximately in a linear manner; a strong nonlinear quantization however, where only small number of quantization level is employed such as that in sigma-delta modulation, cannot be analyzed linearly. In sigmadelta modulation, this kind of strong nonlinear quantization can be analyzed specifically, with a linear outcome, as will be discussed in chapter 5 . 


\section{CHAPTER 4 Digital Filtering and Sampling Rate Conversion}

In sigma-delta modulation, digital filtering and sampling rate conversion play important roles in converting fast over-sampled, short digital bits into Nyquist-rate, long ones. We will briefly review digital filtering techniques with emphasis on sampling rate conversion in this chapter.

\subsection{Digital Filter Basics}

A linear time-invariant (LTI) filter in a discrete-time system can be described by the following equation:

$$
y(n)=\sum_{k=-\infty}^{\infty} h_{k} x(n-k)
$$

where $x(n)$ is the input data stream, $y(n)$ is the output data stream, and $h_{k}$ is the impulse response of the filter. The discrete-time equation (4.1) is equivalent to its analog counterpart $y(t)=\int_{-\infty}^{\infty} h(\tau) x(t-\tau) d \tau$. More popular and practical is the following recursive equation that is equivalent to Eq. (4.1):

$$
y(n)=\sum_{k=0}^{N-1} a_{k} x(n-k)+\sum_{k=1}^{M} b_{k} y(n-k)
$$

A digital filter differs from an analog filter in that $b_{k}$ can be set to zero, resulting in a finite impulse response (FIR). This is impossible in the analog domain since, in practice, an analog filter's impulse response $h(t)$ is always infinite in duration. When $b_{k}=0$, we have: 


$$
y(n)=\sum_{k=0}^{N-1} a_{k} x(n-k)
$$

which is called a finite-impulse-response (FIR) filter, whereas the filter represented by (4.2) is called an infinite-impulse-response (IIR) filter.

The equivalent form of the Laplace transform $X(s)=\int_{-\infty}^{\infty} x(t) e^{-s t} d t$ in discretetime system is as follows:

$$
X(s)=\sum_{n=-\infty}^{\infty} x(n) e^{-s T n}
$$

where $T$ is the sampling interval. By making the following substitutions:

$$
z=e^{s T}=e^{\sigma+j \omega}=r e^{i 2 \pi f}
$$

where $f$ is the normalized frequency, the transform of (4.4) can be written as:

$$
X(z)=\sum_{n=-\infty}^{\infty} x(n) z^{-n}
$$

This is the standard form of the z-transform. Just as the Laplace or Fourier transform for an analog signal represents the signal and its impulse response in the $s$-domain or frequency-domain, the z-transform can be used to represent a digital signal and its impulse response in the z-domain. Performing the z-transform on Eq. (4.2), we have:

$$
H(z)=\frac{Y(z)}{X(z)}=\frac{a_{0}+a_{1} z^{-1}+a_{2} z^{-2}+\ldots+a_{N-1} z^{-(N-1)}}{1-b_{1} z^{-1}-b_{2} z^{-2}-\ldots-b_{M} z^{-M}}
$$


The frequency response can be obtained by replacing $z$ with $\exp (j \omega)$. The filter responses of Eq. (4.2) and (4.7) are often realized with the following structural model.

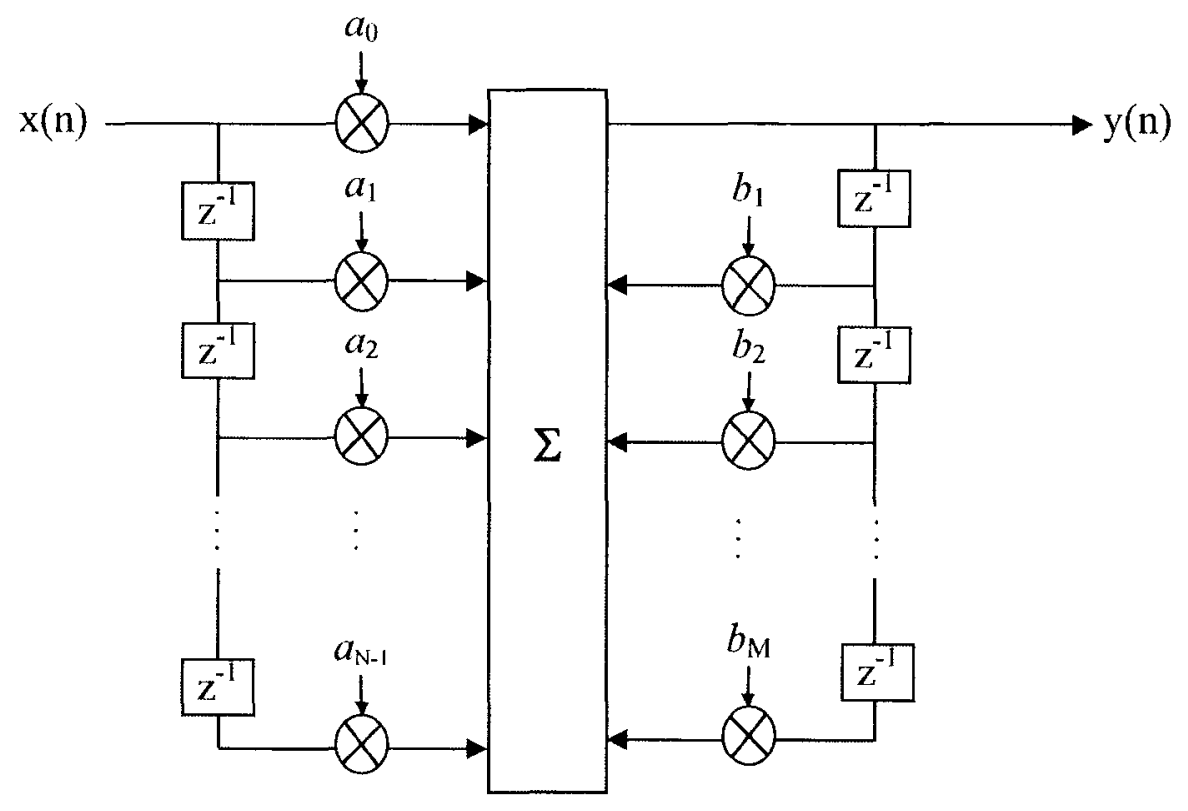

Fig. 4.1 Tapped-Delay-Line (Transversal) Model of a Digital Filter

Obviously, the FIR filter z-domain transfer function will be:

$$
H(z)=a_{0}+a_{1} z^{-1}+a_{2} z^{-2}+\ldots+a_{N-1} z^{-(N-1)}
$$

Similar to an analog filter transfer function that can be solely defined by its zcros and poles in the s-plane, a digital filter transfer function can be solely defined by its zeros and poles in the z-plane, as shown Figure 4.2. 
The z-plane and s-plane can be related to each other through the following bilinear transform, which is a conformal mapping of one complex plane into another complex plane.
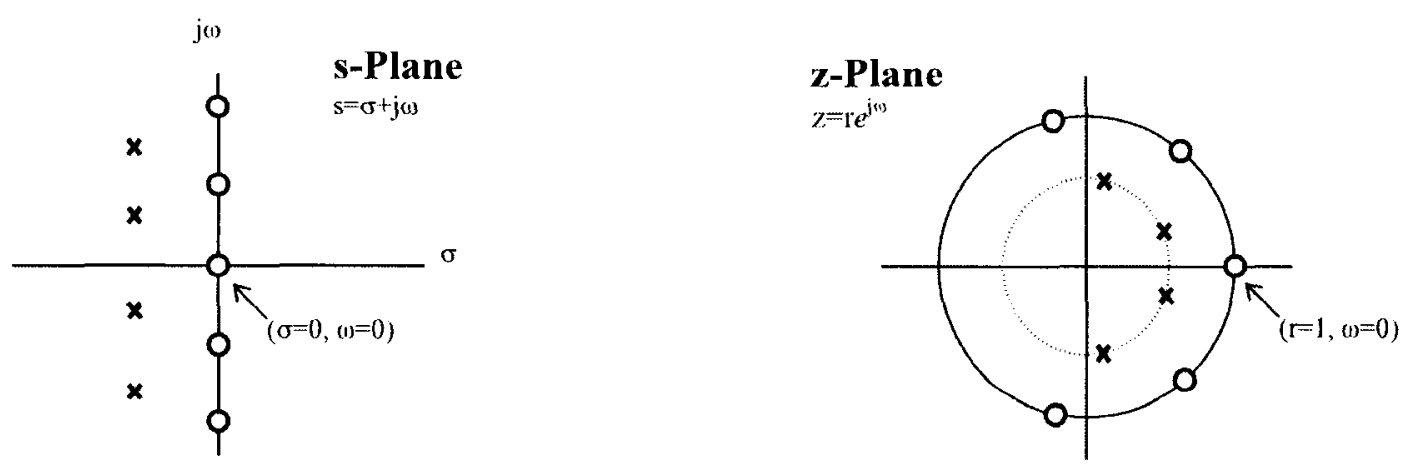

Fig. 4.2 S-domain and Z-domain

$$
s=\frac{2(z-1)}{K(z+1)}
$$

If $K=2 \tan (1 / 2)$, then the transform maps the s-domain's angular frequency range of 0 to 1 to the z-domain's angular frequency range of 0 to 1 . Thus an analog filter's transfer function in the s-domain can be transformed into digital filter's transfer function in the zdomain as follows:

$$
H_{\text {digital }}(z)=H_{\text {analog }}(s)=H_{\text {analog }}\left[\frac{2(z-1)}{K^{\prime}(z+1)}\right]
$$

Since the analog filter's response is always infinite, the corresponding digital filter will be of the IIR type. Therefore, an IIR digital filter design can start with an analog filter design and using (4.10).

An FIR filter, however, is purely digital without any analog equivalent. There are two types of FIR design methods. One is the windowing method. A window function is used to multiply a truncated version of an ideal infinite impulse response, which is obtained from a specified frequency response using an inverse Fourier Transform. The 
truncated version of the ideal infinite impulse response creates a ripple in the passband. The window function is used to smooth it. The windowing method is sub-optimal because it uses more coefficients than are required to reach a specified rejection in the stopband. A popular approach for FIR design is to try different coefficient values until an optimal set is obtained, as in the Remez searching algorithm.

The FIR filter is used more often than the IIR filter in communications because it is unconditionally stable and a linear phase response can be easily obtained. However, the FIR uses more coefficients than the IIR. Of critical concern in FIR design and implementation is the computational complexity in terms of the number of filter coefficients. Strategies include: (1) designing for as few coefficients as possible, (2) performing as few multiplications as possible, and (3) parallelism; doing as many multiplications simultaneously as possible. In the following discussion of a sampling rate conversion filter, we will see how the latter two strategies work.

\subsection{Sampling Rate Conversion Filtering}

Another major difference between digital and analog filters is that digital filtering can be done with sampling rate conversion. Digital filtering plus sampling rate conversion are equivalent to two separate functions in analog: filtering and frequency conversion. Sampling rate conversion can be down-sampling, often called decimation, or upsampling, often called interpolation. Thus, there are three types of conversion: (1) downsampling by an integer factor $\mathrm{D}$; (2) up-sampling by an integer factor $\mathrm{I}$; and (3) conversion by a rational factor I/D. In down-sampling by an integer factor, pre-filtering is needed to reject aliased frequencies. In up-sampling by an integer factor, post-filtering is needed to reject image frequencies. In conversion by a rational factor, both are needed. The conversion can be done by cascading the up- and down-sampling. However, better efficiency can be achieved by integrating them together. 


\subsubsection{Decimation Filter --- Down-Sampling by an Integer Factor}

Supposing the filter output is $y(\mathrm{i})$, the decimate-by-factor-D means that (D-1) samples in every $D$ samples will be thrown away, i.e. the actual output will be $y_{\mathrm{D}}(\mathrm{n})=y(D \mathrm{n})$. Note the sample that is kept among the D samples can be any one of them. For example, to decimate-by-3 on the data sequence $\{\ldots \mathrm{y} 1, \mathrm{y} 2, \mathrm{y} 3, \mathrm{y} 4, \mathrm{y} 5, \mathrm{y} 6, \mathrm{y} 7, \mathrm{y} 8, \mathrm{y} 9, \mathrm{y} 10, \ldots\}$, we can have $\{\ldots y 1, y 4, y 7, \ldots\}$, or $\{\ldots y 2, y 5, y 8, \ldots\}$, or $\{\ldots y 3, y 6, y 9, \ldots\}$. The three decimated results have the same information content once the filtering is properly performed, but they are different in the time delay. This actually means that the decimation filter can be used to adjust timing.

In reality, it is unnecessary to calculate those thrown-away (D-1) samples. Let us combine $y_{\mathrm{D}}(\mathrm{n})=y(D \mathrm{n})$ with Eq. (4.3):

$$
y_{D}(n)=\sum_{k=0}^{N-1} a_{k} x(D n-k)
$$

which is a skipped filtering. To save digital hardware, (4.11) can be re-organized as follows:

$$
\begin{aligned}
& y_{D}(n)=\sum_{k=0}^{N-1} a_{k} x(D n-k) \\
& =a_{0} x(D n)+a_{D} x[D(n-1)]+a_{2 D} x[D(n-2)]+\ldots \\
& +a_{1} x(D n-1)+a_{D+1} x[D(n-1)-1]+a_{2 D+1} x[D(n-2)-1]+\ldots \\
& +a_{2} x(D n-2)+a_{D+2} x[D(n-1)-2]+a_{2 D+2} x[D(n-2)-2]+\ldots \\
& +a_{b-1} x[D n-(D-1)]+a_{2 D-1} x[D(n-1)-(D-1)]+a_{3 b-1} x[D(n-2)-(D-1)]+\ldots
\end{aligned}
$$


This leads to a polyphase implementation. Each line in Eq. (4.12) is a sub-filter running at a rate down by a factor of D. An example of a decimate-by-three 7-tap FIR filter is shown in Figure 4.3. It is noted that the polyphase technique achieves efficiency by exploiting parallelism.

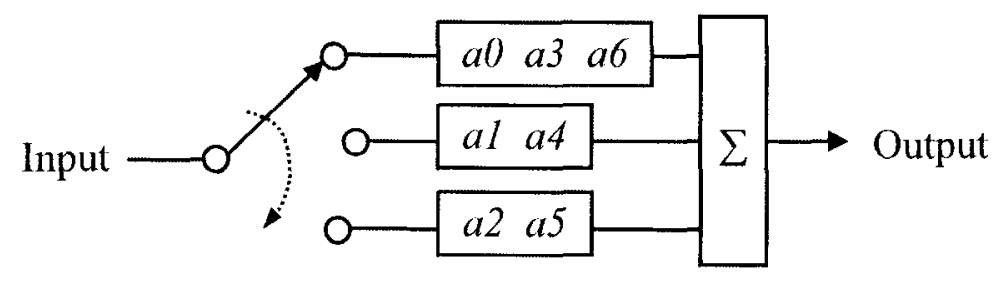

Fig. 4.3 A Decimate-by-Three Polyphase Filter Implementation

A widely used decimation filter in communications is the cascaded-integratorcomb (CIC) filter [39]. The principal building block of a CIC filter is the moving average filter, whose transfer function can be obtained from Eq. (4.8) by assuming all the coefficients to be unity:

$$
H(z)=1+z^{-1}+z^{-2}+\ldots+z^{-(N-1)}
$$

An important property of this filter is that it can be implemented as an IIR filter, since Eq. (4.13) can be rewritten as:

$$
H(z)=\frac{1-z^{-N}}{1-z^{-1}}
$$

The filter can be realized by the filter structure shown in Figure 4.1. This is shown in Figure 4.4. The left part of the filter structure is called the comb because it produces $\mathrm{N}$ zeros or notches; the right part is an integrator. The structure in Figure 4.4 can be rearranged as shown in Figure 4.5. 


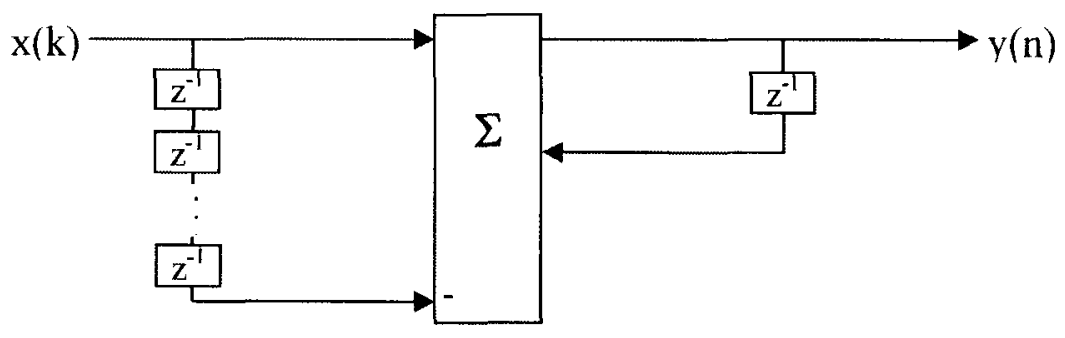

Fig. 4.4 A Single-Stage CIC Filter Structure

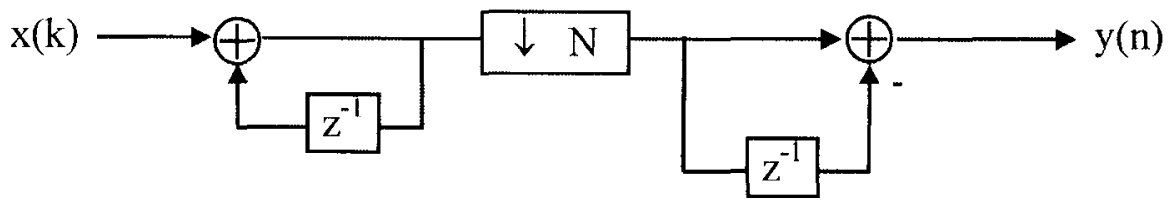

Fig. 4.5 A Single-Stage CIC Structure with a Down Sampler

Note that the comb and integrator are switched in position. This is like rearranging the order of the division and multiplication in Eq. (4.14). With $M$ such building blocks cascaded, the following transfer function can be realized:

$$
H(z)=\left(\frac{1-z^{-N}}{1-z^{-1}}\right)^{M}
$$

The filter structure will be as follows.

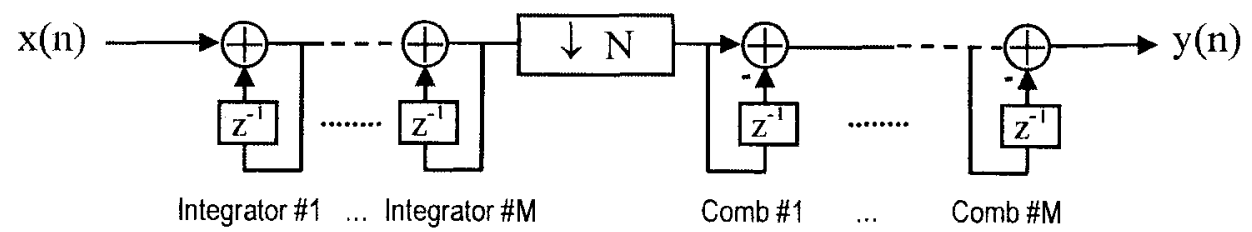

Fig. 4.6 Multi-Stage CIC Filter Structure 
The CIC filter is hardware efficient for a programmable decimation or interpolation filter, since it does not involve the storing of filter coefficients or the use of costly multipliers. Aliased or image bands can be placed to its stop band by correctly choosing the factor $\mathrm{N}$. The frequency response of an $M$-stage CIC filter can be obtained from Eq. (4.15) as follows:

$$
\left|H\left(e^{j 2 \pi f}\right)\right|=\left(\frac{\sin (\pi f N)}{\sin (\pi f)}\right)^{M}
$$

By simply choosing $N$ to be the decimation factor, the aliased bands will be located in the stopbands of the filter. One major drawback of the CIC filter is its droop in the passband. Compensation can be achieved, but at a cost of efficiency.

\subsubsection{Interpolation Filter --- Up-Sampling by an Integer Factor}

Decimation throws away some data samples. Interpolation, often called up-sampling, adds data samples. Interpolation by an integer factor I adds (I-1) samples between successive samples. The goal is to increase the sampling rate without affecting the spectrum of the signal. The interpolation filtering process can be done by adding (I-1) zeros between successive data samples, followed by image rejection filtering. The following figure shows an example of the spectral change when interpolation by a factor of three is performed. The spectrum is not changed by the zero padding, but four images appear in the new sampling window, as shown in Figure 4.7b. If the images are not filtered properly, distortion will occur in the time domain.

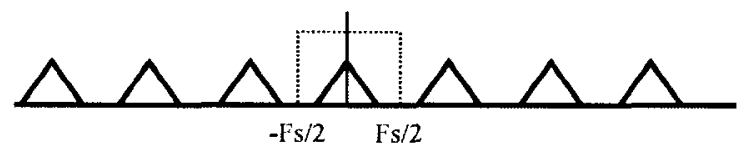

(a) Original signal

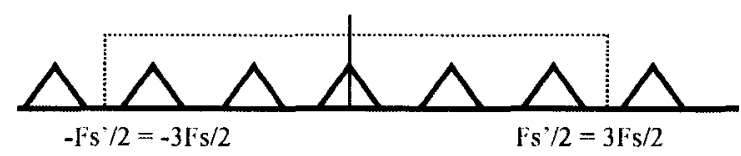

(b) Interpolate by three

Fig. 4.7 Spectral Impact of Interpolate-by-Three Using Zero Padding 
Mathematically, a data sequence $x(k)$ after interpolation by a factor I becomes:

$$
x_{I}(m)=\left\{\begin{array}{cl}
x(m / I), & m=0, I, 2 I, \ldots \\
0, & m=\text { others }
\end{array}\right.
$$

Assuming that the image-reject filter coefficients are $a(k)$, the interpolator output will be:

$$
y(n)=\sum_{k=0}^{N-1} a(k) x_{I}(n-k)
$$

The above two equations can be represented by following structure

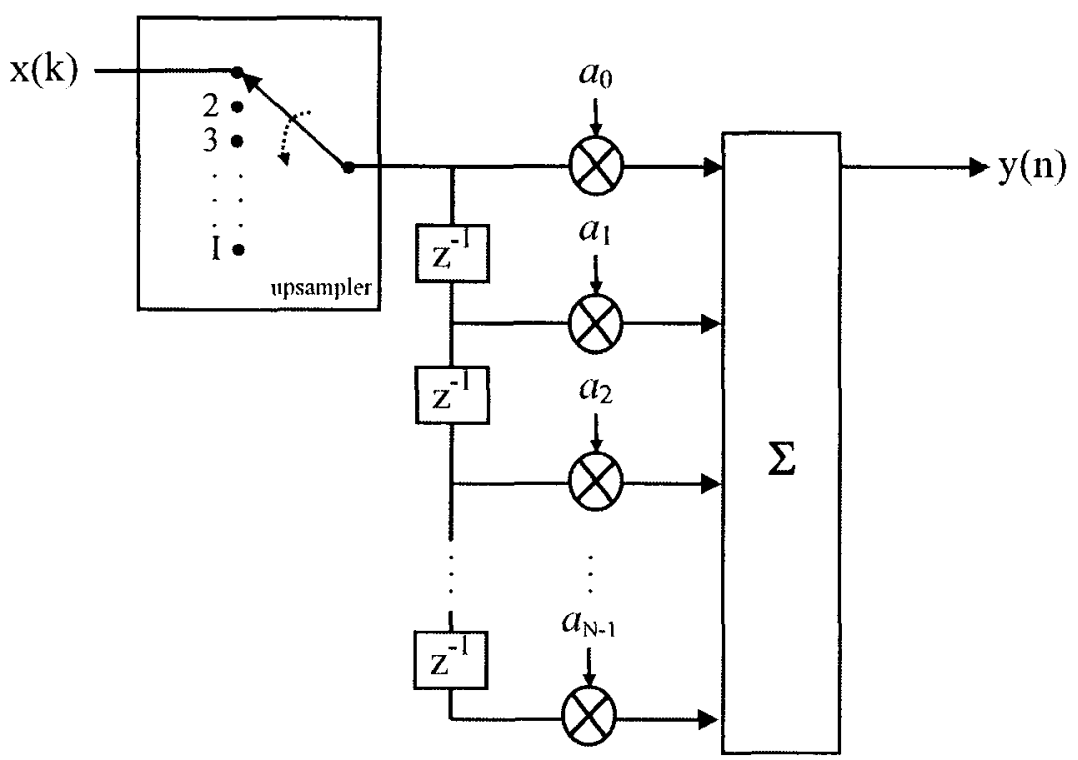

Fig. 4.8 Tapped-Delay-Line (Transversal Model) for Interpolation Digital Filter

Since $x_{I}(m)$ has (I-1) zeros in every I samples, by eliminating the zero terms, Eq. (4.18) can be broken down into following I-equations: 


$$
\begin{aligned}
& y(k I) \quad=a(0) x(k)+a(I) x(k-1)+a(2 I) x(k-2)+\ldots \\
& y(k I+1)=a(1) x(k)+a(I+1) x(k-1)+a(2 I+1) x(k-2)+\ldots \\
& y[k I+(I-1)]=a(I-1) x(k)+a(2 I-1) x(k-1)+a(3 I-1) x(k-2)+\ldots
\end{aligned}
$$

or equivalently it can be reduced to the following short but unfriendly form:

$$
y(n)=\sum_{j=0}^{M-1} a\left[n-\operatorname{Int}\left(\frac{n}{I}\right) I+j I\right] \cdot x\left[\operatorname{Int}\left(\frac{n}{I}\right)-j\right]
$$

where $M$ is the integer satisfying following inequality:

$$
\frac{N-1}{I}-\frac{n}{I}+\operatorname{Int}\left(\frac{n}{I}\right)<M \leq \frac{N-1}{I}-\frac{n}{I}+\operatorname{Int}\left(\frac{n}{I}\right)+1
$$

As a special case, when $N$ is an integer times $I, M$ will be equal to $N / I$. Whereas Eq. (4.20) may be efficient in programming, the equations in (4.19) are more human-friendly. The I equations mean that there are I samples out for each input sample. Eq. (4.19) and (4.20) can also be represented by a polyphase structure. The following figure shows an example of a 10-tap interpolate-by-three filter.

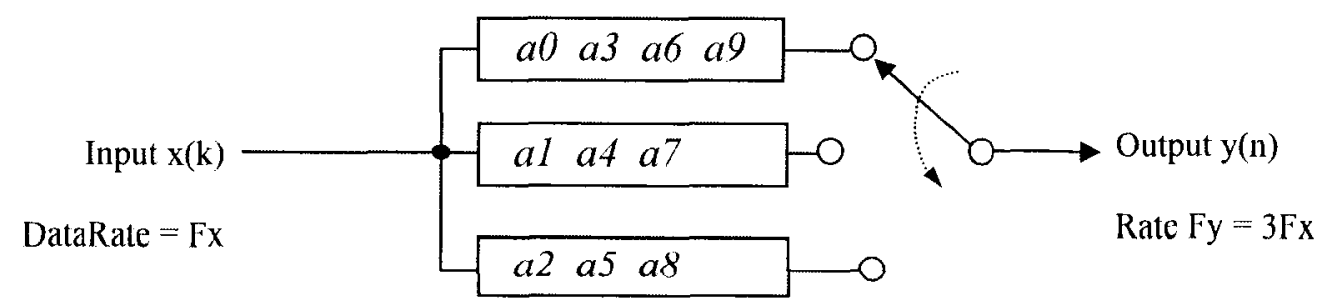

Fig. 4.9 A Interpolate-by-Three Polyphase Filtering Implementation 
Note that each sub-filter operates at an input data rate Fx, whereas a direct-form implementation of Figure (4.8) operates at the higher rate, since up-sampling is performed first. Also, the number of multiplications is reduced by a factor of $I$. Note that the polyphase implementation for the interpolator can be also viewed as a time-varying filter, in which the filter changes its coefficients at the output data rate.

\subsubsection{Sampling Rate Conversion by a Rational Factor}

A sampling rate conversion by a rational factor $I / D$ can be achieved by an interpolation of factor $I$ followed by a decimation of factor $D$. It is inefficient to do the interpolation and decimation separately, since both the image-reject and anti-aliasing filters between the interpolation and decimation would be performed at a rate $(I F x)$ where $F x$ is input data rate. The first thing to do is to combine the image-rejection filter $H_{1}(z)$ of the interpolator and the anti-aliasing filter $H_{D}(z)$ of the decimator as one $H(z)$. This is shown in the following figure. Assuming $H(z)$ has $N$ coefficients, the computational complexity in terms of multiplication per input sample for this implementation will be $N I$.

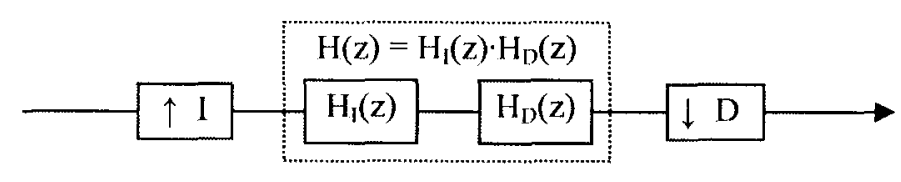

Fig. 4.10 Direct-Form by A Rational Factor $I / D$

One efficient approach is to perform the decimation with a polyphase scheme, as shown in the following figure. 


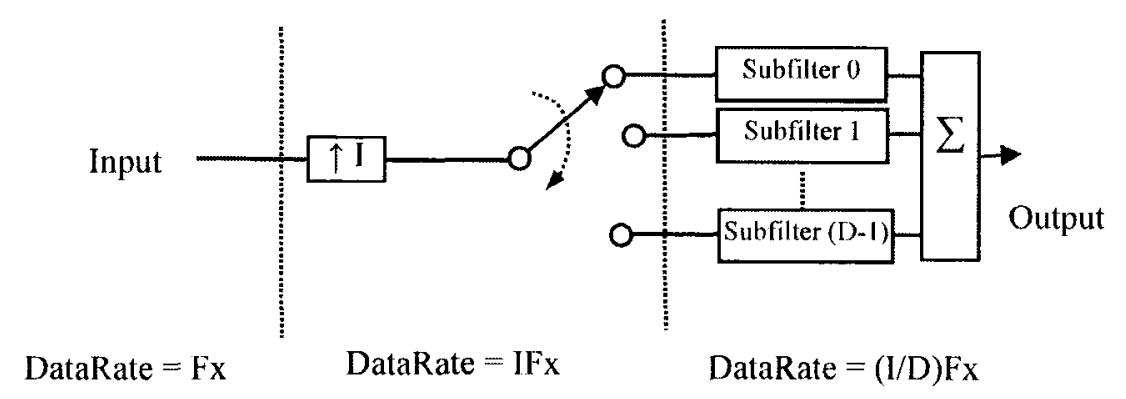

Fig. 4.11 An Efficient Way of Sampling Rate Conversion by A Rational Factor I/D

Here, $D$ sub-filters are obtained by breaking down $H(z)$. In this case, the number of multiplications performed per input sample will be $N L D$. Another approach is to perform the interpolation with a polyphase scheme, as shown in Figure 4.12.

Note the difference from Figure 4.11. Here $H(z)$ is broken into $I$ sub-filters, and the number of multiplications performed per input sample will be $N$. However, the most efficient structure can be obtained by moving the down-sampler forward, as is shown in Figure 4.13.

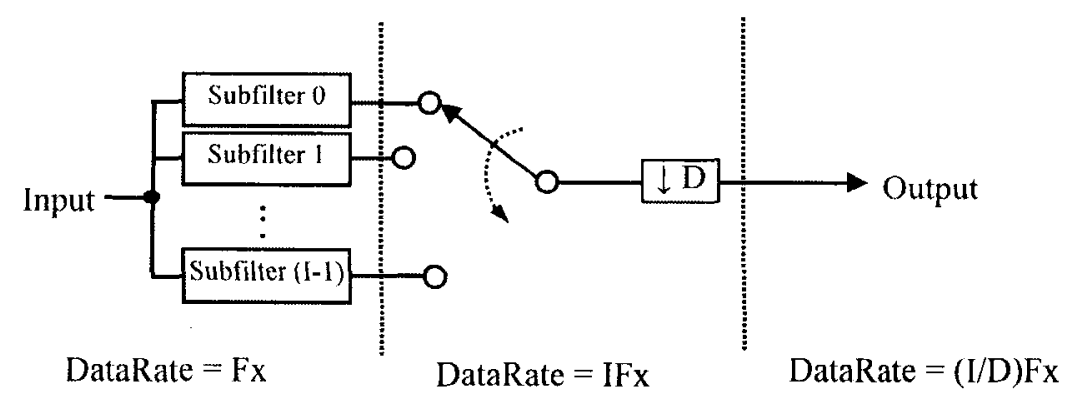

Fig. 4.12 Polyphase on Interpolator for Sampling Rate Conversion by A Rational Factor I/D 


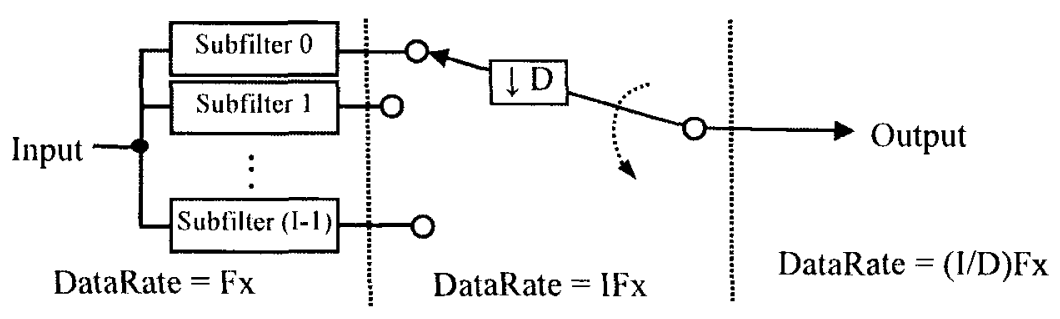

Fig. 4.13 The Most Efficient Way of Sampling Rate Conversion by A Rational Factor I/D

This is actually a skipped interpolation process. It can be represented more clearly with a clock structure model, as shown in Figure 4.14. The clock arm turns with a step of $D$. Since $I$ and $D$ are relatively prime, the turning period is $(I \cdot D)$ nodes or $D$ cycles, which means it takes $D$-cycles for the arm to come back to starting node. In other words, a node can only be picked once within $D$-cycles. Because the arm picks I nodes during the $D$ cycles, it is therefore proved that every node or every sub-filter must be picked once and only once in the $D$-cycle picking process.

Efficiency is achieved since only the picked sub-filters perform a filtering operation or computation. In the $D$-cycles there are $\mathrm{N}$ multiplications and there are $D$ data points in, so the average multiplication number for each input data is $N / D$.

This skipped interpolation is actually a re-ordering process. Assuming the original sub-filter order (without decimation) is $\mathrm{H}_{i}(\mathrm{z})$ where $i=0$ to (I-1) for each input sample, the new order will be $(i \mathrm{D})$ modulo $\mathrm{I}$, which is equal to $i \mathrm{D}-\operatorname{In} t(i \mathrm{D} / \mathrm{I}) \mathrm{I}$, where $\operatorname{Int}(x)$ denotes the integer part of $\mathrm{x}$. Take $I / D=5 / 3$ for example. The interpolate-by-tive is shown in Figure (4.15a), whereas for $I / D=5 / 3$, the arrangement will be like that shown in Figure (4.15b) 


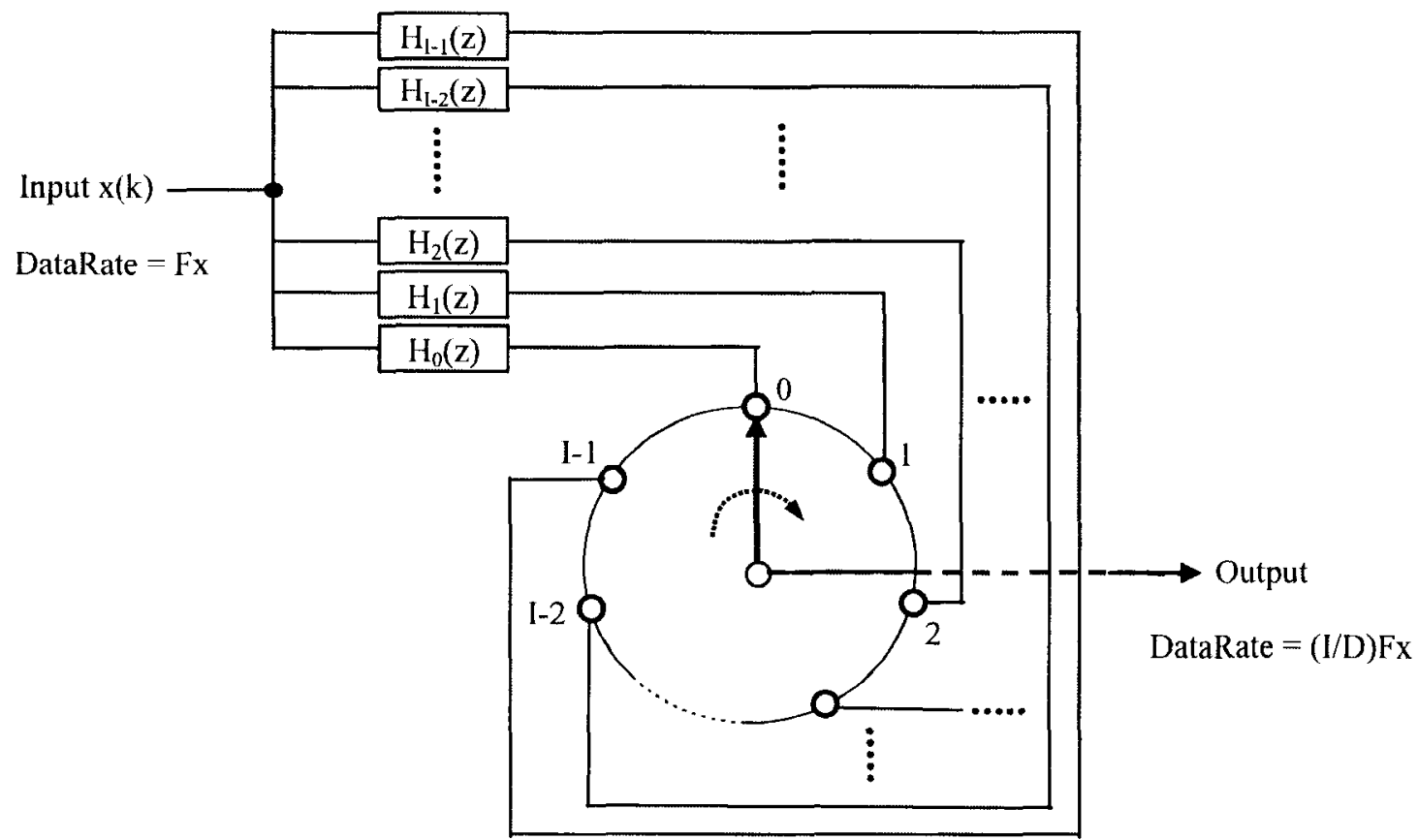

Fig. 4.14 Clock Structural Model for the Most Efficient Way of Sampling Rate Conversion by a Rational Factor I/D

A difference to note is that in Figure (4.15a) the picking arm picks all the five sub-filters for each input data, whereas in Figure (4.15b) the arm will pick two sub-filters for each of the first two input data, then only pick one sub-filter for the third input data.

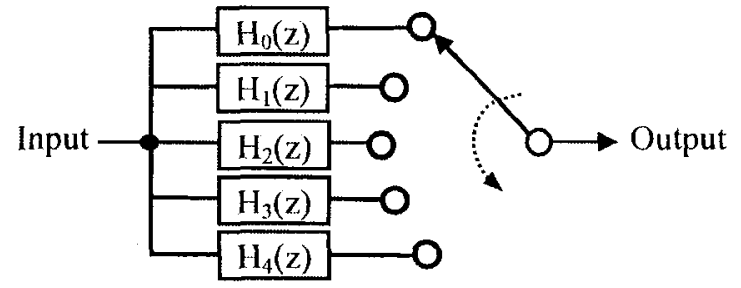

(a) Interpolate-by-5

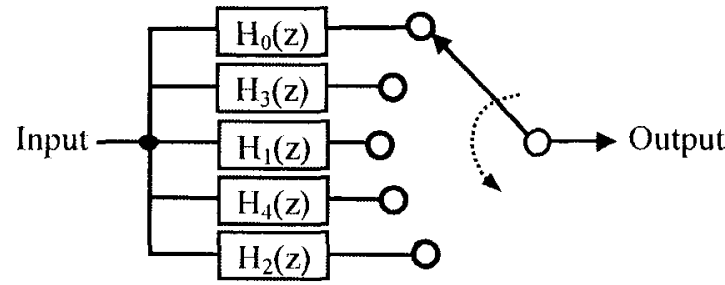

(b) $\mathrm{I} / \mathrm{D}=5 / 3$

Fig. 4.15 An Example of Sampling Rate Conversion by $5 / 3$ in Comparison with That by $5 / 1$ 
The following table summarizes the efficiency/complexity for the above approaches.

Table 4.1 Efficiency of the Skipped Polyphase Interpolator

\begin{tabular}{|c|c|c|}
\hline Implementation & Complexity & $\begin{array}{c}\text { Example } \\
\mathbf{I = 3 5}, \mathbf{D}=\mathbf{2 9}, \mathbf{N}=\mathbf{1 8 9}\end{array}$ \\
\hline Direct-form & NI & 6615 \\
\hline Polyphase Interpolator & N & 189 \\
\hline Polyphase Decimator & NI/D & 228 \\
\hline $\begin{array}{c}\text { Skipped Polyphase } \\
\text { Interpolator }\end{array}$ & N/D & 7 \\
\hline
\end{tabular}

\subsection{Summary}

A digital filter can be finite-impulse-response (FIR), a unique feature compared to its analog counterpart. A digital filter can be precisely designed and implemented because each coefficient in its impulse response can be changed independently, impossible in analog filter design. That is why the performance of the digital filter can be exceptionally good. However, it usually comes with large number of computations and high-power consumption. Sampling rate conversion in digital is equivalent to frequency conversion in analog. A new clock structural model for a rational factor, sampling-rateconversion digital filter is provided. The new model can be helpful in designing efficient sampling-rate-conversion digital filters. 


\section{CHAPTER 5 Noise Shaped Oversampling - Sigma-Delta Modulator}

The advantage of conventional Nyquist-rate $\mathrm{A} / \mathrm{D}$ conversion is a low sampling frequency that only needs to be twice the signal bandwidth. However, a large number of bits (many quantization steps) is needed in order to have a reasonable dynamic range. Recall from Section 3.4 that for a uniformly distributed quantization noise, the dynamic range is given by $(6 N+1.76) \mathrm{dB}$, where $N$ denotes the number of bits. $\mathrm{A} / \mathrm{D}$ conversion at the circuit level is essentially threshold detection performed with comparators. In practice, the quantization scale is bounded by the power supply level; therefore a large number of bits (many quantization steps) requires each step to be small. This in turn, requires high accuracy for the threshold level detection, requiring costly, precise analog components. For example, circuit components in a 16-bit (or higher) A/D converter usually need to be laser-trimmed.

$\mathrm{A} / \mathrm{D}$ conversion is defined by the sampling rate and the sampling bits (quantization steps). The Nyquist-rate $\mathrm{A} / \mathrm{D}$ conversion is just one of the choices, and is featured with a low sampling rate and a large number of bits (many quantization steps). The two variables (sampling rate and bits), ultimately require two different sets of hardware resources, that can be traded off. In terms of dynamic range, one sampling bit can be traded with four times the sampling rate, because both contribute to the same amount of dynamic range $(6 \mathrm{~dB})$. The extra sampling bits provide more dynamic range on the upper side while the oversampling rate does so on lower side (by reducing the quantization noise level). Therefore, straightforward oversampling can be employed to reduce the number of sampling bits and ease the accuracy requirement for the threshold level detection. The expense, however, will be timing jitter and clock feed-through 
arising from the higher clock rate. Consequently, there is no net gain in component performance for straightforward oversampling.

Noise-shaped oversampling, or sigma-delta modulation, by contrast offers a net gain in this trade-off because of its noise redistribution. In sigma-delta modulation, inband quantization noise is suppressed at the expense of increased out-of-band noise. Therefore, low in-band quantization noise can be achieved at a much lower clock rate than for a plain oversampling scheme, and at the same time with far fewer sampling bits (quantization steps) than for a Nyquist-rate sampling. This is accomplished through a feedback loop with a proper loop filter as illustrated in Figure 5.1. The encoder and decimator following the feedback loop transfer the data to Nyquist-rate without loss of dynamic range.

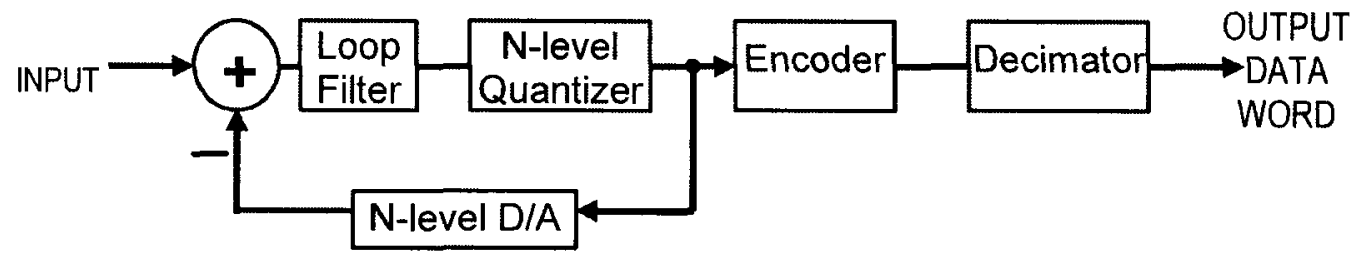

Fig. 5.1 A Sigma-Delta Modulator

The sigma-delta modulator (SDM) is a nonlinear feedback loop because of the nonlinear quantizer. In contrast to the weakly nonlinear quantization in the Nyquist-rate $A / D$ converter, the quantization in the SDM is strongly nonlinear due to the far fewer quantization steps employed. It is this strong nonlinearity that makes the SDM design a challenging task. 


\subsection{Stability of the Nonlinear Feedback Loop}

The concern for a nonlinear system is possible in stability. Stability seems intuitively trivial, yet the definition of stability is non-trivial. There are many definitions for stability, each with its own subtle nuances. A popular definition of stability is bounded input, bounded output (BIBO), which states that a system is stable if every bounded input produces a bounded output [73]. However, this definition is not applicable to the SDM loop, whose output is hard-limited by lower and upper bounds in the quantizer's scale. More appropriate in practice is the working definition, which states that a SDM is stable if its output is free of limiting cycles and/or strong repetitive patterns. In the stable state, the noise-shaping loop works and the output spectrum appears as a signal surrounded by large amounts of noise.

Exact analysis on the stability of a $1^{\text {st }}$-order loop can be performed as follows. The $1^{\text {st }}$-order loop is illustrated in Figure 5.2. Assuming a mid-rise quantizer with a unit quantization step for simplicity, we have:

$$
y_{n}=\operatorname{Floor}\left(w_{n}\right)+\frac{1}{2}
$$

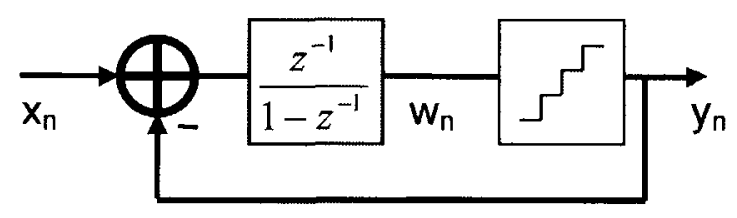

Fig. 5.2 A $1^{\text {st }}$-order SDM loop

where Floor(.) is the maximum integer that is less than or equal to the argument. The loop filter is an integrator that gives the following time-domain equation:

$w_{n}=w_{n-1}+x_{n-1}-y_{n-1}=w_{n-1}+x_{n-1}-\operatorname{Floor}\left(w_{n-1}\right)-\frac{1}{2}=w_{n-1}-\operatorname{Floor}\left(w_{n-1}\right)+x_{n-1}-\frac{1}{2}$ 
Since $0 \leq[\mathrm{x}-\mathrm{Floor}(\mathrm{x})]<1$, we have:

$$
x_{n-1}-\frac{1}{2} \leq w_{n}<x_{n-1}+\frac{1}{2}
$$

It follows that $\operatorname{Floor}\left(x_{n-1}-\frac{1}{2}\right) \leq \operatorname{Floor}\left(w_{n}\right) \leq \operatorname{Floor}\left(x_{n-1}+\frac{1}{2}\right)$. Therefore, from (5.1):

$$
\operatorname{Floor}\left(x_{n-1}-\frac{1}{2}\right)+\frac{1}{2} \leq y_{n} \leq \operatorname{Floor}\left(x_{n-1}+\frac{1}{2}\right)+\frac{1}{2}
$$

Eq. (5.4) shows a bounded output as long as the input is bounded. In practice, this holds true within the range of the quantizer's full scale. Beyond that, the Floor(.) function is no longer valid and hard-limiting occurs. Therefore, within the quantizer's full scale range, the loop can be designed successfully in terms of noise-shaping.

Exact analysis of higher-order loops is difficult. Yet, by simulation, it is not difficult to find a stable range for a SDM. For a 1-bit SDM, a rule of thumb is that the noise-shaping gain should be less than 2 [13].

\subsection{Linearity of the Nonlinear Feedback Loop}

A properly designed SDM performs noise-shaping, as predicted by its linear model. However, the SDM is inherently nonlinear, since the quantizer in the SDM is a nonlinear device whose output steps up or down when the input crosses any of its preset threshold levels. Quantization is equivalent to the rounding operation in arithmetic. The quantization error is strictly input-dependent and therefore deterministic. Though deterministic, it is difficult to handle in a deterministic way. It would make modelling and analysis much easier if it could be treated statistically as additive random noise. Efforts have been made to investigate the statistical properties of the quantization error. It has been found that the conditions required to treat the quantization error as independent additive white noise are very strict, and cannot easily be satisfied in practical applications. The quantization error of uniform distribution can at best, be approximated as white noise [66][67][68]. Nevertheless, the quantization error is often treated as 
additive white noise for practical reasons; the quantizer can then be modeled as a linear device, making linear analysis possible. Such an approximation for the Nyquist-rate A/D conversion works fine because the large number of sampling bits (many quantization steps) makes linear behaviour dominant, and non-linear behaviour a minor issue.

For noise-shaped oversampling or SDM, where only one or a few bits (a few quantization steps) are employed, the quantizer operates in a strongly non-linear manner. A linear model cannot be simply taken for granted. Only by examining the nature of the noise-shaped output and the loop feedback mechanism, can the validity of the linear model be explained. Stability is the starting point. By definition, specifically the working definition of stability, the output of the stable SDM loop is free of limiting cycles and/or strong repetitive patterns. Its power spectrum is noise-shaped and/or dominated by noise-like signals. In the time domain it corresponds to a highly randomized data sequence. The randomness is even further enhanced in practice by system noise and information-rich input signals (in place of simulation signals such as sinusoidal tones). The key is the feedback of such a randomized signal to the input of the quantizer. Recall that quantization error from the quantizer is strictly input-dependent. It follows that the randomness of the feedback signal makes the quantization error resemble random noise and hence makes the linear model valid. In other words, the randomized feedback signal acts as a dithering signal to the quantizer. On the other hand, linear behaviour of the nonlinear feedback loop, when properly designed, enforces the noiseshaping performance at the output. Therefore, the noise-shaping and the linear behaviour reinforce each other. In this way, linearity is accomplished for the strongly nonlinear feedback loop --- a beauty with the noise-shaped oversampling! Such a mechanism works fine and is sustainable as long as the loop is in a stable state. When the input to the loop increases, the output signal will gradually become deterministic and less random. And, above a certain level the loop can go unstable.

Figure 5.3 illustrates a linear model for the SDM, where the quantizer is modeled as an adder with quantization noise $(Q)$. The mismatch errors from the feedback DAC are also treated as additive white noise in the linear model. The transfer function for the linear model can be obtained as: 


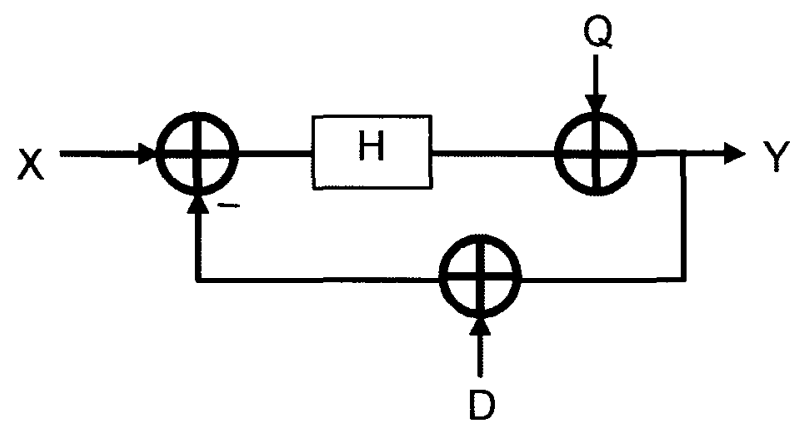

Fig. 5.3 Linear Model for a Sigma-delta Modulator (SDM)

$$
Y=\frac{H}{1+H}(X-D)+\frac{1}{1+H} Q
$$

where $H$ is the loop filter transfer function. The above equation shows that the signal and the quantization noise are treated differently. The noise-shaping comes from carefully choosing the loop filter so that the signal transfer function $H /(1+H)$ will allow the signal to pass, whereas the noise transfer function $1 /(1+H)$ will suppress the in-band quantization noise. The above equation also shows that the noise term $(D)$ from the feedback path is processed in exactly the same way as the signal, and is not suppressed. Therefore it is critical in SDM design to reduce the noise and mismatch errors on the feedback path.

\subsection{1-Bit Sigma-Delta Modulator}

A 1-bit SDM employs a single element for both the quantizer and the feedback DAC and is the simplest SDM that can be implemented. The quantizer is basically a comparator. The simplest example is a first-order lowpass SDM, whose loop filter is an integrator with transfer function $H=1 /(z-1)$. An all pass signal transfer function $\operatorname{STF}(\mathrm{z})=H /(1+H)$ $=1 / z$, and a high-pass noise transfer function $\operatorname{NTF}(z)=1 /(1+H)=1-z^{-1}$ is obtained. Therefore, noise at low frequencies is suppressed, and a lowpass signal will not be 
affected by otherwise high quantization noise. Computer simulation for this example is shown in Figure 5.4. A highpass noise-shaped spectrum is observed for a low frequency tone input signal.

A significant advantage of the 1-bit SDM is that the single element DAC is absolutely linear and therefore contributes no element mismatch error on the feedback path. However, the 1-bit SDM produces the most nonlinear operation in the quantizer. As a result, the loop can easily go unstable. This is especially true with high order loops, where high loop gain acting on the large quantization error forces the loop to enter limit cycles well before the input signal reaches its otherwise maximum full scale level. This severely reduces the dynamic range of the modulator. Again, the rule of thumb is that the NTF gain should be less than two in order to ensure stability [13].

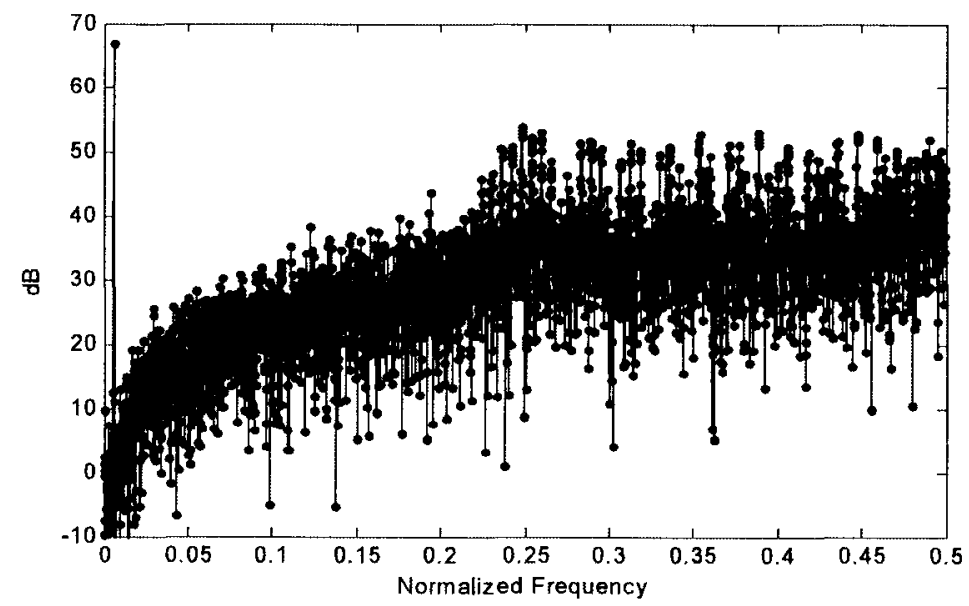

Fig. 5.4 Noise-Shaping of a 1-bit First-order Lowpass SDM

Increasing the clock rate (oversampling ratio) improves stability at the expense of increased power consumption, time jitter and clock feedthrough, and therefore should be used with caution. Other two widely used approaches are multi-stage SDM (MASH) and reduced loop gain, as will be discussed in the following. 


\subsubsection{Multi-Stage SDM (MASH)}

Multi-stage SDM (MASH) [1][3][41] employs multiple cascaded low-order loops that are unconditionally stable. Each stage takes the previous stage's quantization error as its input. A digital cancellation stage is placed at the output to cancel the lower order noise, and as a result the MASH structure achieves high-order noise shaping. An example of a second-order MASH is shown in Figure 5.5. The first order noise is cancelled at the output and the SDM achieves second-order quantization noise shaping. In principle, this can be extended to $\mathrm{m}^{\text {th }}$ order noise shaping while preserving unconditional stability. In practice, performance is limited due to component mismatches among stages resulting in imperferct noise cancellation [19].

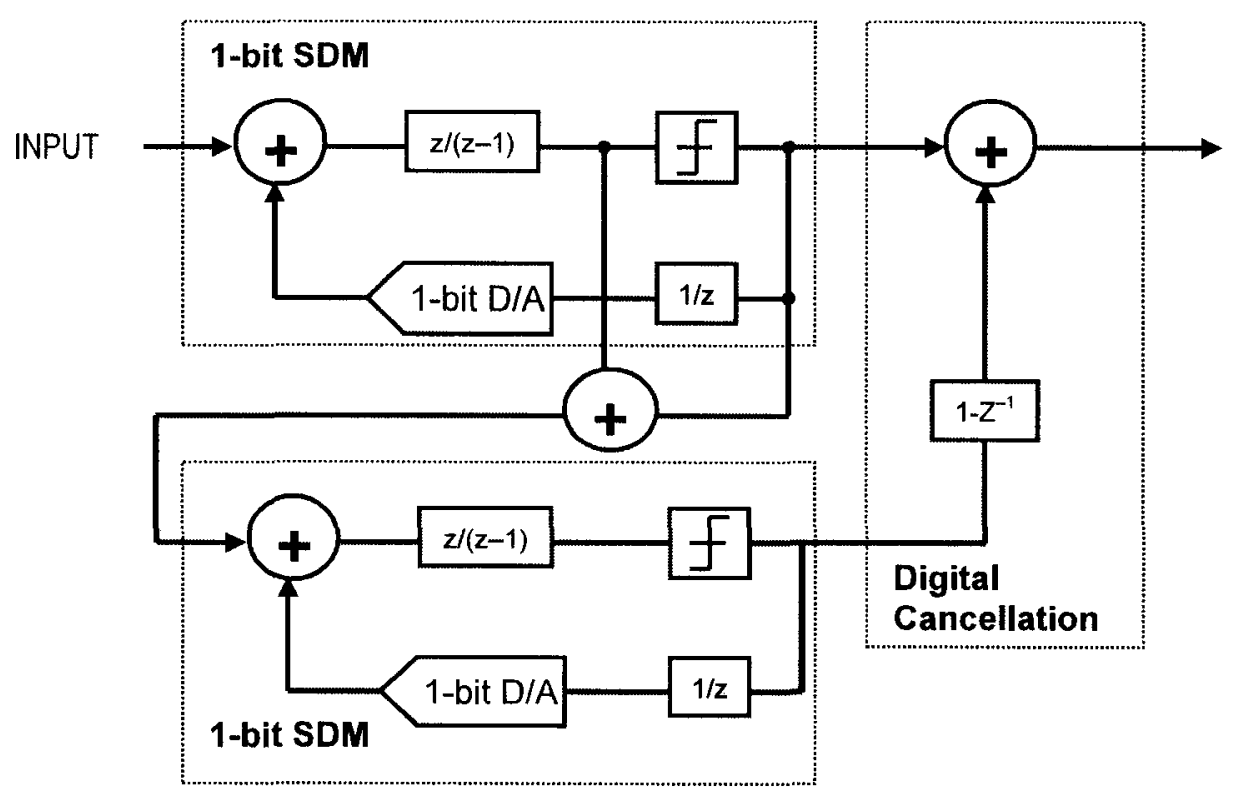

Fig. 5.5 A Multi-Stage (MASH) Lowpass Sigma-delta Modulator

Since the signal at the output of the digital cancellation stage becomes multi-bit, the MASH is, in fact, another way of doing multi-bit noise-shaping. However, it should be noted that the MASH does not solve the mismatch problem normally seen in multi-bit 
SDM; instead it transfers the problem from single-stage matching to multiple stages. Another limitation of the MASH structure is the increased settling time being the sum of each stage's settling time. This can limit the operating frequency significantly. Moreover, low-order 1-bit loops suffer from idle patterns and dead zones, causing repeating patterns at low signal levels, a phenomenon that rarely occurs for higher order loops [63].

\subsubsection{Reduced Gain for Stability}

Another common method for improving high-order 1-bit SDM stability is to reduce the noise-shaping gain intentionally. For example, for a standard full-gain sixth-order bandpass NTF $=\left(1+z^{-2}\right)^{3}$ to work in a 1-bit SDM, the noise-shaping gain needs to be reduced by $9.3 \mathrm{~dB}$ [64]. Multi-loop structures are often employed for the reduced gain loop. An example is shown in Figure 5.6 [1][3][54]. The coefficients $B_{1} B_{2} \ldots B_{m}$ and $K_{1} K_{2} \ldots$ $K_{m}$ are used to control the NTF gain.

However, what is often not noticed is a reduced bandwidth associated with the reduced-gain NTF. This is illustrated in Figure 5.7 where a narrow pass-band can be seen for the reduced-gain NTF. A quantitative analysis and simulation, presented in Section 5.5, shows a substantial loss of bandwidth arising from the reduced gain. This may not be too serious for lowpass or baseband SDMs, since their pass-band can be expanded by simply increasing the oversampling ratio (clock rate). However, design challenges such as power consumption, jitter and clock feedthrough come with increased clock rate. Moreover, for band-pass SDM, increasing the sampling clock shifts the passband higher. 


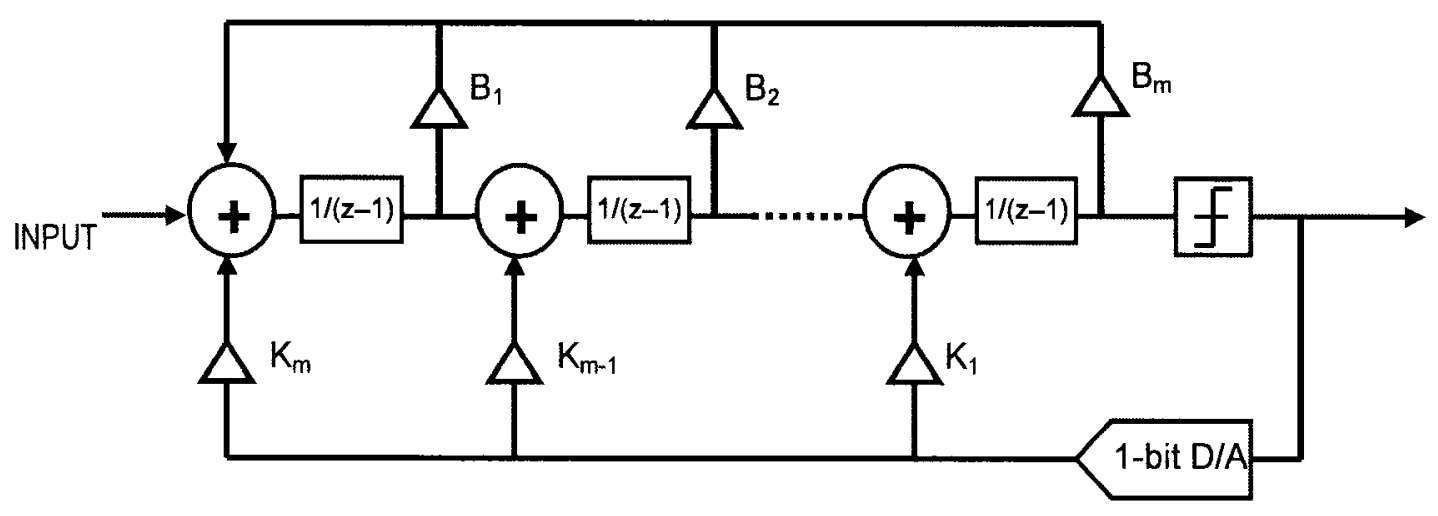

Fig. 5.6 An $m$ th-order Lowpass Sigma-delta Modulator

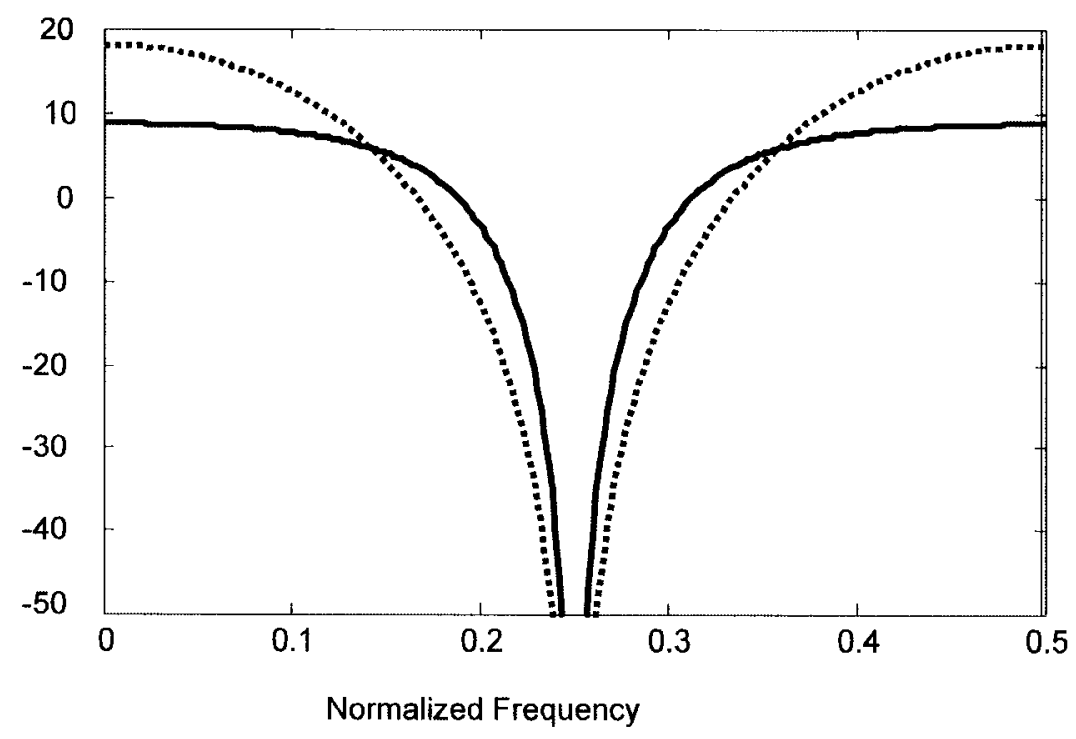

Fig. 5.7 Sixth-order Bandpass SDM NTF Full-Gain $\left(1+z^{-2}\right)^{3}$ (dotted line) and ReducedGain $\left(1+z^{-2}\right)^{3} /\left(1+0.43 z^{-2}\right)^{3}$ (solid line) 


\subsection{Multibit Sigma-Delta Modulator}

Multibit sigma-delta modulators have received growing interests recently [74]. A multibit SDM employs more than one element in both the quantizer and the feedback DAC. The improved linearity in the quantizer makes a much more stable loop and offers larger dynamic range at lower oversampling ratio. In addition, the design of the multi-bit SDM can be much simpler. Since a single loop and a single stage structure can be realized, a full-gain NTF can be implemented. Further, the adjustment of the loop time delay is a much easier task.

The multi-bit quantizer is a flash-type $\mathrm{A} / \mathrm{D}$ converter, which converts the analog signal into digital thermometer code. A thermometer code is a code where all of the bits are equally weighted. For example, a 4-bit binary code converted to a thermometer code would require 15 equally weighted bits (the "all bits off" state does not require an out bit). If the input number was " 8 ", then the thermometer code would have the bottom 8 bits set to "1" and the top 7 bits set to " 0 ". The digital thermometer code needs to be transformed through an encoder into binary data so that the digital decimation filter can remove the out-of-band noise, and give a high-resolution data word at the Nyquist-rate. On the feedback path, a corresponding multi-bit D/A must be in place to convert the digital signal back to analog.

\subsubsection{D/A Element Mismatch}

A well-known drawback of the multi-bit SDM is the nonlinear element mismatch in the multi-bit feedback DAC. Figure 5.8 illustrates an example of the DAC circuit. Normally the multi-bit DAC elements, such as capacitors and resistors, are configured in such a way that a selected number of the elements release their electrical energy into a summing junction that produces an analog output signal equivalent to the digital input. Mismatch error inevitably exists among the elements due to manufacturing variation. Although the absolute error from one element to another can be tightly controlled, the cumulative effect of the mismatched elements can cause significant nonlinear distortion. 
The element mismatch problem does not arise in the 1-bit case because perfect linearity is guaranteed by virtue of the fact that only a single element is used to convert the digital signal to analog.

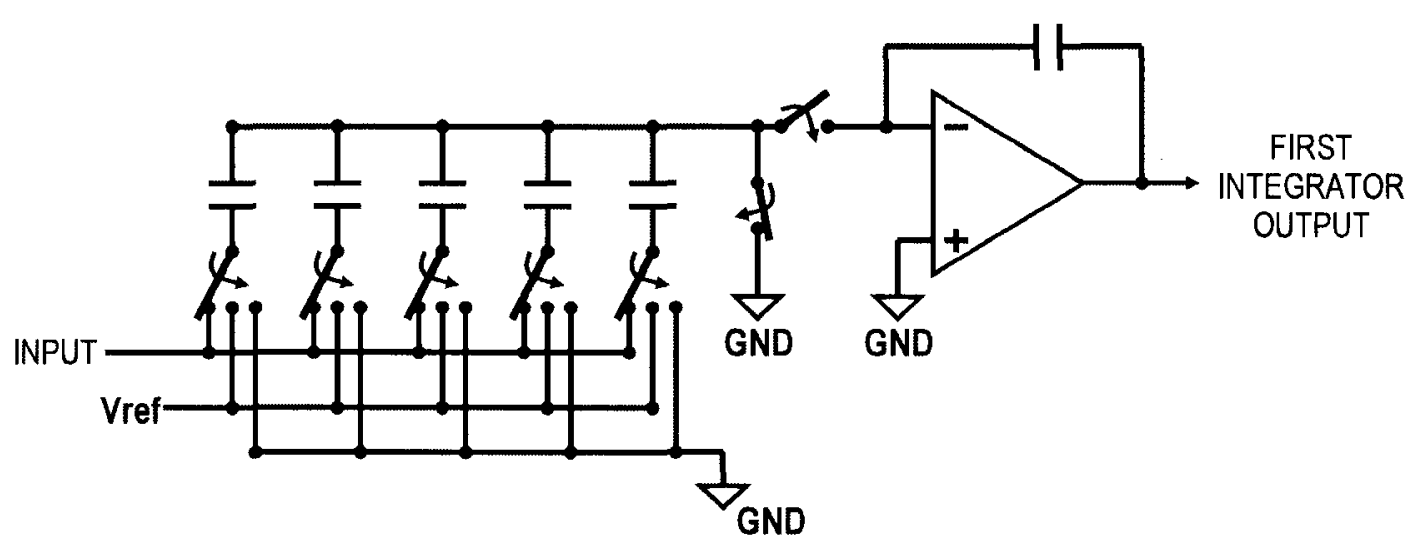

Fig. 5.8 A 5-level D/A Converter Incorporated in The First Integrator of a SDM

The major impact of any element mismatch is an increased noise floor. This is due to the random nature of the modulator output (as discussed in Section 5.2) which randomizes the mismatch error and spreads it to resemble white noise. However, this noise, arising from the feedback path, is not noise-shaped, and therefore needs to be handled carefully.

\subsubsection{Dynamic Element Matching (DEM)}

If the noise contribution from the $\mathrm{D} / \mathrm{A}$ nonlinear element mismatch error becomes significant with respect to system noise, a technique called dynamic element matching (DEM) can be employed. It should be noted that DEM, as a D/A linearization technique, is not simply about randomizing the element selection and spreading nonlinear spurs into white noise. The DEM is also about shaping the mismatch noise on the feedback path in the same way as the noise-shaping is performed in the whole SDM loop, so that the inband noise contribution from the D/A mismatch is suppressed. This is achieved by 
adding a noise-shaped, random element-select unit between the N-level D/A converter and the N-level quantizer, as shown in Figure 5.9.

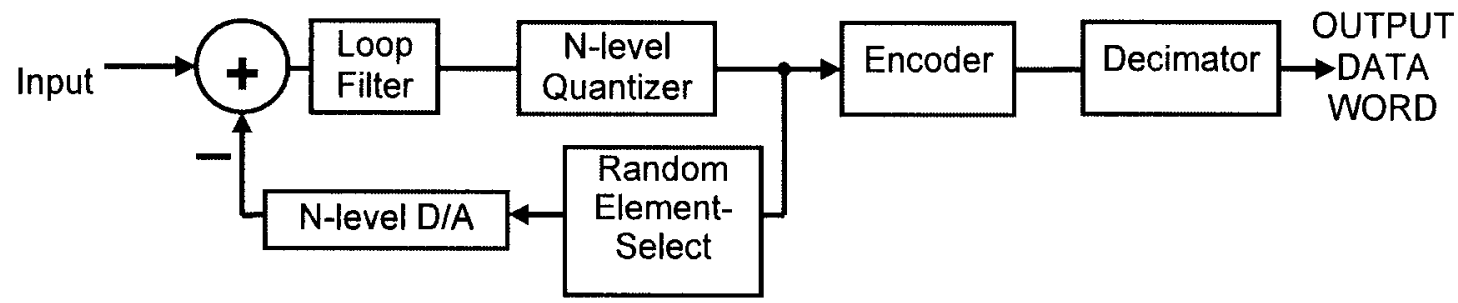

Fig. 5.9 A Multi-bit SDM with Dynamic Element Matching (DEM).

For example, a digital sigma-delta modulator can be used as the random element-select unit and gives rise to noise-shaping at the output of the DAC. This technique of using a digital sigma-delta modulator for noise-shaping is well-known in fractional-N PLL technology. A popular method of implementing the DEM is known as data-weighted averaging (DWA) [21][57][52].

Signal-dependent tones are often observed with the DEM [21]. The challenge for the DEM is that there are two noise-shaped random processes in play; one for the whole SDM loop and one for the D/A on the feedback path. The output of the whole loop is taken as the input of the latter. The two processes should be uncorrelated, otherwise tones may be generated. For this reason, studying a sigma-delta D/A with test tone signals is not adequate.

In addition, there are two implementation issues. First is the added delay in the loop that makes higher-order loops cumbersome [70]. Second, as more DEM circuits are added, caution must be taken not to contribute too much thermal noise and/or clock jitter to the feedback path. 


\subsection{A Quantitative Comparison of 1-Bit and Multi-Bit SDM}

Mismatch error in the D/A of a multi-bit SDM is unavoidable in practice. A quantitative understanding of its impact would be useful, by comparing it with a 1-bit SDM that has no mismatch error. A reasonable comparison should be the signal-to-noise ratio (SNR), and dynamic range that is determined by the minimum and maximum operational signal levels. The comparison must take the bandwidth and noise floor into account, since any dynamic range gained downwards is ultimately limited by the noise floor.

The noise floor can be estimated as follows: A 50-ohm radio communication system at a temperature of $290 \mathrm{~K}$ gives a noise voltage (rms) of $0.9 \mathrm{nV} / \sqrt{\mathrm{Hz}}$ at the frontend. Assuming a $6 \mathrm{~dB}$ noise figure from the front-end to the SDM, the noise voltage will increase to $1.8 \mathrm{nV} / \sqrt{\mathrm{Hz}}$ at the SDM. The selection of bandwidth is critical. A narrow bandwidth will favour a 1-bit SDM in the comparison because of lower noise voltage. A wide bandwidth, on the other hand, will give an advantage to a multi-bit SDM, as shown earlier in Figure 5.7. As a medium of various existing radio systems, a bandwidth of 500 and $200 \mathrm{kHz}$ is a reasonable choice for the comparison. For bandwidths of $500 \mathrm{kHz}$ and $200 \mathrm{kHz}$, the final noise voltage (rms) would be $1.3 \mu \mathrm{V}$ and $0.8 \mu \mathrm{V}$, respectively. Three noise voltages $(0.5,1.3$ and $2 \mu \mathrm{V})$ for the $500 \mathrm{kHz}$ and three noise voltages $(0.4,0.8$ and $1.5 \mu \mathrm{V}$ ) for the $200 \mathrm{kHz}$ are simulated in the following study.

Component mismatch in current IC technology is of the order of $0.05 \% \sim 1 \%$ [72]. Mismatch errors of $0.05 \%, 0.1 \%, 0.5 \%$ and $1 \%$ are used for the multi-bit SDM in this study. The simulation was conducted using MatLab Simulink on a sixth-order band-pass SDM with the NTF shown in Figure 5.7. A 3-bit design was chosen for the multi-bit SDM. Table 5.1 summarizes the simulation conditions for the two SDMs. The difference is basically that the 3-bit SDM has a full-gain NTF but with a DAC mismatch, whereas the 1-bit SDM has no mismatch but has a reduced NTF gain.

Figure 5.10 illustrates an example of the output spectrum for the 1-bit SDM and the 3-bit SDM having $0.5 \%$ mismatch, both at their maximum signal level in the presence of $1.3 \mu \mathrm{V}$ noise, for the $500 \mathrm{kHz}$ case. It can be seen that the 3-bit SDM has a flat noise floor (potential pass-band) that is twice a wide as the 1-bit SDM. It also shows that the 3- 
bit SDM has a higher noise floor due to mismatch error, but it also gains more on the upside and improves the overall dynamic range or SNR.

The SNR for all the cases studied are tabulated in Table 5.2 for the $500 \mathrm{kHz}$ case, and in Table 5.3 for the $200 \mathrm{kHz}$ case. Table 5.2 shows that the 3-bit SDM outperforms the 1-bit SDM in all cases except for a noise voltage of $0.5 \mu \mathrm{V}$ and a $1 \%$ DAC mismatch. For example, the 3-bit SDM having a $0.1 \%$ mismatch outperforms the 1-bit SDM by more than $15 \mathrm{~dB}$. Table 5.3 shows that for a bandwidth of $200 \mathrm{kHz}$, the 1-bit SDM gets a few $\mathrm{dB}$ back in the comparison, as expected for a narrower bandwidth. However, the 3bit SDM still outperforms the 1-bit SDM considerably at the DAC mismatch of $0.1 \%$ and $0.05 \%$. For example, the 3-bit SDM having a $0.1 \%$ mismatch outperforms the 1-bit SDM by about $14 \mathrm{~dB}$.
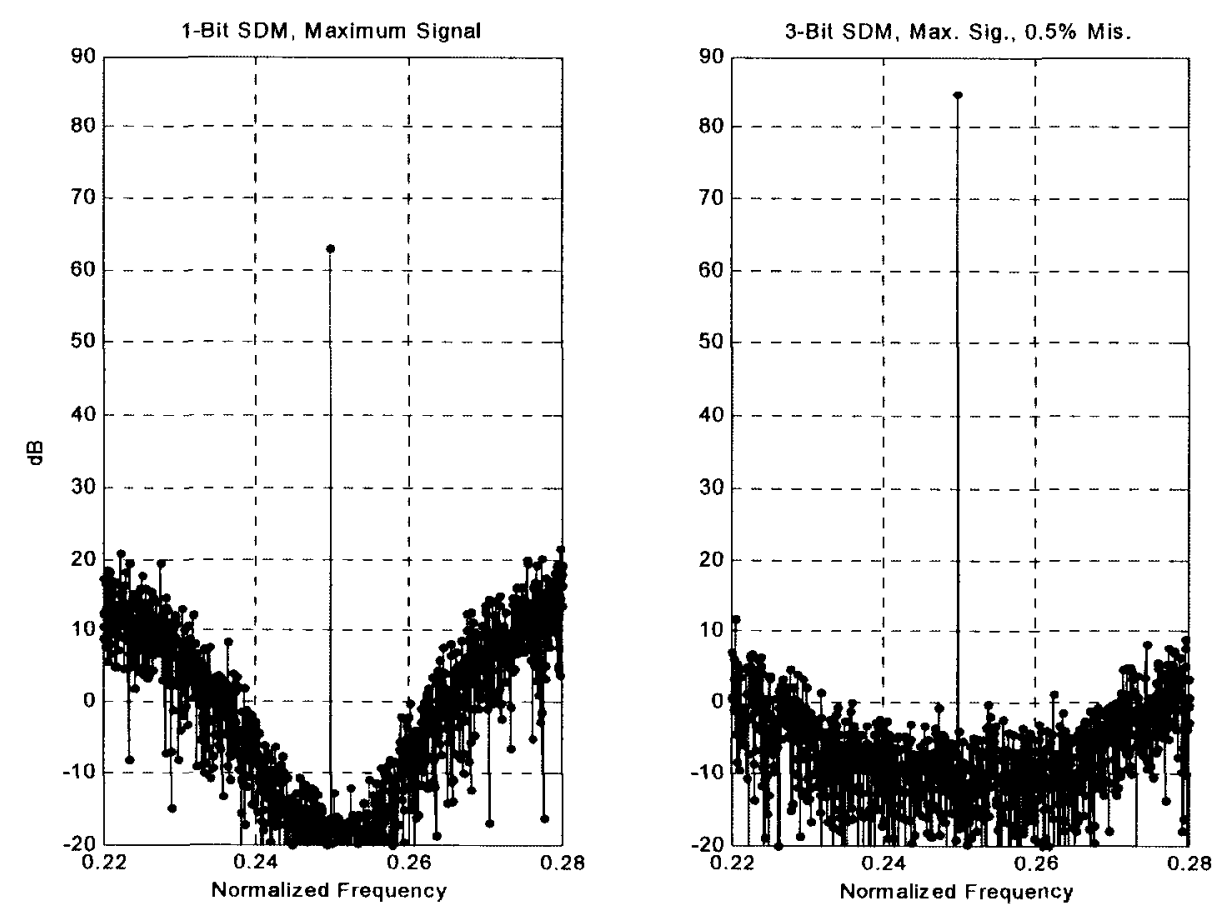

Fig. 5.10 Maximum Signal Output Spectrum of 1-bit SDM vs. 3-bit SDM of 0.5\% Mismatch under $1.3 \mu \mathrm{V}$ Noise Voltage 
Table 5.1. Simulation Conditions for the Two SDMs

\begin{tabular}{|c|c|c|}
\hline & 1-Bit SDM & 3-Bit SDM \\
\hline NTF & $\frac{\left(1+z^{-2}\right)^{3}}{\left(1+0.43 z^{-2}\right)^{3}}$ & $\left(1+z^{-2}\right)^{3}$ \\
\hline & & 1 \\
DAxth-order Bandpass & 0 & 0.5 \\
& & 0.1 \\
& & 0.05 \\
\hline Bandwidth, kHz & 500 & 500 \\
& 200 & 200 \\
\hline Center Freq, MHz & 10 & 10 \\
\hline Sampling Rate, MHz & 40 & 40 \\
\hline Input-Referred Noise & 0.5 & 0.5 \\
Voltage, $\mu \mathrm{V}$ & 1.3 & 1.3 \\
For 500 kHz case & 2 & 2 \\
& & \\
For 200 kHz case & 0.4 & 0.4 \\
& 0.8 & 0.8 \\
& 1.5 & 1.5 \\
\hline
\end{tabular}

Table 5.2. Simulation Results for SNR (signal-to-noise ratio) for $500 \mathrm{kHz}$ Bandwidth

\begin{tabular}{|c|c|c|c|c|}
\hline SDM & DAC & \multicolumn{3}{|c|}{ Input-Referred Noise Voltage } \\
\cline { 3 - 5 } & Mismatch & $0.5 \mu \mathrm{V}$ & $1.3 \mu \mathrm{V}$ & $2 \mu \mathrm{V}$ \\
\hline 1-Bit & $0 \%$ & 63.6 & 60.0 & 58.4 \\
\hline \multirow{3}{*}{$3-B i t$} & $1 \%$ & 61.9 & 62.1 & 62.4 \\
\cline { 2 - 5 } & $0.5 \%$ & 68.6 & 68.3 & 67.4 \\
\cline { 2 - 5 } & $0.1 \%$ & 78.8 & 76.1 & 74.4 \\
\cline { 2 - 5 } & $0.05 \%$ & 80.8 & 77.0 & 75.2 \\
\hline
\end{tabular}

Table 5.3. Simulation Results for SNR for $200 \mathrm{kHz}$ Bandwidth

\begin{tabular}{|c|c|c|c|c|}
\hline SDM & \multirow{2}{*}{$\begin{array}{c}\text { DAC } \\
\text { Mismatch }\end{array}$} & \multicolumn{3}{|c|}{$\begin{array}{c}\text { Input-Referred Noise } \\
\text { Voltage }\end{array}$} \\
\cline { 3 - 5 } & & $0.4 \mathrm{uV}$ & $0.8 \mathrm{uV}$ & $1.5 \mathrm{uV}$ \\
\hline 1-Bit & $0 \%$ & 71.5 & 68.1 & 65.6 \\
\hline \multirow{3}{*}{ 3-Bit } & $1 \%$ & 66.3 & 66.5 & 66.0 \\
\cline { 2 - 5 } & $0.5 \%$ & 71.2 & 72.0 & 71.4 \\
\cline { 2 - 5 } & $0.1 \%$ & 84.1 & 82.3 & 80.0 \\
\cline { 2 - 5 } & $0.05 \%$ & 85.9 & 83.9 & 81.2 \\
\hline
\end{tabular}




\subsection{Multibit Reference Feedback SDM}

The feedback signal in a SDM loop is normally taken from the digital output, D/A converted and sent back to the input of the SDM. This signal flow is shown in Figure 5.11 as a dotted line. There is a discontinuity in terms of the signal level for this kind of arrangement. The digital output is a logic state whose signal level does not need to match the analog input level. However, the signal level needs to be matched in a SDM loop. Therefore the reference levels in the quantizer and D/A need to be carefully set and maintained over process and temperature variations. This can be achieved by employing a high-precision temperature-compensated band-gap reference circuit.

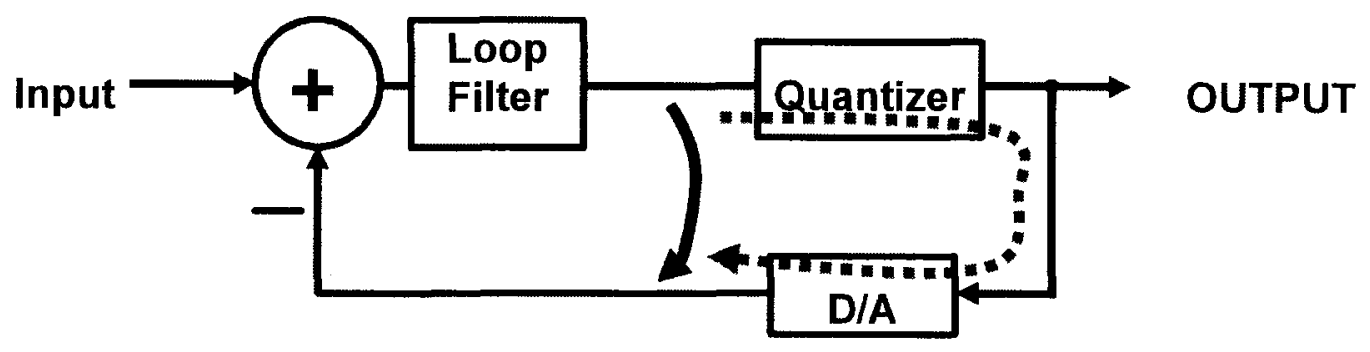

Fig. 5.11 Direct Feedback of the Reference versus Conventional Feedback of the Digital Output

However, another solution for signal matching is a reference feedback as illustrated by the solid line in Figure 5.11. First, examine closely the operation of quantizer. The analog input signal to the quantizer is compared with an array of reference threshold levels; one reference level, often the closest to the input level, is selected and such a state is related to a digital output code. For example, when the input is lower than all the reference levels, the digital output could be an all ' 0 ' state. The digital output code thus represents the analog input with quantization errors, namely $A / D$ conversion. It can be seen that the selected reference is equal to the analog input in signal level with, of course, 
quantization errors. In terms of the signal level, the selected reference is a true representation of the analog input signal, rather than the digital output code. Therefore, feedback of the reference should not change the loop transfer functions. As a matter of fact, doing so brings significant benefits in signal scaling as will be discussed soon. Figure 5.12 is an example implementation for the reference feedback SDM. A switch controlled by the digital output signal is placed in the feedback path to ensure that the correct reference voltage is selected. This can also be viewed as a voltage divider type DAC, which shares the reference voltage generator with the multi-bit quantizer, and as a result, the circuits are much simpler due to the sharing.

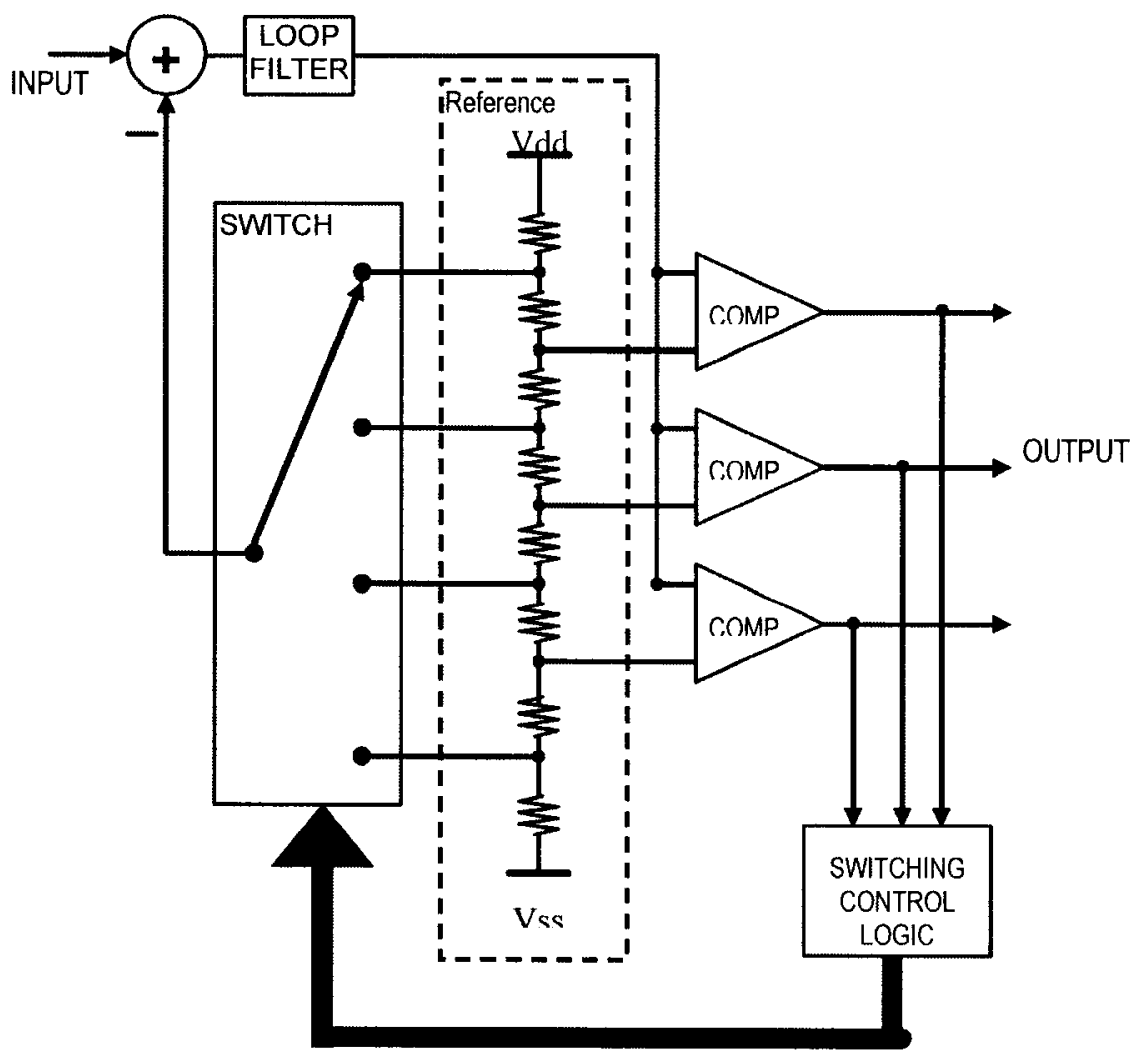

Fig. 5.12 An Example of Reference Feedback SDM 


\subsubsection{Signal Matching}

This kind of reference feedback has been successfully implemented in a SDM circuit [69]. However, there are no further studies on the reference feedback SDM to this date. In this section, we will study signal matching in a sigma-delta modulator in general, and discuss some unique advantages of the reference feedback SDM in terms of reference temperature drift and intentional reference change for dynamic range or AGC control. First, rewrite the SDM transfer function from Eq. (5.5) with the feedback noise term D set to zero for simplicity:

$$
Y=\frac{H}{1+H} X+\frac{1}{1+H} Q
$$

Signal mismatch can be investigated by assuming a loss factor of $k$ in the loop. This is equivalent to replacing the loop filter transfer function $H$ with $H / k$. We can therefore have the following new transfer function from Eq. (5.6):

$$
Y=\frac{H}{k+H} X+\frac{k}{k+H} Q
$$

In the following, we will study a 6th-order bandpass SDM to demonstrate the impact of this signal mismatch in noise-shaping, stability and dynamic range degradation. The 6thorder SDM has a noise transfer function $N T F=\left(1+z^{-2}\right)^{3}$, for which the loop filter transfer function can be obtained as $H=\left(-3 z^{-2}-3 z^{-4}-z^{-6}\right) /\left(1+z^{-2}\right)^{3}$. Substituting this $H$ in Eq. (5.7), we can plot the NTF while varying loss factor $k$, as shown in Figure 5.13. 


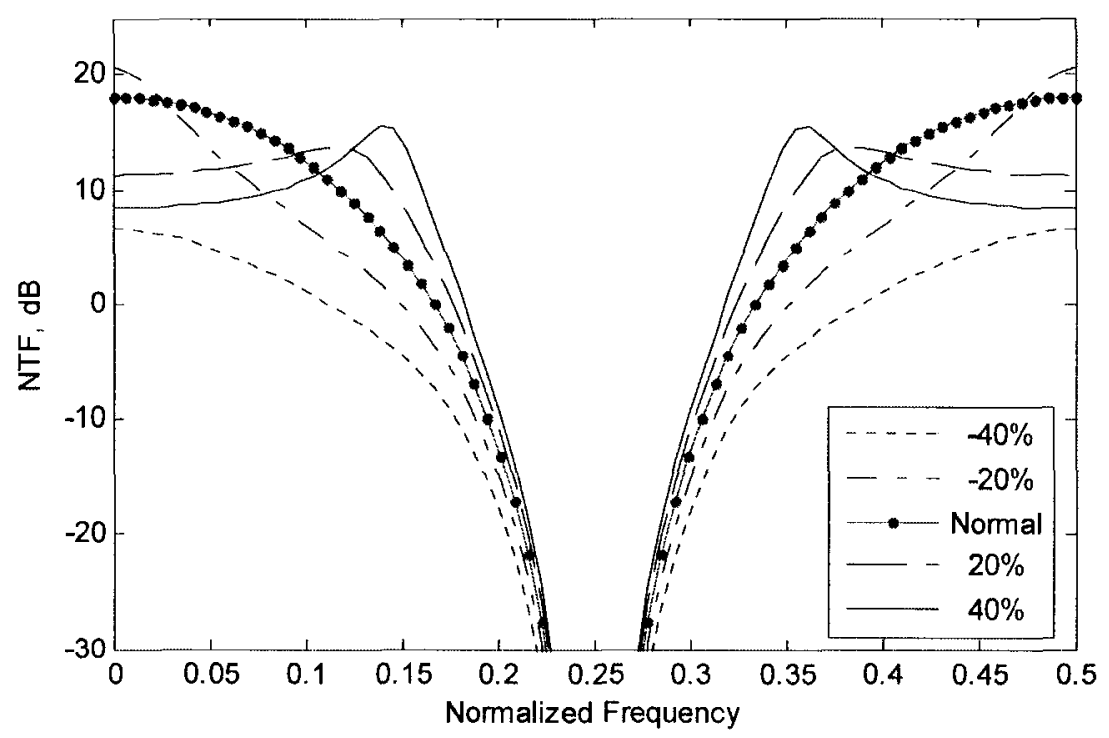

Fig. 5.13 Noise Transfer Function for Different Loss Factor in the Loop

It can be seen from Figure 5.13 that signal mismatch tends to reduce the noise-shaping gain and pushes the noise inward as the loss factor increases, both of which are indicative of deteriorated noise-shaping, and a less stable loop. The direct impact of a less stable loop is reduced dynamic range. This can be observed through time domain simulation. Such a simulation was conducted on MatLab with the quantizer set to 3 bits. The change in the loss factor $\mathrm{k}$ was implemented by varying the reference of the quantizer. For example, increasing the reference level by $20 \%$ represents an increase of the loss factor $k$ by the same amount.

A drop in the maximum input signal level is recorded with respect to the varying reference. In the simulation, the maximum input is considered as the last input level (in steps of $0.1 \mathrm{~dB}$ ) that does not saturate the quantizer. When the quantizer is saturated, a collapsed noise-shaping is observed. The simulation results are plotted in Figure 5.14.

It can be seen from Figure 5.14 that the impact of the signal mismatch is severe when the reference level decreases. A decrease in the reference level (loss factor) means an increase in gain. As a result, the loop is less tolerant of gain variations, because a gain in the loop tends to saturate the quantizer. In the other direction, the maximum input level drops in a linear fashion up to a reference change of $75 \%$, beyond which the loop is practically unstable. 


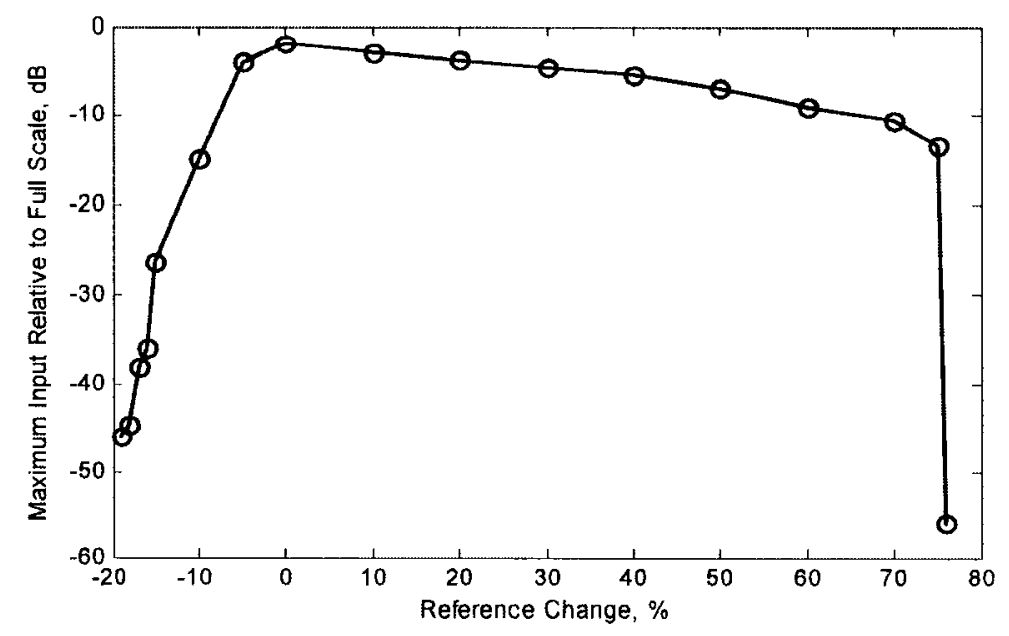

Fig. 5.14 Drop of Maximum Input Level with Changing Reference

Temperature drift is a major source for an undesired reference change. The above analysis on signal matching in a SDM loop explains why a reference circuit needs to be implemented by temperature-compensated band-gap circuits.

Reference change also can be intentional, yielding a large change in dynamic range or $\mathrm{AGC}$ control, as in conventional Nyquist-rate $\mathrm{A} / \mathrm{D}$ conversion. However, this is not an option for conventional sigma-delta modulator. A large amount of reference change will make the SDM loop unstable as shown in Figure 5.14.

It appears from the above analysis that a reference change, either undesired or intentional, is not compatible with conventional SDM because of signal mismatching in the loop. On the other hand, due to its advantage in signal matching, the reference feedback SDM is not only insensitive to reference temperature drift, but also makes intentional reference change an option for sigma-delta modulation. The advantages of this type of naturally matched SDM are discussed as follows.

Temperature Insensitivity: A significant benefit of the reference feedback SDM is its insensitivity to temperature drift in the reference circuit, since any change in the temperature is directly fed back and absorbed in the noise-shaping loop. This means the 
reference feedback SDM does not require a temperature-compensated reference circuit, which can be a considerable advantage in reference circuit design.

Dynamic Range or AGC Control: Another benefit arising from this naturally signalmatched loop is that an intentional change of the reference by a large amount enables dynamic range or $\mathrm{AGC}$ control, a critical function in radio applications. This is not an option in conventional SDM as discussed above. For a conventional Nyquist-rate A/D converter, such a control can be performed in principle, but it is limited in practice. Since a lot of reference levels are allocated in a limited voltage range, there is not much room left in the Nyquist-rate A/D converter to scale the reference level up or down by a large amount.

System-level simulations were conducted on MatLab Simulink to verify the temperature insensitivity. Since the impact of temperature drift in the reference is shown as reference voltage change, the simulation is performed by varying the reference voltage while the noise-shaping spectrum is recorded. The standard sixth-order band-pass 3-bit SDM with NTF $=\left(1+z^{-2}\right)^{3}$ was simulated, for which the loop filter transfer function is $\left(-3 z^{-2}-3 z^{-4}-z^{-6}\right) /\left(1+z^{-2}\right)^{3}$. The transfer function and all the loop components are easily implemented in MatLab Simulink. For comparison, both the new SDM and a conventional SDM were simulated under same conditions.

When the reference voltage is increased from $10 \%$ to $40 \%$, the noise-shaping spectrum for the reference feedback SDM does not change. However, the conventional feedback SDM shows distorted spectral shapes that gradually shift away from what the linear model predicts, indicating a progressively less stable noise-shaping feedback loop. These results are shown in Figure 5.15. 


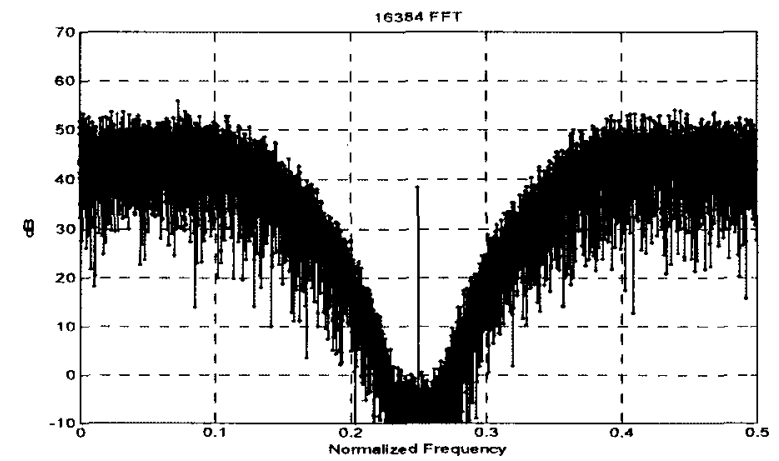

(a)

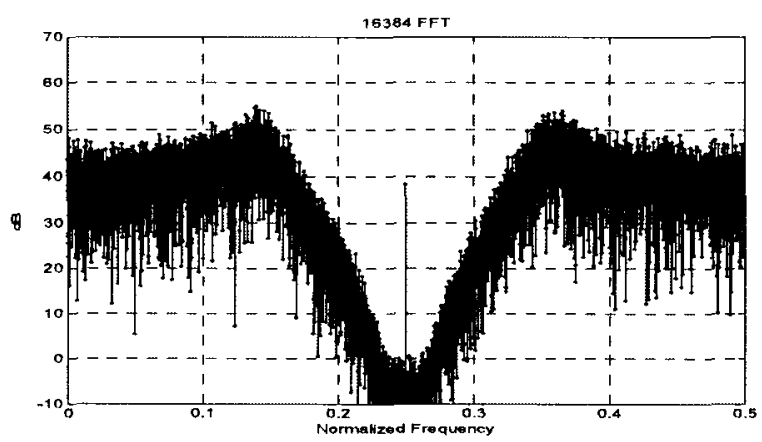

(b)

Fig. 5.15 Noise-Shaping Spectrum for the Conventional SDM with a Reference Voltage Change of (a) $10 \%$ and (b) $40 \%$

\subsubsection{Thermal Noise Consideration}

The summing type DAC in a conventional multi-bit SDM normally employs an operational amplifier to perform the summation, which contributes wideband noise. Such noise in the feedback path cannot be pre-filtered (as can the noise at the SDM input), and will be sampled by the quantizer. In comparison, the reference divider switching type DAC, in which the switch is likely to consist of transmission gate transistors such as CMOS switches, will contribute much less noise to the feedback path of the multi-bit 
SDM. Further, a reference circuit that does not require temperature compensation will use less active components, hence contributing less noise.

\subsection{Summary}

The noise-shaping mechanism of sigma-delta modulation has been reviewed in this chapter. The stability and linearity of a SDM nonlinear feedback loop were discussed in details. In a properly designed SDM, stability and linearity reinforce each other through the feeding back of a noise-like output signal to the quantizer, thereby forcing it operate in a linear manner.

Multibit SDM has a wider bandwidth than its 1-bit counterpart, because a full gain noise-shaping loop can be implemented in the multibit SDM. For 1-bit_SDMs above second-order, any noise-shaping gain has to be reduced to preserve loop stability. A quantitative comparison of the multibit vs the 1-bit SDMs was conducted in some realworld scenario simulations. These show that at moderate element mismatch, a multibit SDM is better than a 1-bit SDM for bandwidths above $500 \mathrm{kHz}$, suggesting that the multibit SDM is a better choice for broadband applications.

The reference feedback multibit sigma-delta modulator was studied in depth for the first time. The study shows that the reference feedback SDM offers a signal-matched noise-shaping loop over process and temperature variations, which is advantageous for reference circuit design and dynamic range \& AGC control. The AGC control by intentional change of the reference level cannot be performed in a conventional feedback SDM and is not practical in a Nyquist-rate A/D converter. It was also shown in this chapter that signal mismatch in a SDM loop causes distorted noise-shaping, and dynamic range or bandwidth loss. 


\section{CHAPTER 6 Design of a Reference Feedback SDM}

The multi-bit reference feedback SDM was introduced in the previous chapter. In this chapter, a $4^{\text {th }}$-order bandpass SDM is designed with a novel cascaded loop filter circuit. Experimental results of the $4^{\text {th }}$-order bandpass SDM will be presented in the next chapter.

A continuous-time (CT) SDM was chosen for the design. There are four advantages for the CT SDM over a discrete time (DT) SDM. First, no sample/hold is required at the SDM input. Sampling takes place at the quantizer within the loop, and sampling errors will be noise-shaped [41]. Second, higher speed operation can be realized. Continuous-time analog filters can operate at a much higher frequency than switched-capacitor filters used in DT SDM. For a switched-capacitor filter, charge transfer in a discrete fashion among capacitors is a limiting factor for the sampling frequency. Third, lower clock feedthrough is achieved, since no clock is employed in the CT loop filter. Finally, anti-aliasing filtering is provided by the CT filter [62]. This is beneficial in the handling of interferers.

DT SDMs are implemented using switched-capacitor (SC) circuits, in which amplifiers with a high gain-bandwidth product (GBW) satisfy the settling requirements. Typically, the GBW is seven times higher than the sampling frequency. CT loop filters do not need clocks. As a result, CT SDMs can potentially operate at a higher clock frequency and/or with less power consumption. In SC circuits, the in-band noise is bounded by the capacitor size. Consequently, CT modulators have smaller size and are less silicon area-consuming than their DT counterparts [74]. Unlike a CT modulator, in a DT modulator large glitches appear on the op-amp RC integrators virtual ground node due to switching transients. Therefore, a CT modulator achieves better linearity performance. Furthermore, when the modulator is integrated in a complete wireless transceiver in CMOS, glitches generated in the DT modulators can potentially couple to 
other critical blocks of the receiver, such as voltage-control oscillators (VCO), LNAs or mixers, and can seriously degrade the receiver sensitivity. Currently, CT modulators are preferred to DT modulators, whatever the application [74].

In a DT modulator, the time constant's variations of the noise-shaping filter achieve excellent matching since they rely on the capacitor ratio. However, this is not the case in CT modulators where the time constant's variation can be between $25 \%$ to $30 \%$ due to resistor and capacitor spreads. This can seriously degrade the SNR performances. Nevertheless, some on-chip biasing techniques that consist of compensating the temperature dependence of hole or electron mobility in silicon, enables the design of accurate time constraints despite process and temperature variations. Another solution widely used for op-amp RC integrator time constant tuning makes use of switchable capacitor arrays. In this case, a calibrator is used to measure the fabricated RC product with a reference clock frequency. From this, a digital code word is generated, which is used to select elements in programmable arrays of capacitors that form the tuning elements of the filter integrators. Both solutions are robust and do not introduce too much circuit complexity. [74]

A bandpass SDM was chosen for the design due to our interest in radio applications. A bandpass sigma-delta modulator (SDM) can be made by choosing a bandpass type (resonator) loop filter, which gives rise to a band-reject type noise transfer function. This is very attractive for radio applications as some IF analog filtering and down conversion can be removed [32][33][34][42], and can be replaced with digital filtering. Analog IF filters generally have poor phase performance, introducing intersymbol interference, whereas digital filters can have a perfectly linear phase response.

The analysis of a CT SDM is more difficult than its DT counterpart, since the noise-shaping loop is a hybrid system. Computer time-domain simulations, which are critical in designing a SDM, run discretely. Therefore, it makes more sense to treat the loop as a discrete system. This can be done by transforming the CT loop filter into an equivalent discrete-time filter. An existing technique, the impulse invariant 
transformation (IIT) [61] as will be explained in the next section, can be employed in this transformation, and in the design of the loop filter [5][19].

\subsection{Loop Filter Transfer Function for CT SDM}

Although the CT SDM is made with a CT analog loop filter, the output of the analog filter is sampled nonetheless at the quantizer, as illustrated in Figure 6.1. Therefore, as seen at the quantizer, the overall loop response can be considered as discrete time. In order for the CT SDM to perform noise-shaping, the sampled response seen at the quantizer needs to be the same as that of a discrete-time (DT) SDM.

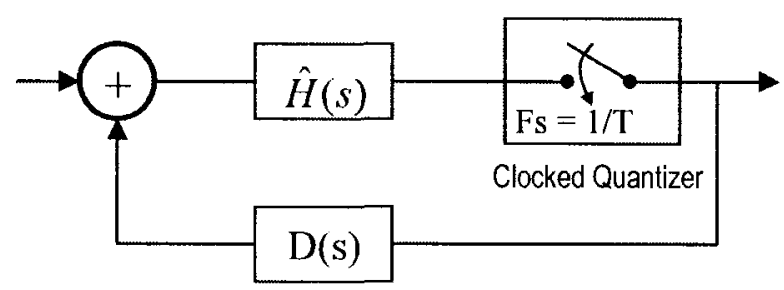

Fig. 6.1 Continuous-Time (CT) Sigma-Delta Modulator

From Figure 6.1, the open loop transfer function from the DAC to the loop filter is

$$
H(s)=\hat{H}(s) D(s)
$$

where $D(s)$ is the DAC transfer function. For zero-order-hold DAC, the impulse response is shown in Figure 6.2, where $0 \leq \mathrm{T}_{1}<\mathrm{T}_{2} \leq \mathrm{T}$ : 


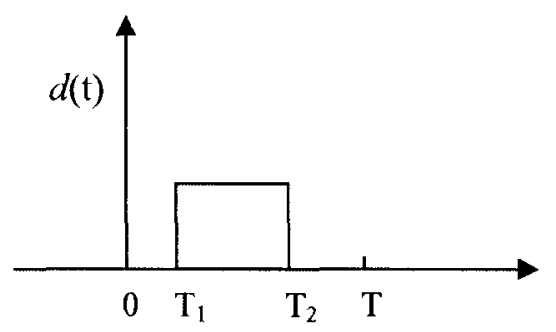

Fig. 6.2 Zero-Order-Hold DAC Impulse Response

$$
D(s)=\frac{e^{-S T_{1}}-e^{-S T_{2}}}{s}
$$

The impulse response of Eq. (6.1) can be obtained as $h(t)=L^{-1}[\hat{H}(s) D(s)]$, where $L^{-1}$ denotes inverse Laplace transformation. Therefore the discrete-time impulse response will be

$$
\begin{aligned}
h(n T) & =\left\{L^{-1}[\hat{H}(s) D(s)]\right\}_{t=n T} \\
& =\left\{\int \hat{h}(t-\tau) d(t) d \tau\right\}_{t=n T}
\end{aligned}
$$

The $\mathrm{z}$-domain representation can be obtained as follows:

$$
\begin{aligned}
H(z) & =Z\left\{L^{-1}[\hat{H}(s) D(s)]\right\}_{t=n T} \\
& =Z\left\{\int \hat{h}(t-\tau) d(t) d \tau\right\}_{t=n T}
\end{aligned}
$$

Since $d(t)$ has a pulse waveform, the above equations are known as the pulse invariant transformation [5][61][62]. For the popular $4^{\text {th }}$ order discrete-time bandpass SDM, the 
NTF (noise transfer function) is $\left(1+\mathrm{Z}^{-2}\right)^{2}$ as illustrated in Figure 6.3. From this the loop filter transfer function can be obtained as:

$$
H(z)=\frac{2 z^{-1}+z^{-3}}{\left(1+z^{-2}\right)^{2}}
$$

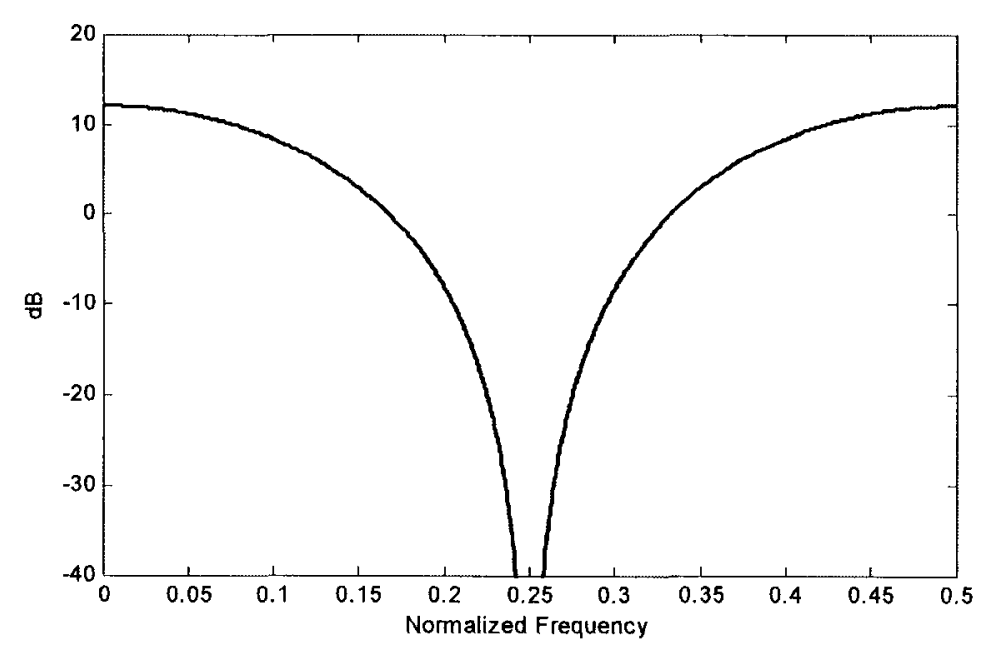

Fig. 6.3 Full-Gain NTF $=\left(1+\mathrm{z}^{-2}\right)^{2}$ of a $4^{\text {th }}$-order Bandpass SDM

For a non-return-to-zero (NRZ) DAC having $T 1=0$ and $T 2=T$, the corresponding CT loop filter has the following transfer function [63]:

$$
\hat{H}(s)=\frac{\left(1-\frac{1}{2 \pi}\right)\left(\frac{\pi}{2 T}\right) s^{3}+\left(0.75+\frac{1}{\pi}\right)\left(\frac{\pi}{2 T}\right)^{2} s^{2}+\left(1+\frac{1}{2 \pi}\right)\left(\frac{\pi}{2 T}\right)^{3} s+0.75\left(\frac{\pi}{2 T}\right)^{4}}{\left[s^{2}+\left(\frac{\pi}{2 T}\right)^{2}\right]^{2}}
$$

Also, for a quarter-delayed return-to-zero (QRZ) DAC having $T 1=T / 4$ and $T 2=3 T / 4$, the CT loop filter has the following transfer function (APPENDIX B): 


$$
\hat{H}(s)=\frac{1.602\left(\frac{\pi}{2 T}\right) s^{3}+1.93\left(\frac{\pi}{2 T}\right)^{2} s^{2}+2.19\left(\frac{\pi}{2 T}\right)^{3} s+1.34\left(\frac{\pi}{2 T}\right)^{4}}{\left[s^{2}+\left(\frac{\pi}{2 T}\right)^{2}\right]^{2}}
$$

The transfer functions of Eq. (6.6) and Eq. (6.7) are derived with a digital delay assigned to the feedback path. This type of continuous-time loop filter transfer function can in general be represented by:

$$
\hat{H}(s)=G \frac{s^{2 N-1}+b_{1} s^{2 N-2}+\cdots+b_{2 N-1}}{\left(s^{2}+a_{1} s+a_{2}\right)^{N}}
$$

where all the coefficients are positive. This has been verified by the author for up to $8^{\text {th }}$ order loops.

The transfer function (6.8) is normally implemented by a circuit composed of multiple inter-connected resonators [62][63][64]. Multiple tuning components in the circuit are used to adjust the coefficients. Such an implementation of the transfer function, as one large circuit, is complicated in design and prototyping, especially for high-order loops.

\subsection{A Novel Cascaded Loop Filter}

The above transfer function can obviously be broken down into cascading sections. As an example, the $4^{\text {th }}$ order transfer function can be represented as:

$$
H(s)=G \frac{s^{2}+b s+c}{s^{2}+a_{1} s+a_{2}} \cdot \frac{s+d}{s^{2}+a_{1} s+a_{2}}
$$


where all the coefficients are still positive. The first section can be implemented as a Fleischer-Tow biquad circuit [65]. However, no existing biquad circuitry has been found for the second section. A new biquad circuit was therefore conceived, and its singleended version is shown in Figure 6.4.

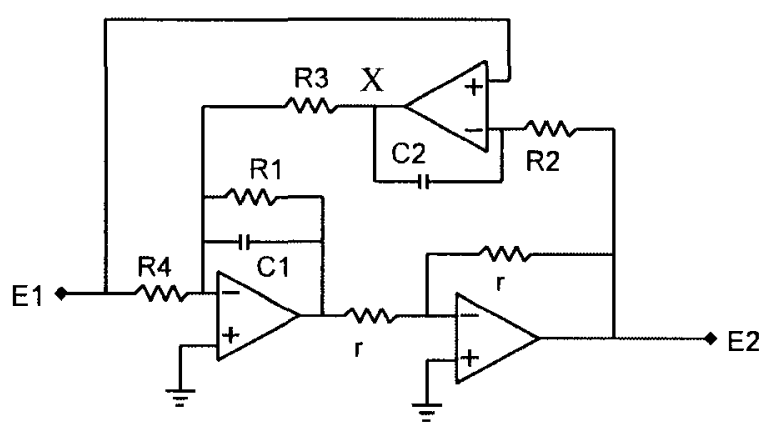

Fig. 6.4 A New Biquad Circuit for CT SDM Loop Filter

The transfer function of this new biquad circuit can be derived as follows

$$
\begin{aligned}
& \frac{E_{1}}{R_{4}}-E_{2}\left(s C_{1}+1 / R_{1}\right)+\frac{X}{R_{3}}=0 \\
& \left(X-E_{1}\right) s C_{2}+\frac{E_{2}-E_{1}}{R_{2}}=0 \quad \Rightarrow X=E_{1}+\frac{E_{1}}{s C_{2} R_{2}}-\frac{E_{2}}{s C_{2} R_{2}}
\end{aligned}
$$

Substituting $X$ into the first equation, we have

$$
\frac{E_{1}}{R_{4}}-E_{2}\left(s C_{1}+1 / R_{1}\right)+\frac{E_{1}}{R_{3}}+\frac{E_{1}}{s C_{2} R_{2} R_{3}}-\frac{E_{2}}{s C_{2} R_{2} R_{3}}=0
$$

or

$$
E_{1}\left[s C_{2} R_{2}\left(\frac{R_{3}}{R_{4}}+1\right)+1\right]=E_{2}\left(s^{2} C_{1} C_{2} R_{2} R_{3}+s C_{2} R_{2} R_{3} / R_{1}+1\right)
$$

Finally 


$$
H(s)=\frac{s C_{2} R_{2}\left(1+R_{3} / R_{4}\right)+1}{s^{2} C_{1} C_{2} R_{2} R_{3}+s C_{2} R_{2} R_{3} / R_{1}+1}
$$

It can be seen that the new biquad does have the needed transfer characteristics. By cascading it with existing biquads, the new biquad allows one to make any higher order transfer function, significantly simplifying high-order loop filter design and implementation.

\subsection{Design of the $4^{\text {th }}$-order CT Loop Filter}

The final $4^{\text {th }}$-order CT loop filter was chosen from (6.6), which can be rewritten as follows:

$$
\begin{aligned}
H(S) & =0.841 \frac{S^{3}+1.27052 S^{2}+1.37856 S+0.89196}{\left(S^{2}+1\right)^{2}} \\
& \approx 0.84 \frac{\left(S^{2}+0.4 S+1\right)(S+0.87)}{\left(S^{2}+1\right)^{2}}
\end{aligned}
$$

where $S$ is normalized to the resonant frequency, namely $S=s 2 T / \pi$. Eq. (6.12) is an ideal transfer function with infinite $Q$. In a real circuit, $Q$ is finite due to inevitable circuit losses. Therefore, the realizable filter will have the following transfer function:

$$
\begin{aligned}
H(S) & =0.84 \frac{\left(S^{2}+0.4 S+1\right)(S+0.87)}{\left(S^{2}+0.017 S+1\right)^{2}} \\
& =0.84 \frac{S^{2}+0.4 S+1}{S^{2}+0.017 S+1} \cdot \frac{S+0.87}{S^{2}+0.017 S+1}
\end{aligned}
$$


The first section of the filter can be implemented as a Fleischer-Tow biquad [65], and the second by the new biquad circuit presented in the previous section. Such a circuit has been designed and is shown in Figure 6.5. The operational amplifier used is a Texas Instruments THS4211. Circuit simulation with a SPICE-modeled THS4211 was conducted on an ADS circuit simulator. The circuit was then prototyped. Figure 6.6 shows the simulation results along with the measurement result.

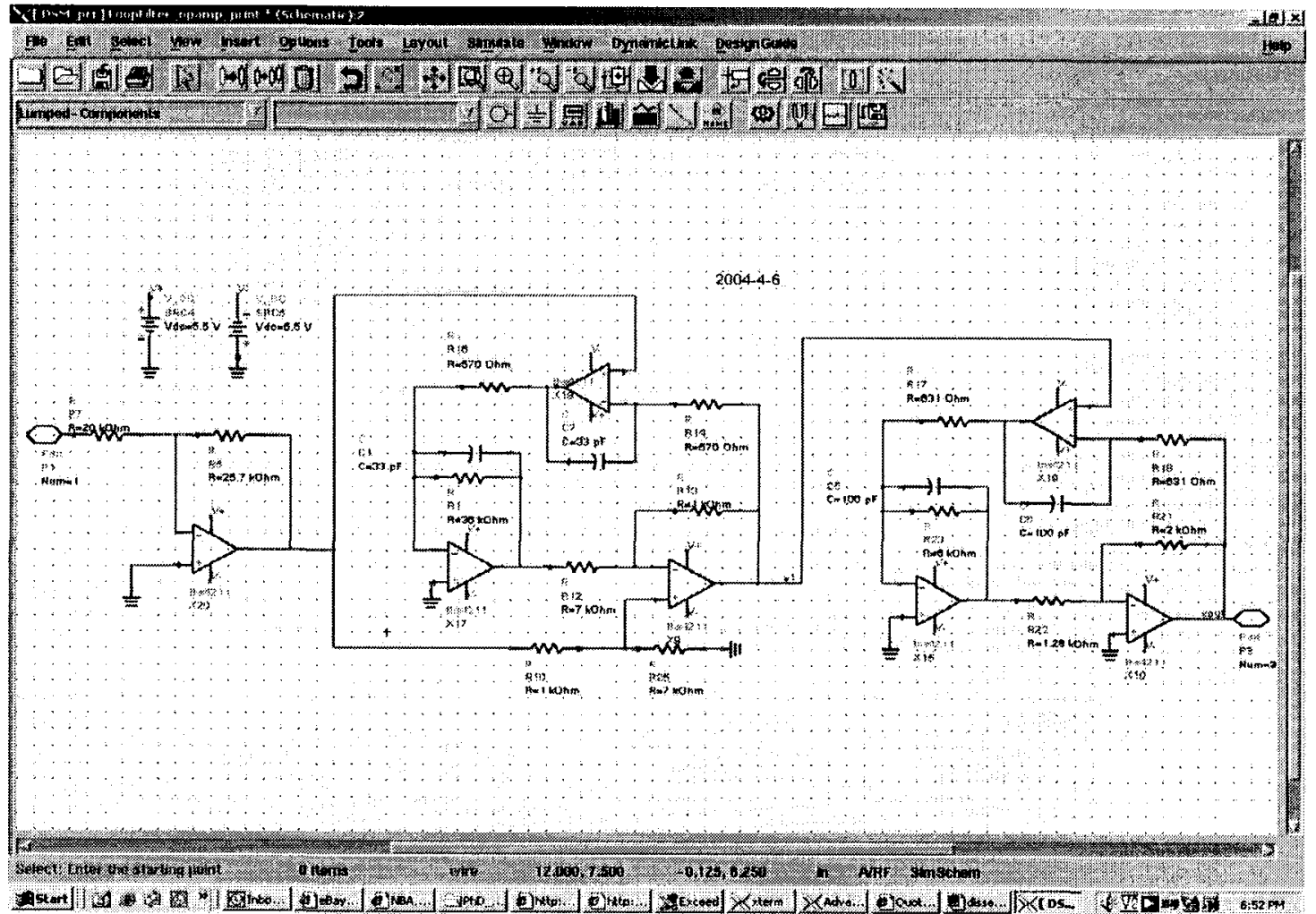

Fig. 6.5 Cascaded Loop Filter with the New Biquad

The center frequency of the filter, and hence that of the SDM, was designed as 3.125 $\mathrm{MHz}$, with the sampling clock being $12.5 \mathrm{MHz}$. These choices were made considering the gain-bandwidth of the available components. 


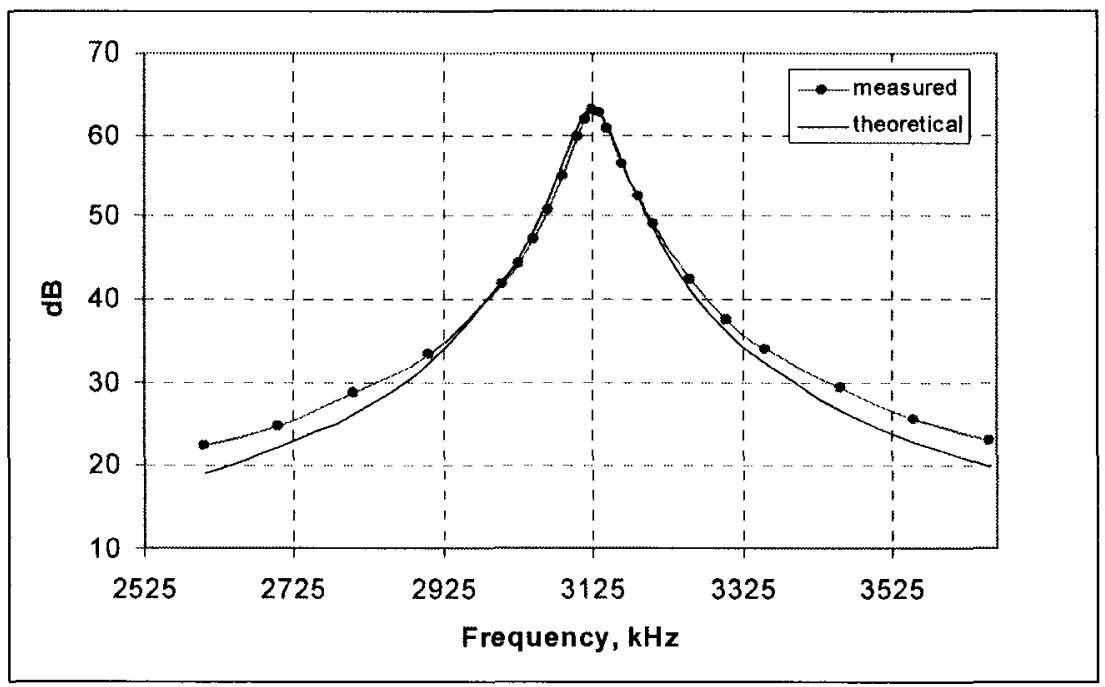

Fig. 6.6 Loop Filter Simulation and Measurement

\subsection{Transmission Gate Switch}

The designed reference feedback SDM is based on Figure 5.12. The multi-throw switch in Figure 5.12 can be implemented using transmission gate switches, for which commercial products are available. The transmission gates contribute much less noise than an operational amplifier in a conventional SDM.

A simple switching control circuit was designed as shown in Figure 6.8. The thermometer code is taken as the input, and the parallel XOR gates ensure only the right switch arm is selected. 


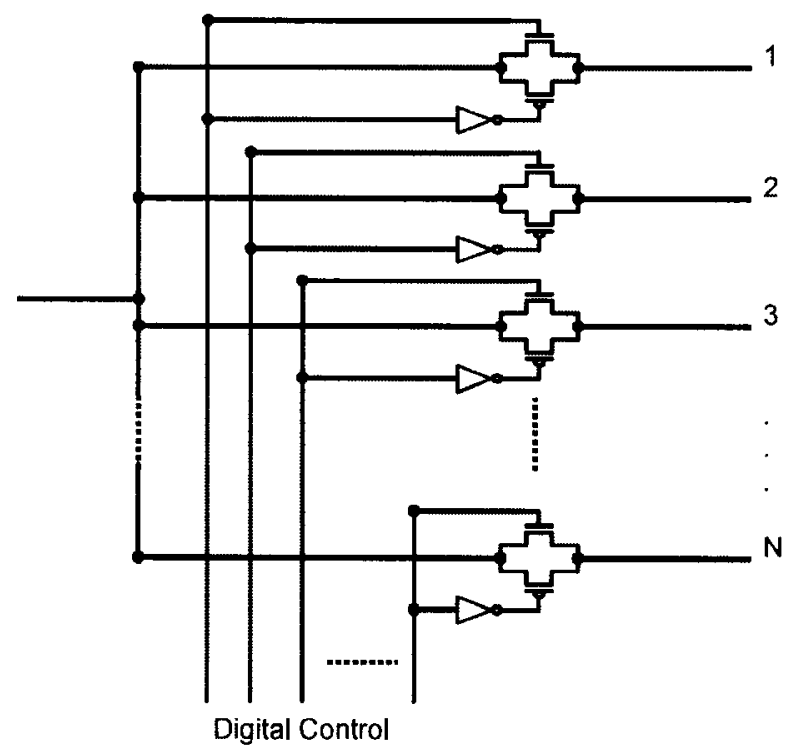

Fig. 6.7 A Switch Matrix of CMOS Gates for The Reference Feedback SDM

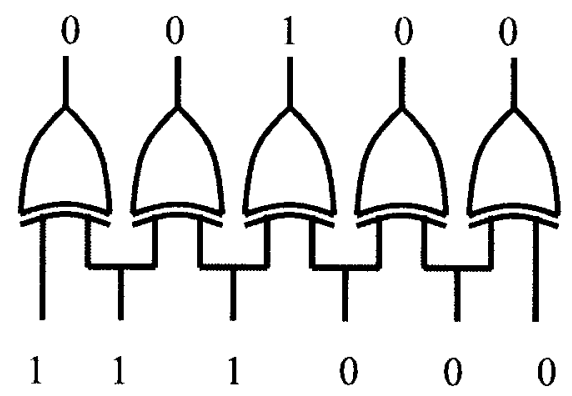

Fig, 6.8 A Simple XOR Gates for Switch Control 


\subsection{Circuit Simulation}

Circuit simulation for this particular design was conducted on the ADS circuit simulator, a critical step to establish how the circuit functions, before prototyping. The simulation set-up is shown in Figure 6.9. The loop filter block is the SPICE-modeled filter in Figure 6.5. The multi-throw switch and the quantizer are realized as ideal circuits. The simulation was successful. Figure 6.10 shows an example of the results.

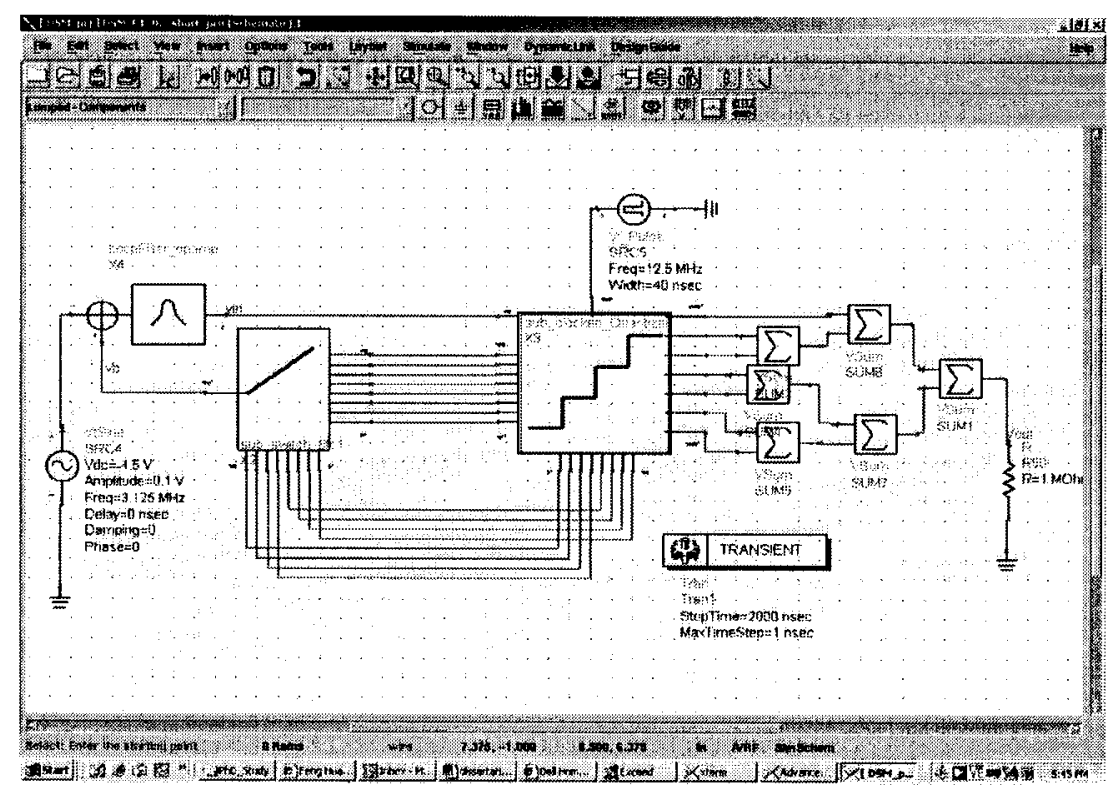

Fig. 6.9 Simulated Circuit for the New SDM

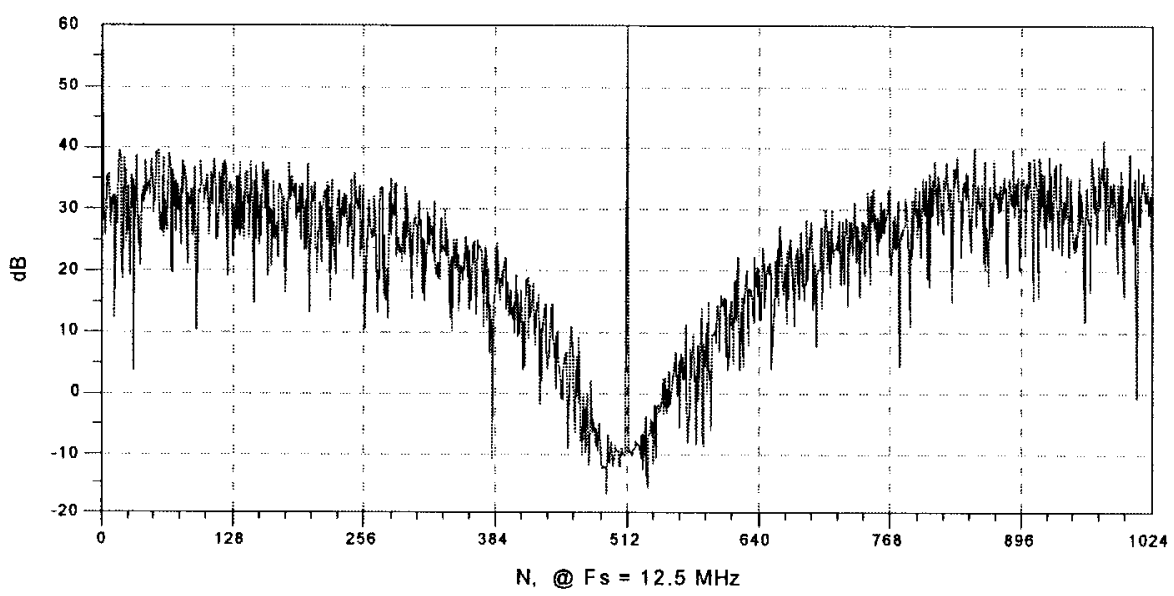

Fig. 6.10 One of the Circuit Simulation Results 


\subsection{Summary}

A fourth order 3-bit CT bandpass reference feedback SDM was designed, whose CT loop filter transfer function can be derived from its DT counterpart by a pulse invariant transformation. Cascaded biquad sections were used to implement the CT filter, and a novel biquad circuit was developed for this implementation. The novel biquad circuit can allow any higher order CT loop filter to be designed and realized by simply cascading regular biquad circuits. The $4^{\text {th }}$-order CT loop filter with the novel biquad was built and tested. Circuit simulation for the 3-bit CT reference feedback SDM was also successfully conducted, using SPICE models of real analog components. 


\section{CHAPTER 7 Experimental Prototype of the Designed SDM}

An experimental prototype was constructed to verify the reference feedback SDM introduced in Chapter 5 , along with the new cascaded biquad loop filter presented in Chapter 6. Results from the theoretical analysis and simulations in Section 5.5 and 5.6 will also be examined in this chapter. A circuit board approach was chosen for the experiment for two reasons; easy engineering prototyping, and low cost. A limitation to this approach is the frequency constraint of available discrete components. Because of this, a clock rate of $12.5 \mathrm{MHz}$ was chosen, and the signal passband was centered at 3.125 MHz.

\subsection{Circuit Board Prototyping}

A 3-bit reference feedback SDM was implemented with the following components.

Table 7.1 Components Used for the 3-bit Reference Feedback SDM

\begin{tabular}{|c|c|c|c|c|}
\hline Component & Part Number & $\begin{array}{c}\text { Number of Parts } \\
\text { on the Prototype }\end{array}$ & Vendor & Notes \\
\hline Comparator Array & MAX900 & 2 & Maxim & 8 ns delay \\
\hline CMOS Switches & MAX4522 & 2 & Maxim & 40 ns delay \\
\hline XOR Array & SN74HC86 & 2 & TI & 10 ns delay \\
\hline Op-Amp & THS4211 & 8 & TI & \\
\hline Digital Buffer & SN74HC541 & 2 & TI & 10 ns delay \\
\hline Digital Buffer & SN74HC240 & 1 & TI & 10 ns delay \\
\hline
\end{tabular}


Figure 7.1 shows the CAD layout of the circuit board. As a reference, a summing-type DAC is also placed on the board. The same resistor elements were used in the DAC as those in the quantizer reference divider.

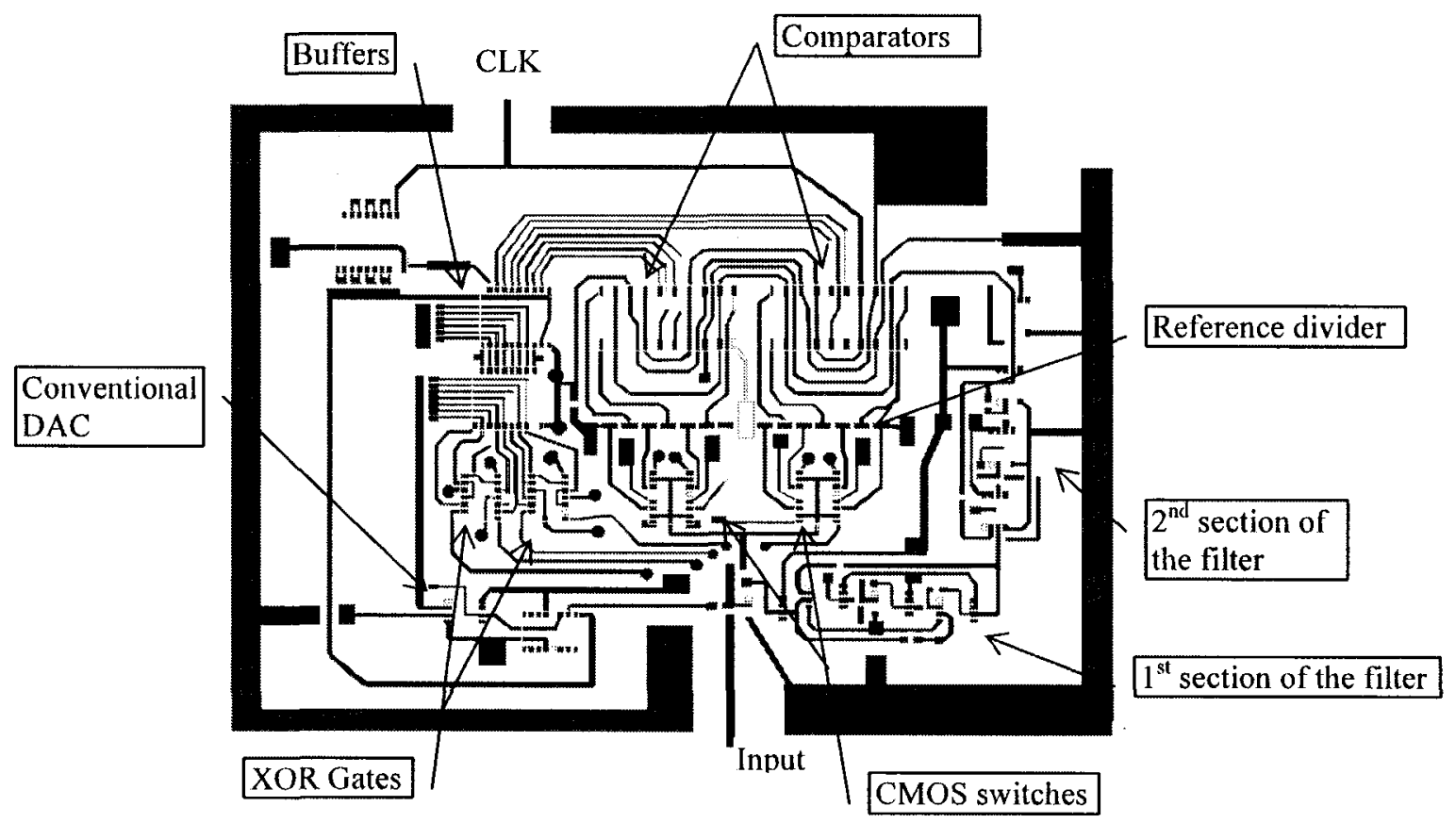

Fig. 7.1 Circuit Board Layout

\subsection{Test Set-up}

The summed output from the thermometer code of the SDM prototype was over-sampled at $100 \mathrm{MHz}$ and stored using a digital oscilloscope. The data were then time-recovered and decimated down to $12.5 \mathrm{MHz}$ and logically translated into digital data, whose spectrum was finally obtained by FFT. The test set-up is illustrated in Figure 7.2. 


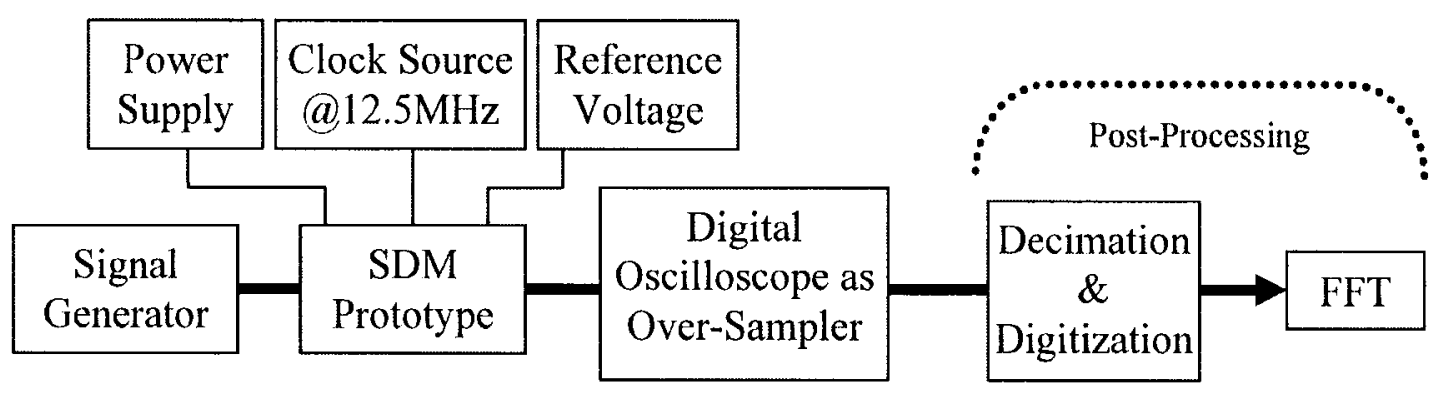

Fig. 7.2 Test Set-up

\subsection{Results and Discussions}

Figure 7.3 is a snapshot of the SDM output waveform captured by the digital oscilloscope. The bottom trace is the $12.5 \mathrm{MHz}$ clock. The top trace shows that the quantized output signal pulses stay at a set of voltage levels, ready for digitization.

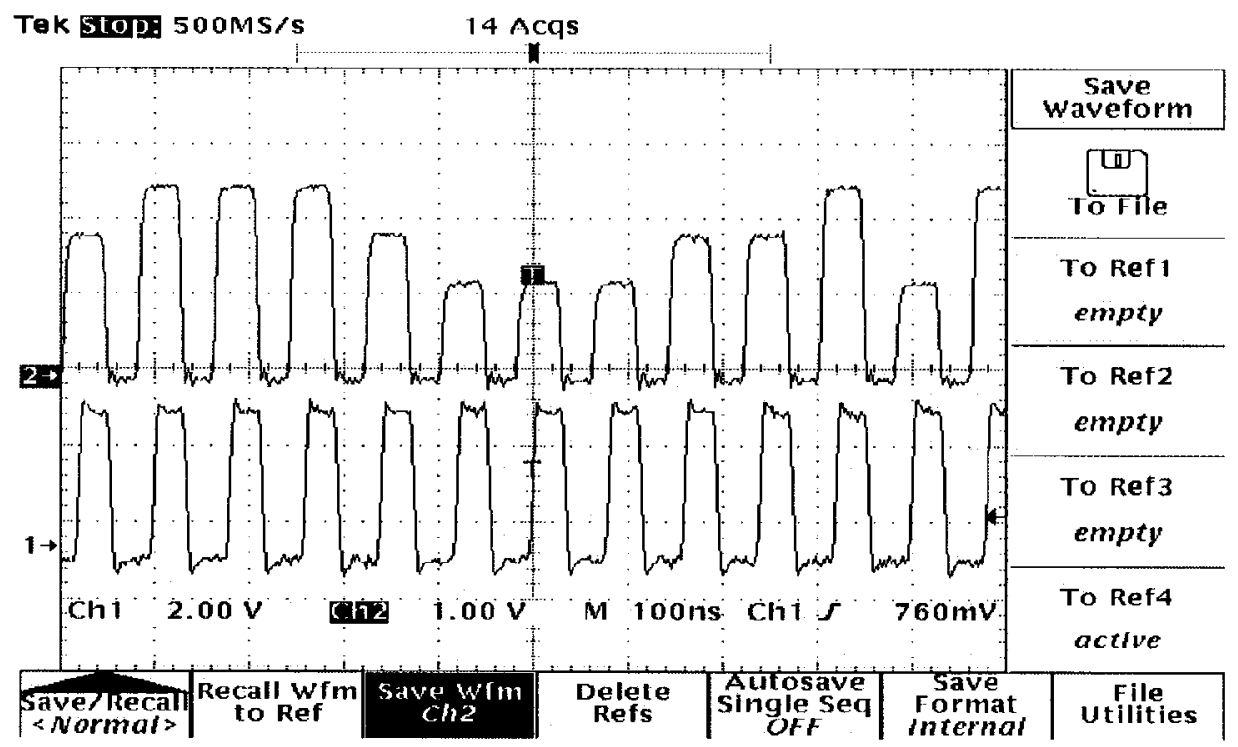

Fig. 7.3 Snapshot of The SDM Output Waveform (Top); Bottom Trace is The Clock 
An example of the digitized output pulses is shown in Figure 7.4. The rate of the digital data at this point is $100 \mathrm{MHz}$, which will be further decimated to $12.5 \mathrm{MHz}$ for the final FFT,

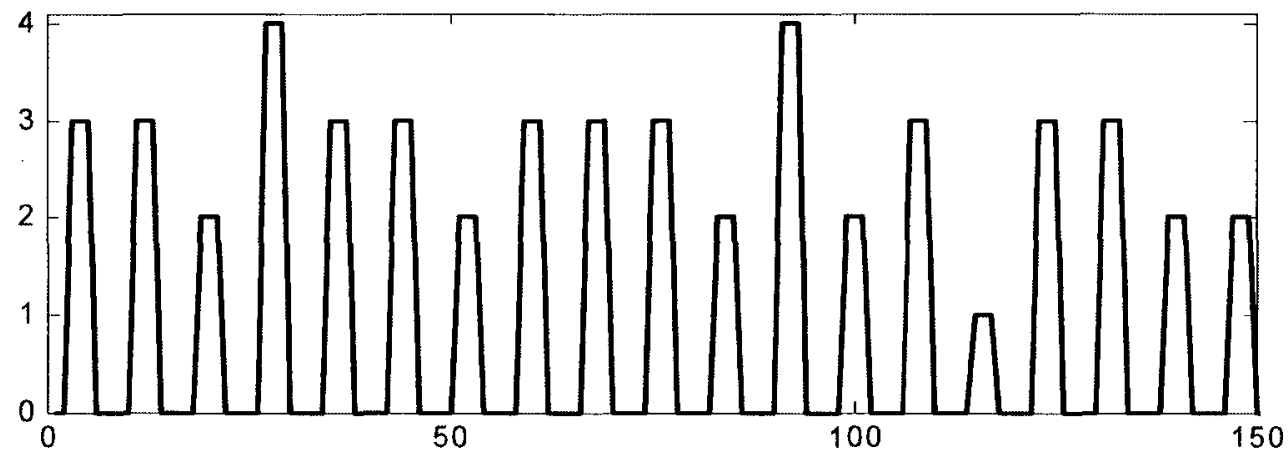

Fig. 7.4 An Example of the Digitized Data (100MHz) prior to Decimation

Figures 7.5 through 7.7 are a few examples of the SDM output spectrum performed by a 4096-point FFT.

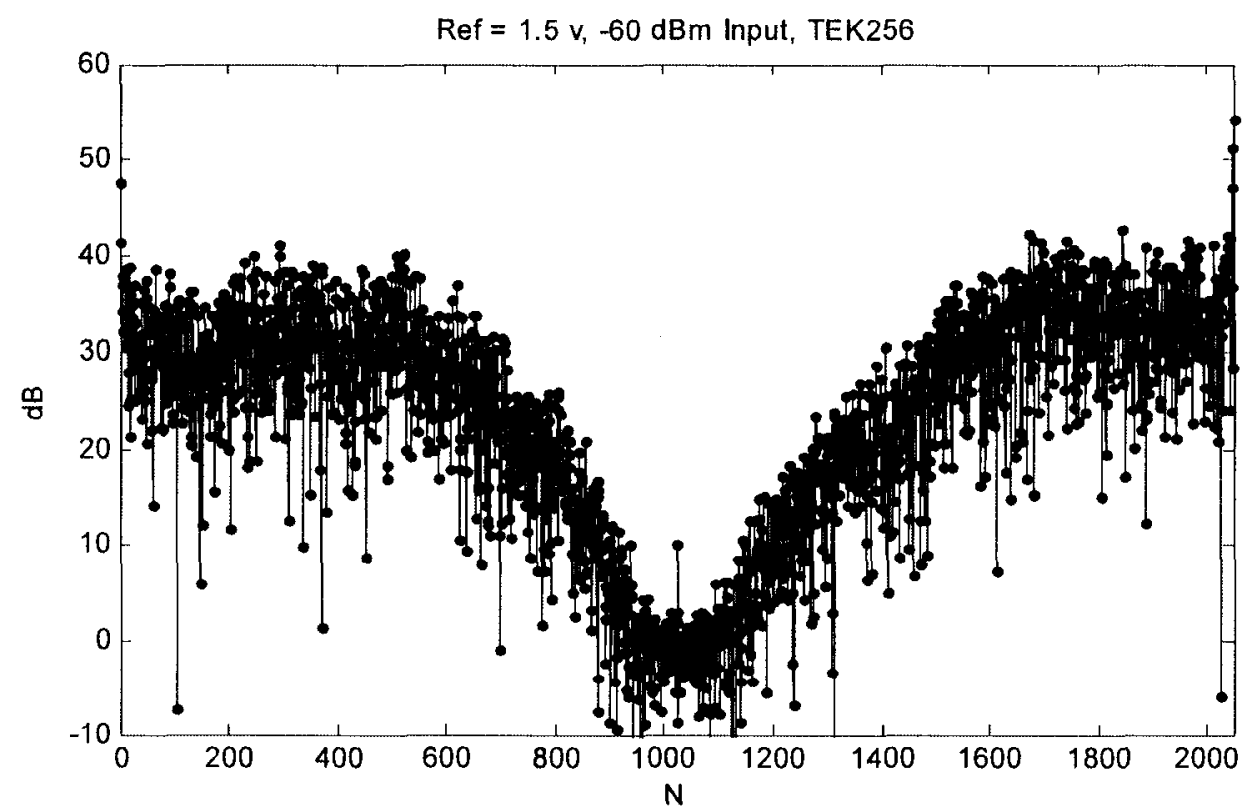

Fig. 7.5 Output Spectrum of a $-60 \mathrm{dBm}$ Input 


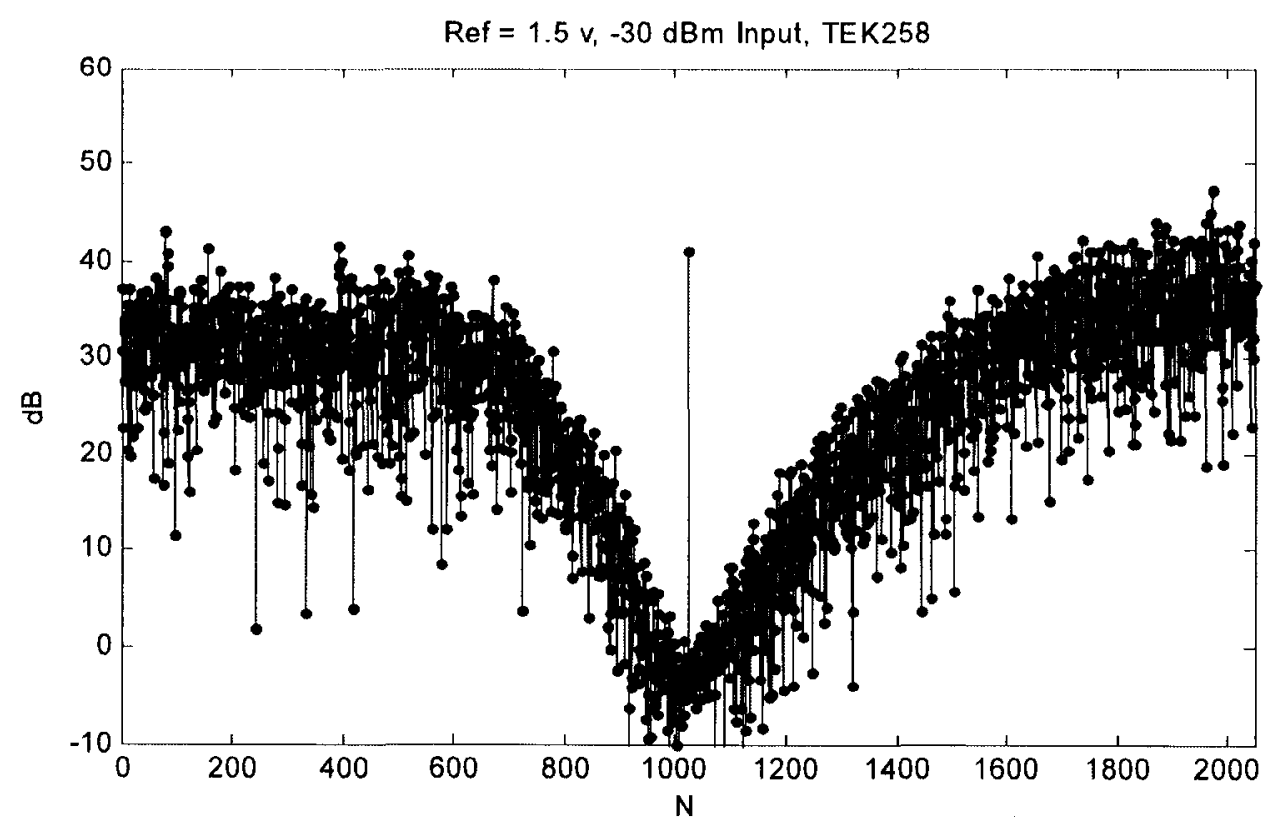

Fig. 7.6 Output Spectrum of a $-30 \mathrm{dBm}$ Input

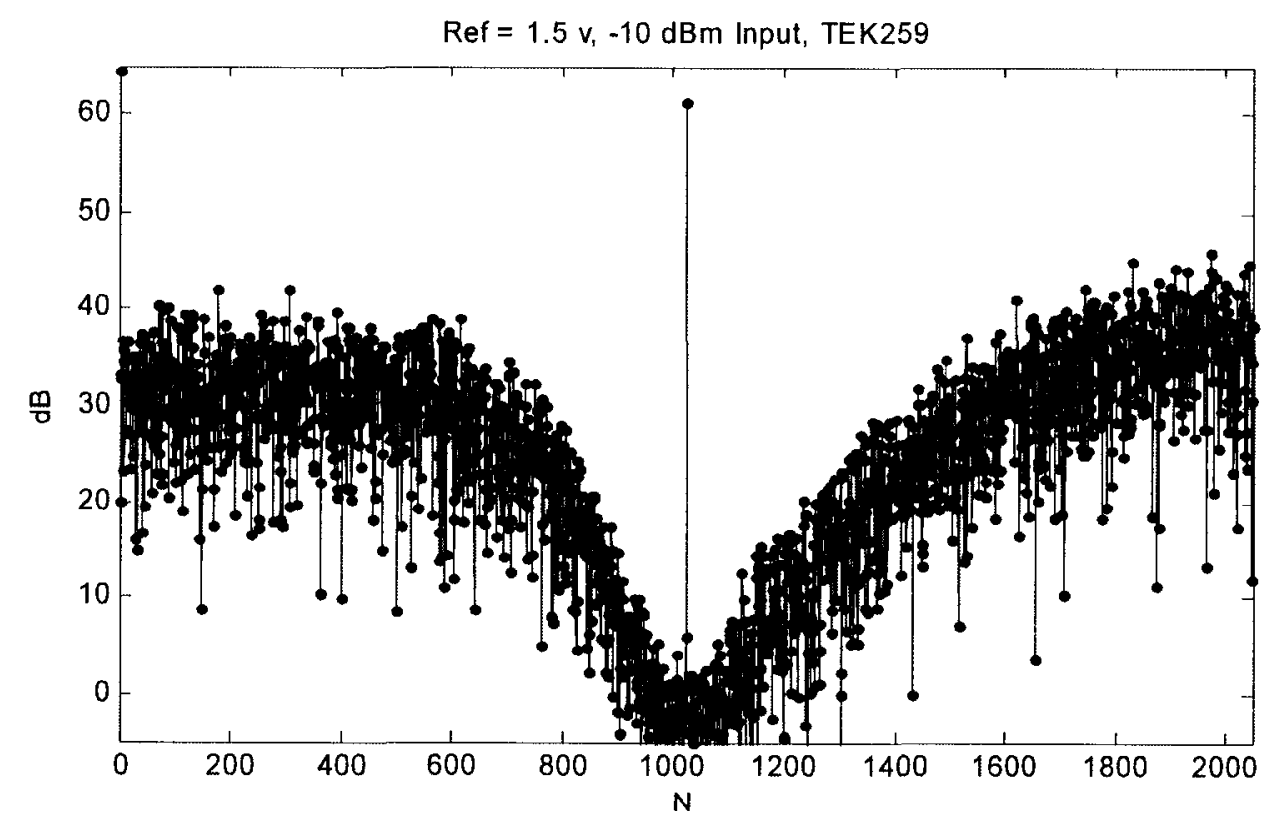

Fig. 7.7 Output Spectrum of a $-10 \mathrm{dBm}$ Input

In the above three figures, the $\mathrm{X}$-axis shows 0 to 2048 points, corresponding to 0 to 6.25 $\mathrm{MHz}$, which is the first Nyquist zone for a sampling rate of $12.5 \mathrm{MHz}$. The analog input 
is a $3.125 \mathrm{MHz}$ tone signal, corresponding to 1024 on the $\mathrm{X}$-axis. In Figures 7.5 through 7.7, the input level is $-60 \mathrm{dBm},-30 \mathrm{dBm}$, and $-10 \mathrm{dBm}$, respectively. The reference voltage was set at 1.5 volt. Since the output signal is digital, it is plotted in $\mathrm{dB}$ as is usually practiced.

Tests for different input levels from $-65 \mathrm{dBm}$ to $10 \mathrm{dBm}$ were conducted and the SNR over a $500 \mathrm{kHz}$ bandwidth was calculated. Figure 7.8 presents the SNR plot.

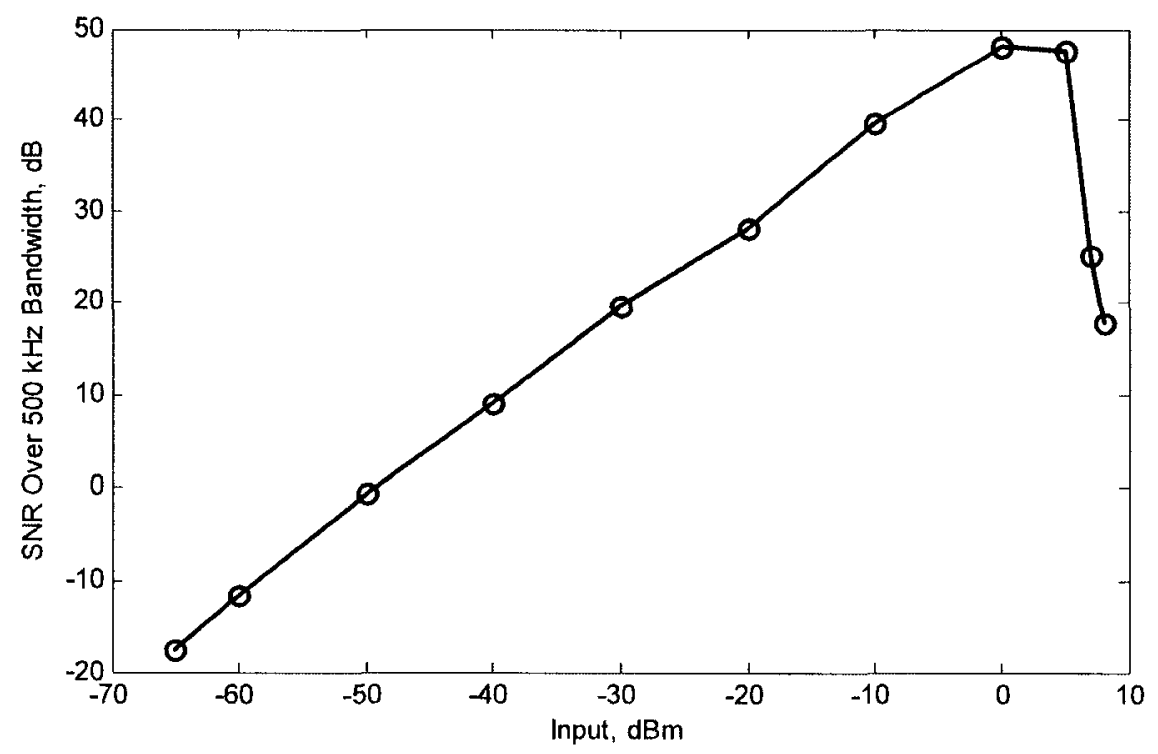

Fig. 7.8 SNR over $500 \mathrm{kHz}$ Bandwidth for the SDM Prototype

The Maximum SNR obtained was $48 \mathrm{~dB}$. For comparison, the ideal SNR was simulated for the discrete \& ideal version of the SDM without any thermal noise added. The ideal SNR is about $57.4 \mathrm{~dB}$. The $9.4 \mathrm{~dB}$ difference between the measured and the ideal SNR can be considered as being due to noise and non-ideality contributions from the discrete circuits, including D/A element mismatches. Overall the novel biquad circuit and cascaded loop filter based upon the biquad were proven to be feasible for continuoustime sigma-delta modulators. 


\subsubsection{Test of a Varying Reference Voltage}

Experimental tests were conducted to highlight two advantageous scenarios which benefit from the new reference feedback SDM. First performance over a range of temperatures that causes shifts in the reference level is demonstrated. Second the intentional change of the reference level for dynamic range control is presented. Conventional SDMs have difficulty doing this because it requires re-matching of the signal level around the SDM loop. On the other hand, conventional Nyquist-rate ADCs do allow, in principle, the change of reference level for such a purpose. However, in practice, the normal range of voltage swing usually fully occupies many reference levels in the ADC, not leaving much room for such a purpose.

Figures 7.9 through 7.11 present three examples of the test results. It is observed that as the reference varies, the signal scale changes accordingly, but the noise-shaping stays the same for the reference feedback SDM. As will be demonstrated in the next section, this is not the case for the conventional SDM.

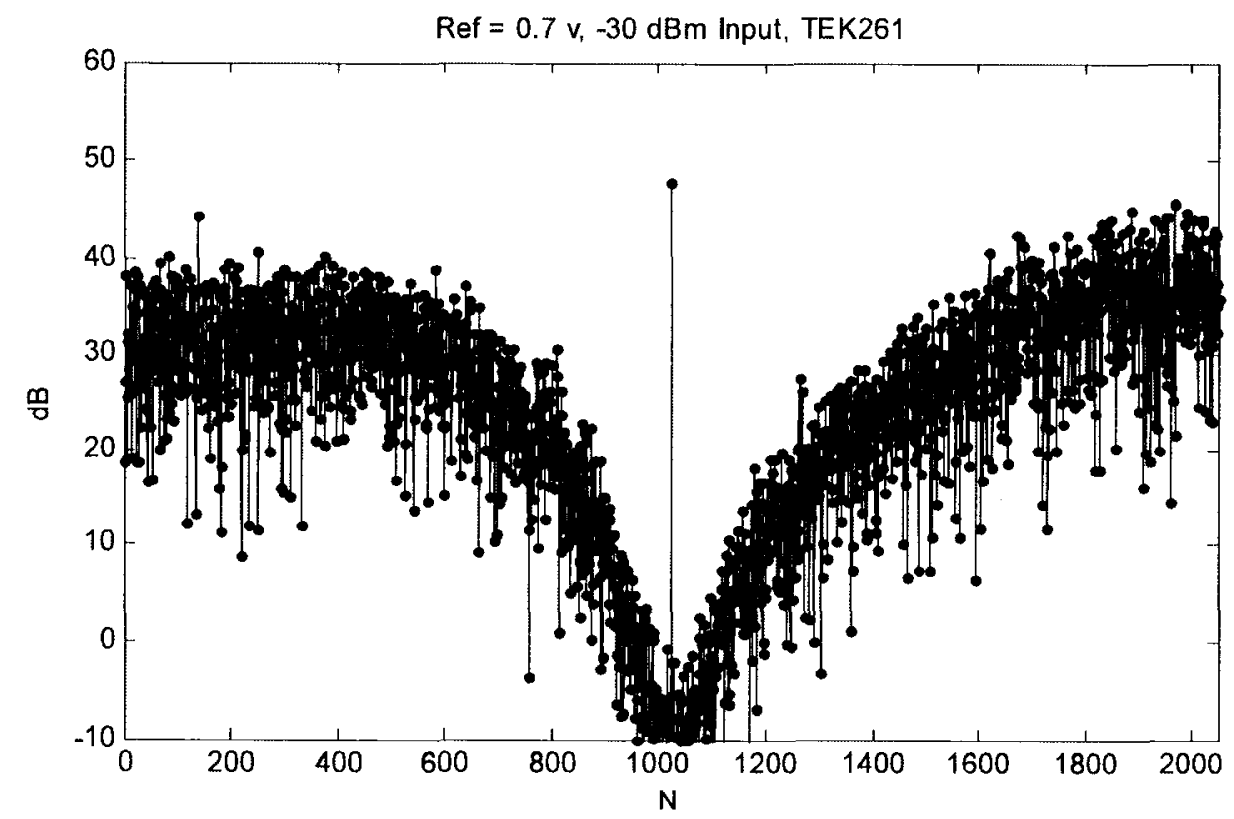

Fig. 7.9 Noise-Shaping Spectrum with the Reference Being 0.7 volt 


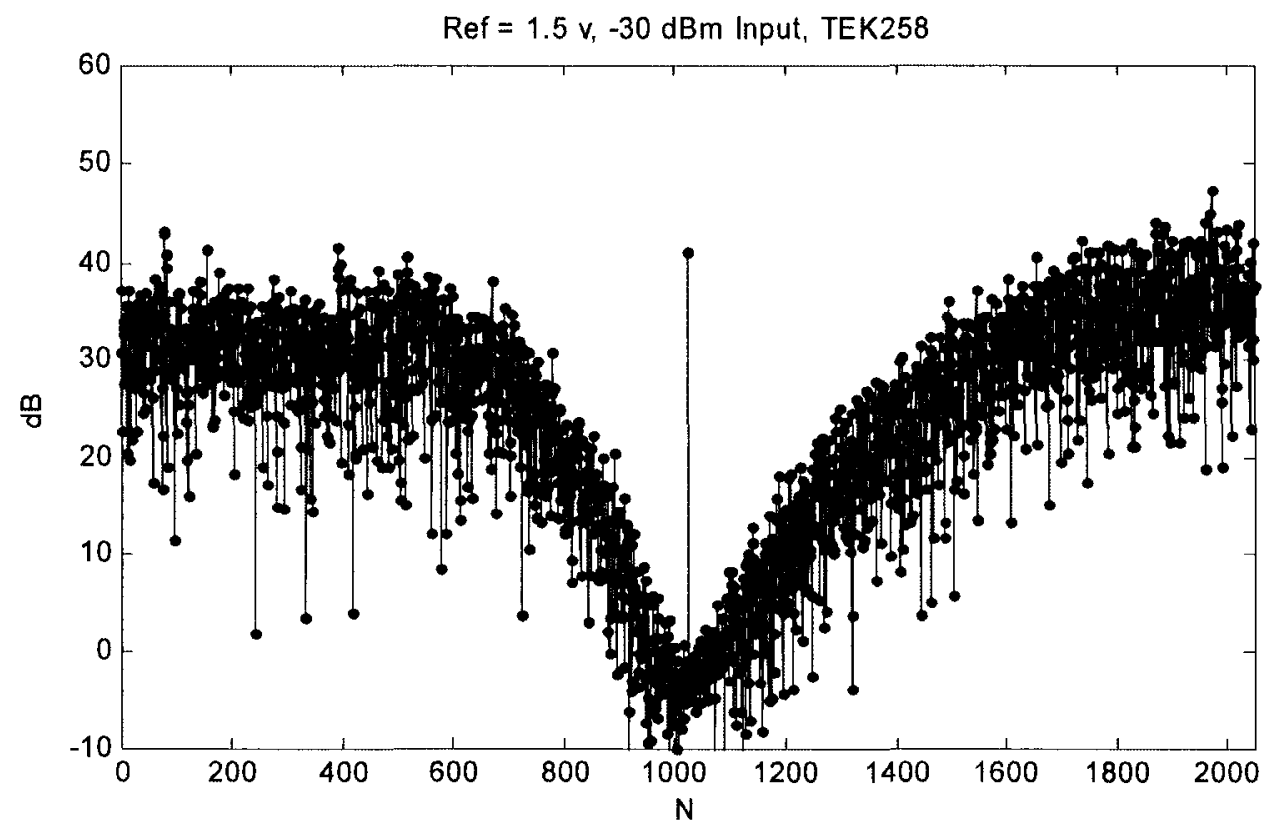

Fig. 7.10 Noise-Shaping Spectrum with the Reference Being 1.5 volt

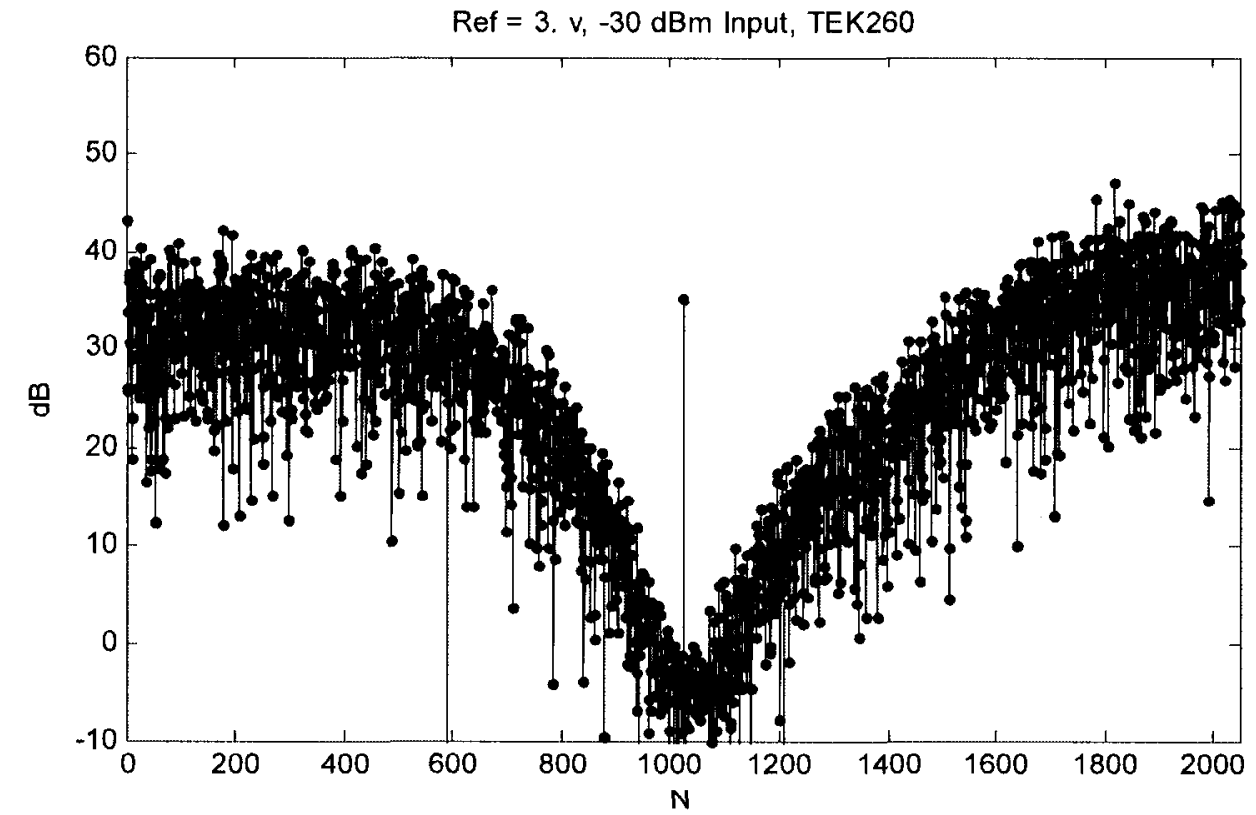

Fig. 7.11 Noise-Shaping Spectrum with the Reference Being 3 volt 
In Figures 7.9 through 7.11, the reference was set at 0.7, 1.5, and 3 volt, respectively, for an input level of $-30 \mathrm{dBm}$. The $\mathrm{X}$-axis shows 0 to 2048 points, corresponding to 0 to $6.25 \mathrm{MHz}$. The analog input is again a $3.125 \mathrm{MHz}$ tone signal, corresponding to $1024 \mathrm{on}$ the $\mathrm{X}$-axis. Since the SDM output signal is digital, it is plotted in $\mathrm{dB}$ as is the_usual practice. Almost the same noise-shaping spectrum can be seen in the three figures, validating the insensitivity of the reference feedback SDM to a change of reference level.

\subsubsection{Noise-Shaping Comparison with Conventional Feedback}

Similar tests of varying the reference voltage were also conducted for the SDM with a conventional summing-type DAC connected in the feedback path. Figure 7.12 shows the typical noise-shaping spectrum of the SDM with a reference of 1 volt.

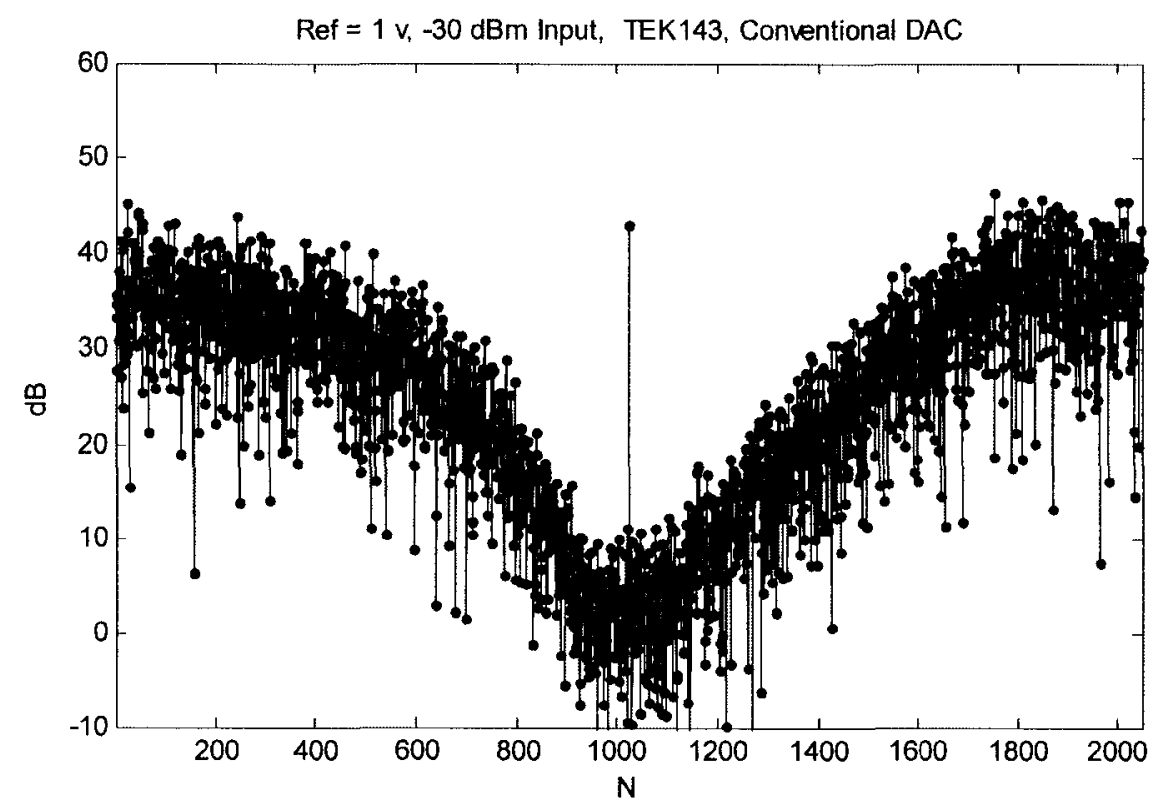

Fig. 7.12 Output Spectrum of the SDM with a Conventional DAC, Ref. $=1$ volt

In Figure 7.12, the reference was set at 1 volt for an input level of $-30 \mathrm{dBm}$. The $\mathrm{X}$-axis shows 0 to 2048 points, corresponding to 0 to $6.25 \mathrm{MHz}$. The analog input is a_3.125 MHz tone signal, corresponding to 1024 on the $\mathrm{X}$-axis. Since the output signal is digital, it is plotted in $\mathrm{dB}$. 


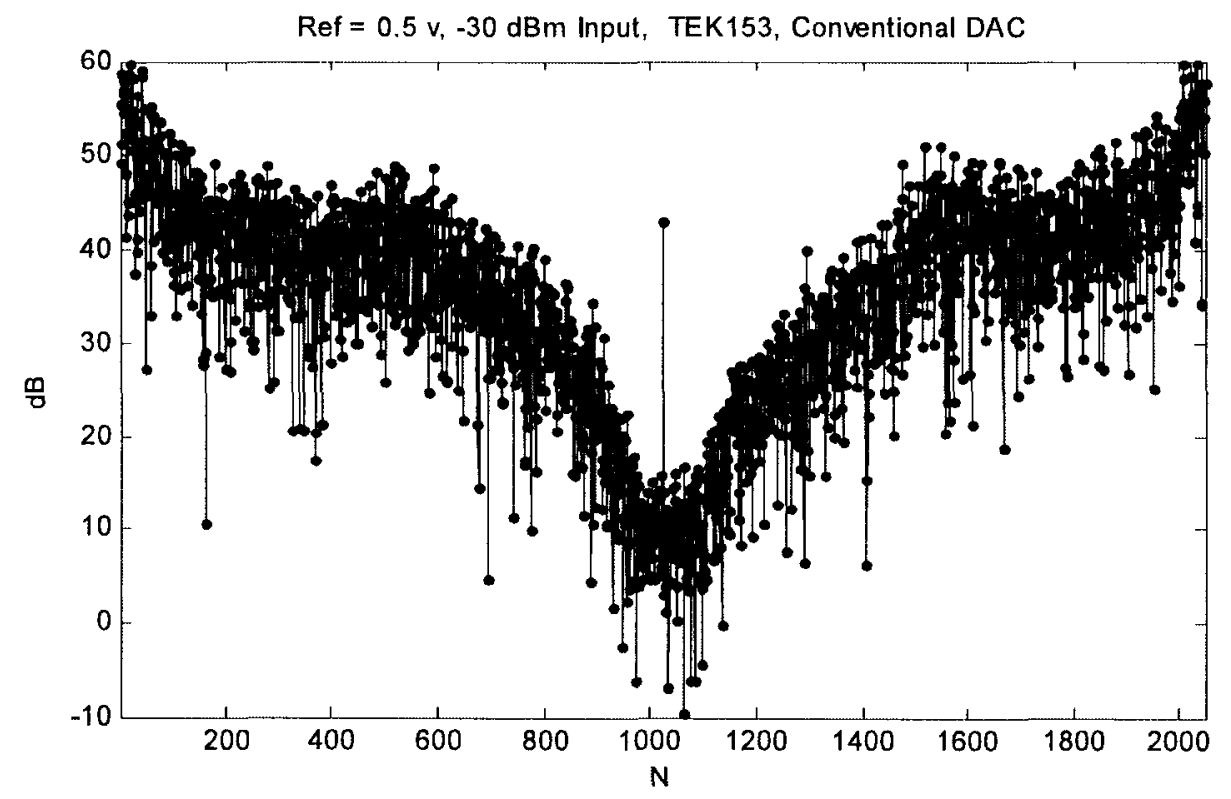

Fig. 7.13 Output Spectrum of the SDM with a Conventional DAC, Ref. $=0.5$ volt

Figure 7.13 shows the spectrum when the reference is reduced from 1 volt to 0.5 volt. A significant increase in noise can be observed. About $14 \mathrm{~dB}$ degradation in SNR results because of the reference change.

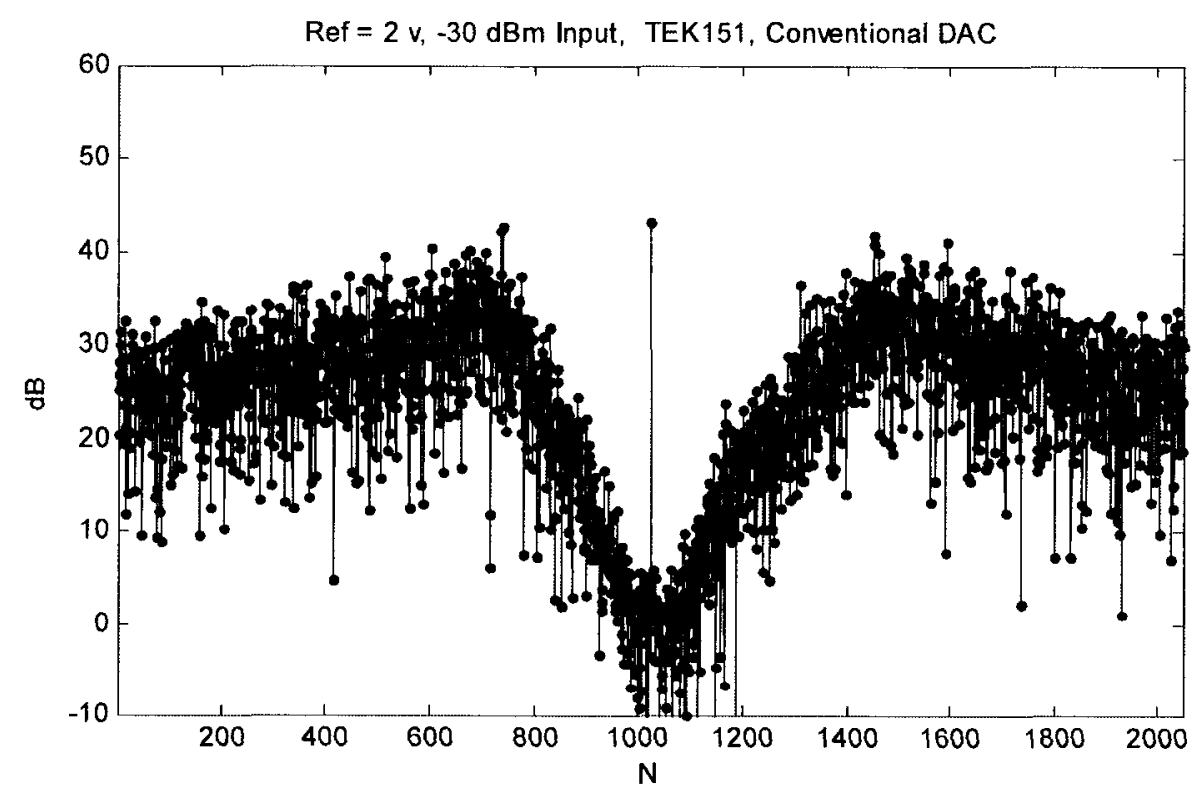

Fig. 7.14 Output Spectrum of the SDM with a Conventional DAC, Ref. $=2$ volt 
Figure 7.14 shows the spectrum when the reference increases from 1 volt to 2 volt. Again a degraded noise-shaping can be observed. Compared to Figure 7.12, the passband is noticeably narrower. Within the passband, no considerable change in SNR is observed. This is in contrast to the reduced reference case where the SNR is degraded drastically. This agrees with the analysis performed in Section 5.6 (Figure 5.14).

\subsubsection{SNR Comparison with Conventional Feedback}

Figures 7.15 through 7.17 are examples of the output spectrum of the SDM but with a conventional summing-type DAC connected in the feedback path.

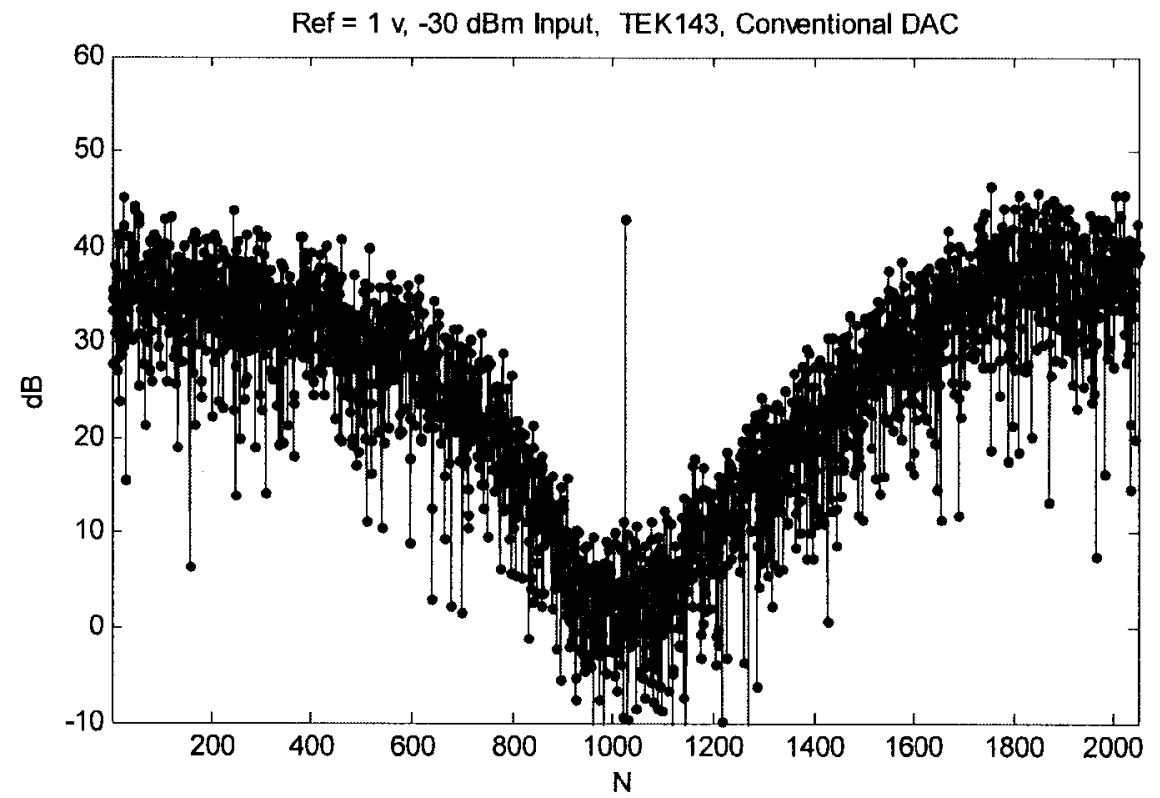

Fig. 7.15 Output Spectrum of the SDM with a Conventional DAC, $-30 \mathrm{dBm}$ Input

In Figures 7.15 through 7.17, the reference was set at 1 volt for an input level of -30 $\mathrm{dBm},-50 \mathrm{dBm}$, and $-10 \mathrm{dBm}$, respectively. The $\mathrm{X}$-axis shows 0 to 2048 points, corresponding to 0 to $6.25 \mathrm{MHz}$. The analog input is again $3.125 \mathrm{MHz}$ tone signal, corresponding to 1024 on the $\mathrm{X}$-axis. Since the output signal is digital, it is plotted in $\mathrm{dB}$. 


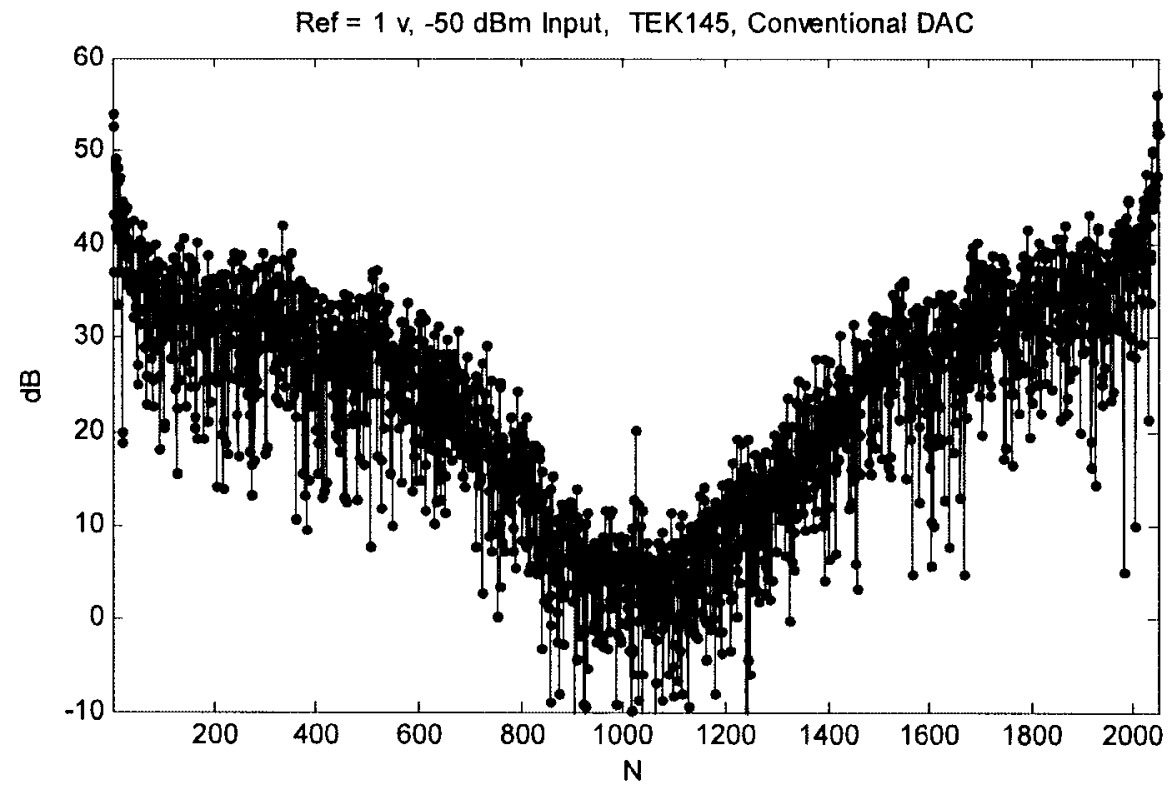

Fig. 7.16 Output Spectrum of the SDM with a Conventional DAC, $-50 \mathrm{dBm}$ Input

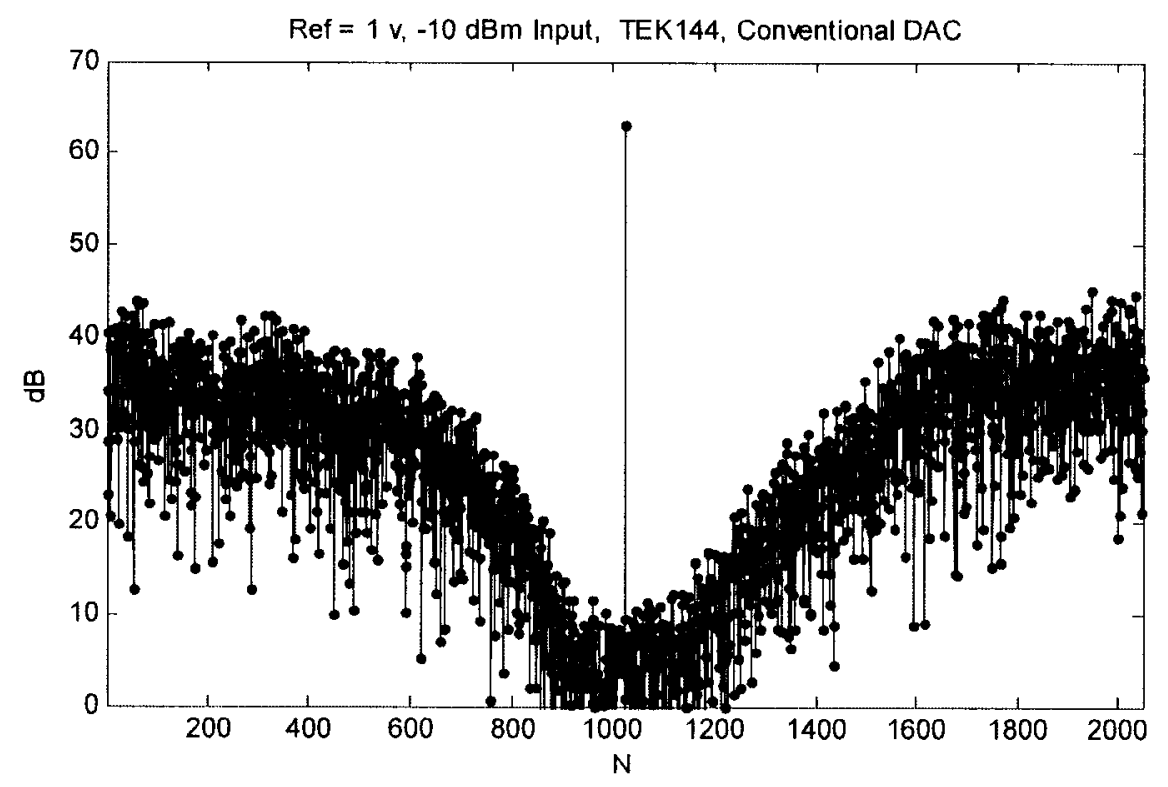

Fig. 7.17 Output Spectrum of the SDM with a Conventional DAC, $-10 \mathrm{dBm}$ Input 
Again a SNR test was conducted with input from $-57 \mathrm{dBm}$ to $9 \mathrm{dBm}$ for the conventional DAC connected in the SDM. The SNR plot is shown in Figure 7.18 along with the one for the reference feedback SDM. The SNR of the reference feedback SDM is 5 to $10 \mathrm{~dB}$ better than that of the conventional DAC SDM. An explanation for the relatively poor performance of the conventional DAC SDM is that the operational amplifier in the conventional DAC contributes significant thermal noise compared to that of the CMOS switches in the reference feedback SDM.

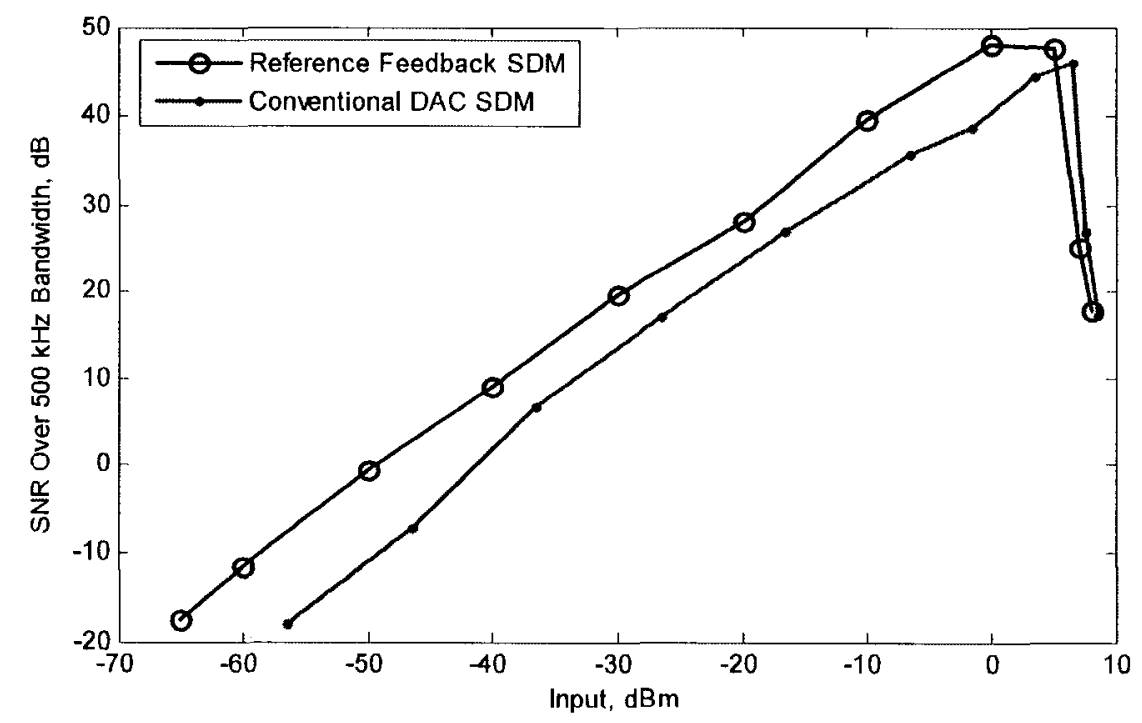

Fig. 7.18 SNR Plot of the Conventional DAC SDM versus the New SDM

\subsubsection{Two-Tone Test of the Reference Feedback SDM}

Two-tone tests were also conducted for the reference feedback SDM prototype. The test results are shown in Figures 7.19 through 7.23. The noise spectrum of the two-tone tests is about the same shape, with the same level of spikes as that of single-tone test (Figure 7.6). The concept of third-order products is not applicable to SDM, since its nonlinearity is not necessarily of a polynomial type. 


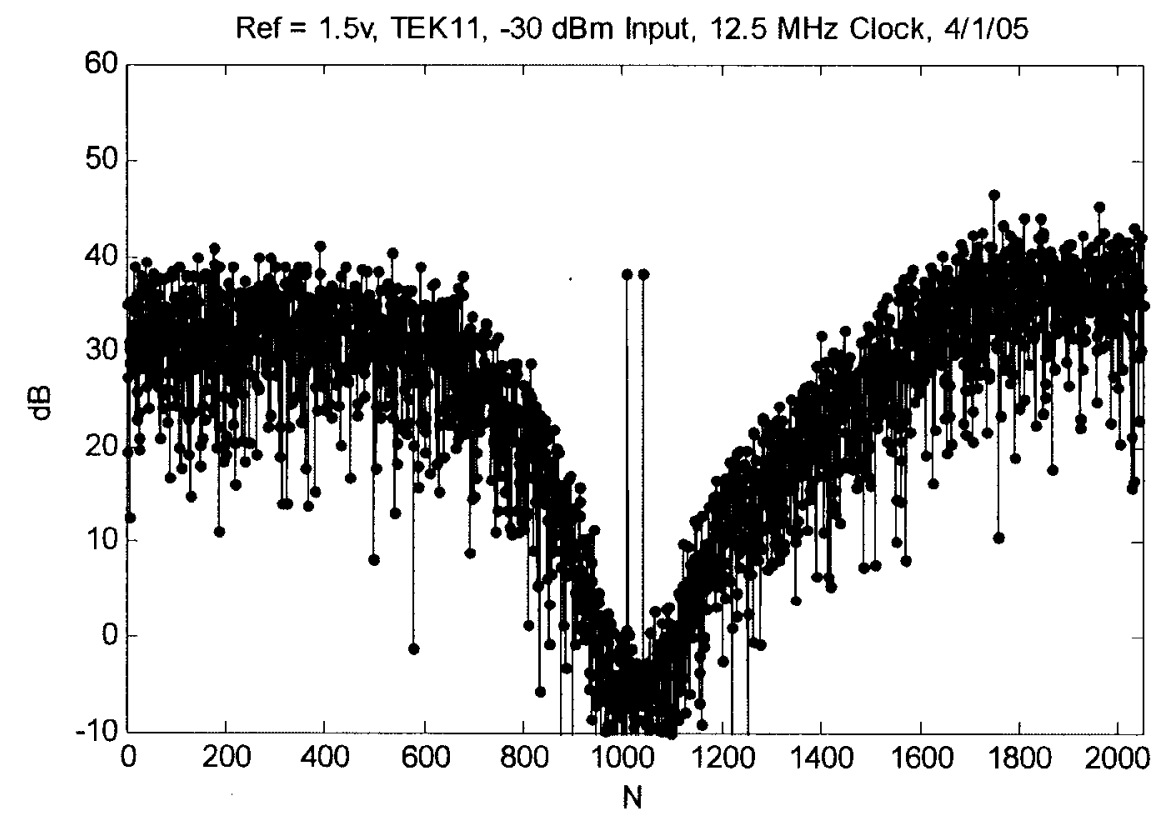

Fig. 7.19 Two-Tone Test with a Tone Spacing of $97656 \mathrm{~Hz}$

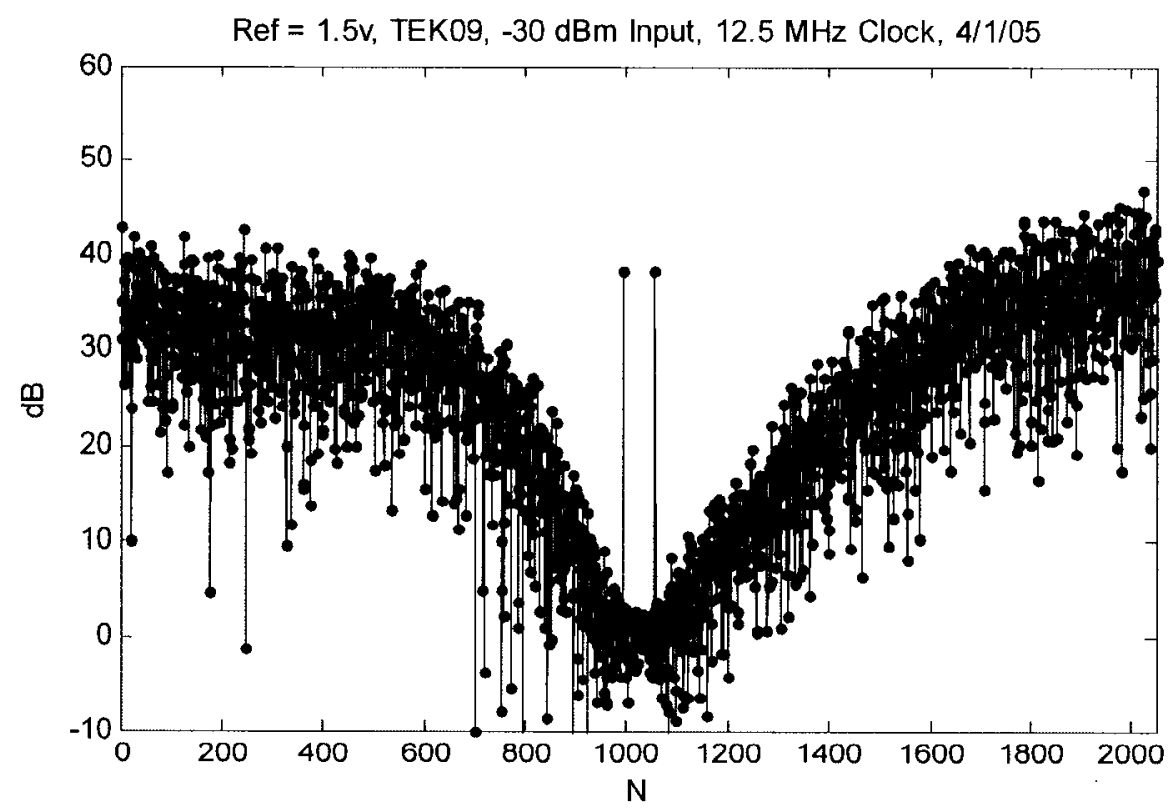

Fig. 7.20 Two-Tone Test with a Tone Spacing of $195312 \mathrm{~Hz}$ 
In Figures 7.19 and 7.20 , the reference was set at 1.5 volt for two inputs of $-30 \mathrm{dBm}$ spaced by $97656 \mathrm{~Hz}$ and $195312 \mathrm{~Hz}$, respectively. The X-axis shows 0 to 2048 points, corresponding to 0 to $6.25 \mathrm{MHz}$. Since the output signal is digital, it is plotted in $\mathrm{dB}$.

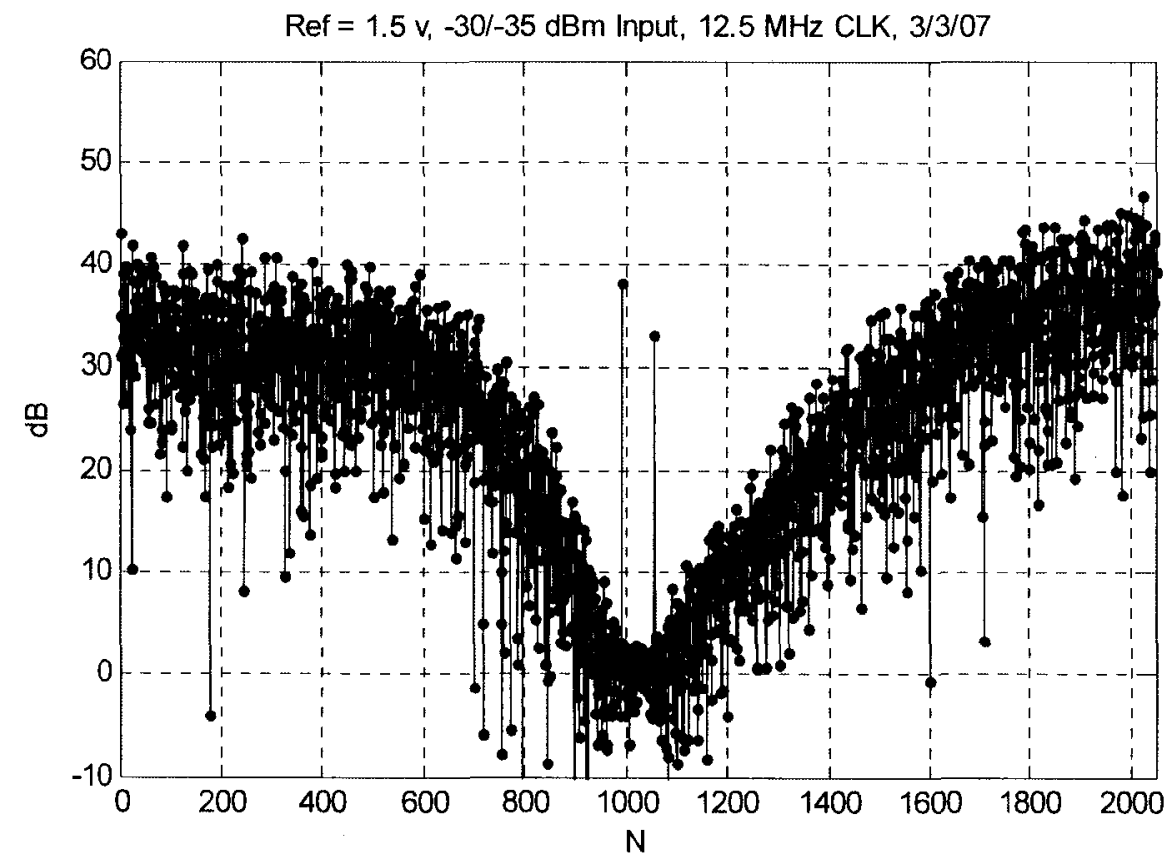

Fig. 7.21 Two-Tone Test with $5 \mathrm{~dB}$ Difference and Tone Spacing by $195312 \mathrm{~Hz}$

In Figure 7.21, the reference was set at 1.5 volt for two inputs of $-30 \mathrm{dBm}$ and $-35 \mathrm{dBm}$, respectively. The tone spacing is $195,312 \mathrm{~Hz}$. The X-axis shows 0 to 2048 points, corresponding to 0 to $6.25 \mathrm{MHz}$. Since the output signal is digital, it is plotted in $\mathrm{dB}$. At the output, the two tones were measured with a difference of $5.08 \mathrm{~dB}$, when the two tones at the input differed by $5 \mathrm{~dB}$.

In Figure 7.22, the two tone inputs were changed to $-30 \mathrm{dBm}$ and $-40 \mathrm{dBm}$, respectively. And at output, the two tones were measured with a result of $9.96 \mathrm{~dB}$ difference, (for a difference in the two tone inputs of $10 \mathrm{~dB}$ ).

In Figure 7.23, the two tone inputs were changed to $-30 \mathrm{dBm}$ and $-45 \mathrm{dBm}$, respectively. And at output, the two tones were measured with a result of $15.1 \mathrm{~dB}$ difference, (for a difference in the two tone inputs of $15 \mathrm{~dB}$ ).

These measurements suggest that the 3-bit reference feedback SDM has very good linearity. 


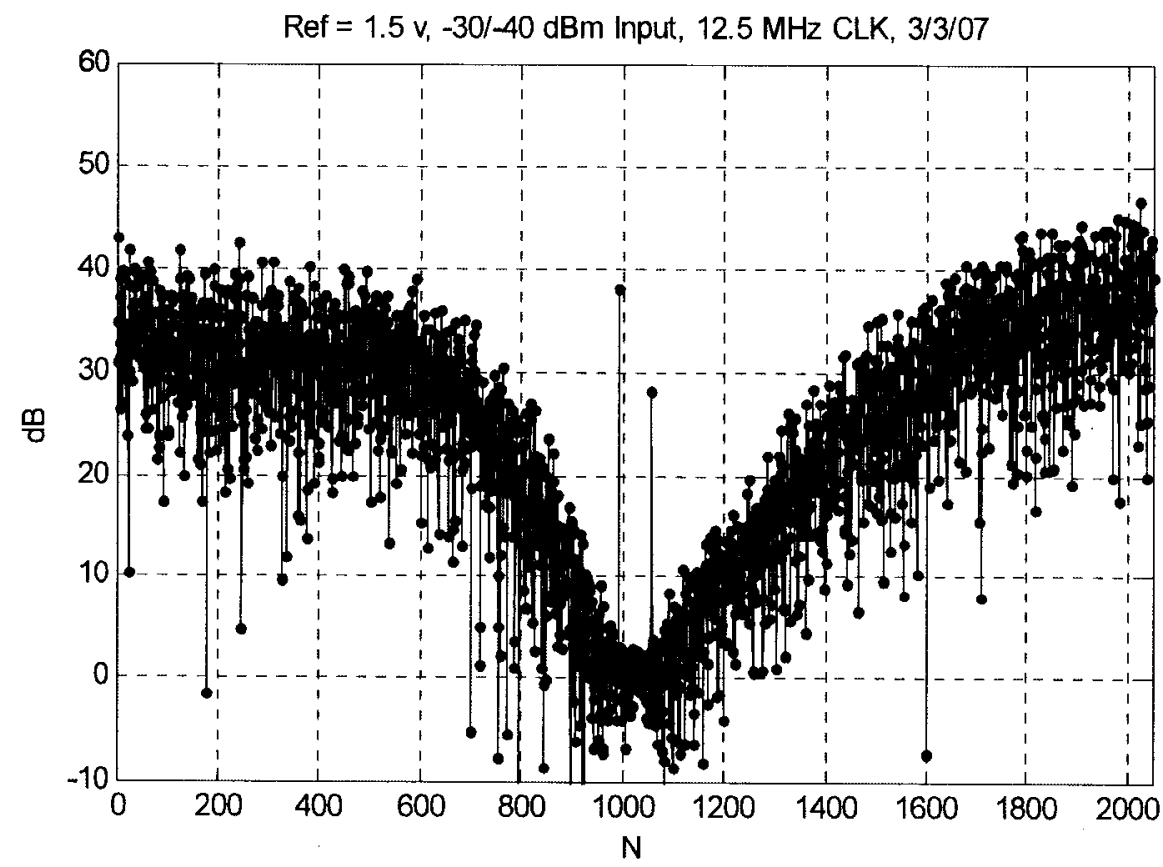

Fig. 7.22 Two-Tone Test with $10 \mathrm{~dB}$ Difference and Tone Spacing by $195312 \mathrm{~Hz}$

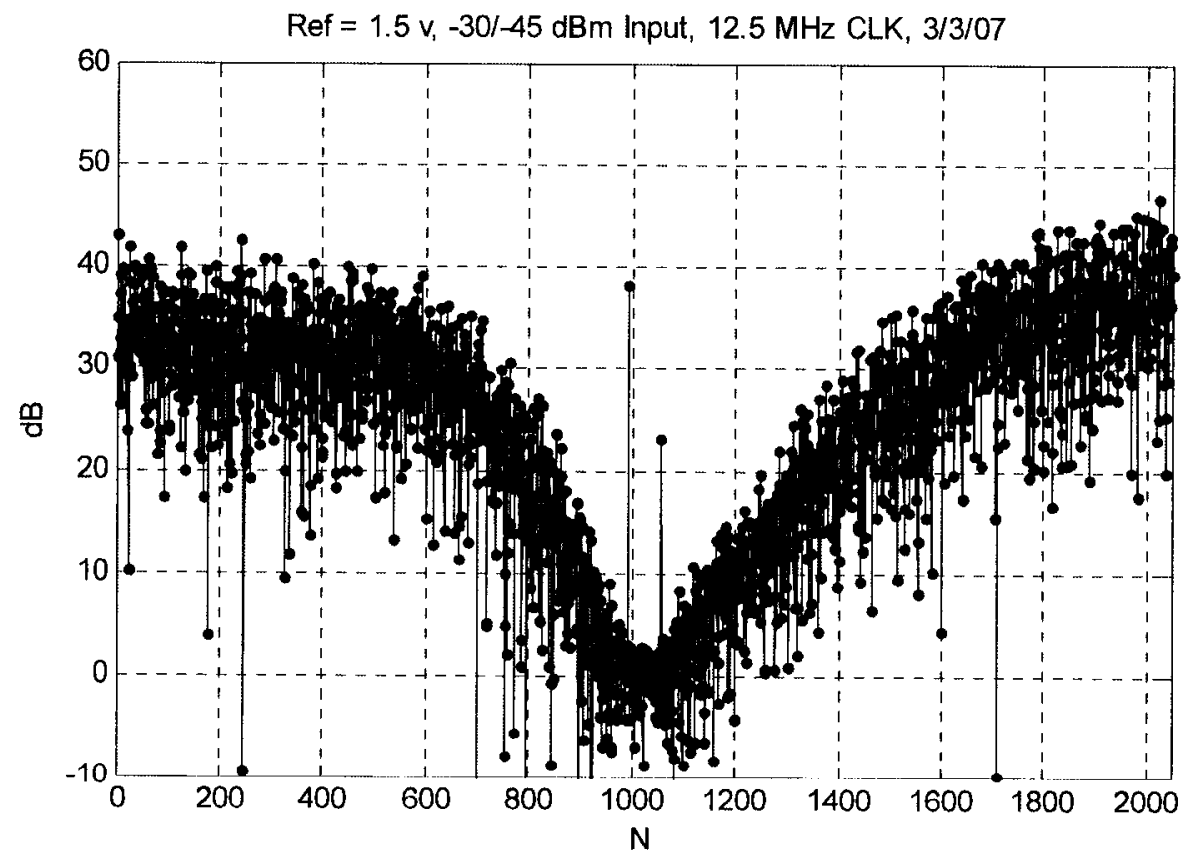

Fig. 7.23 Two-Tone Test with $15 \mathrm{~dB}$ Difference and Tone Spacing by $195312 \mathrm{~Hz}$ 


\subsection{Summary}

The fourth order 3-bit CT bandpass reference feedback SDM was built and experimentally evaluated along with a conventional feedback SDM having a summingtype D/A in the feedback path. The Maximum SNR of $48 \mathrm{~dB}$ was obtained within a 500 $\mathrm{kHz}$ bandwidth. The 3-bit reference feedback SDM shows a very stable noise-shaping performance over a wide range of reference level (up or down by 100\%); in contrast, significant distortion and dynamic range and bandwidth loss were observed for the conventional feedback SDM. The SNR of the reference feedback SDM is around $7 \mathrm{~dB}$ better than that of the conventional feedback SDM, suggesting that the summing-type $\mathrm{D} / \mathrm{A}$ in the conventional feedback SDM contributes more noise than the reference feedback circuit. Finally, two-tone tests showed good linearity for the 3-bit reference feedback SDM. 


\section{CHAPTER 8 Conclusions and Future Work}

\subsection{Conclusions}

Interference and crowded channels make radio design a very challenging task, requiring precise frequency conversion and large amounts of filtering. SDM oversampling offers an efficient solution for $A / D$ conversion in digital radios, because only the desired channel is captured through noise-shaping.

Sampling rate conversion in a digital signal processing is equivalent to frequency conversion in an analog circuit. In the digital radios, the output data of $A / D$ converters are often subject to sampling-rate-conversion. The new clock structural model provided in Chapter 4 can be a helpful tool in designing efficient sampling-rate-conversion digital filters.

The Quantizer in a SDM cannot be modeled as a linear device by itself, because only a few bits are employed. To understand the mechanism of sigma-delta modulation, stability and linearity of the SDM loop must be considered together. In a properly designed SDM loop, stability and linearity reinforce each other through feeding back a noise-like output signal to the quantizer, forcing it to operate in a linear manner.

A multibit SDM has a wider bandwidth than its 1-bit counterpart, because a full gain noise-shaping loop can be implemented in the multibit SDM. In comparison, for second-order above 1-bit SDMs, noise-shaping gain has to be reduced to preserve loop stability. A quantitative comparison of the multibit vs the 1-bit SDMs, conducted in some real-world scenario simulations, shows that at moderate levels of element mismatch, a multibit SDM is better than a 1-bit SDM for bandwidths above $500 \mathrm{kHz}$. This suggests that the multibit SDM is a better choice for broadband applications. 
Signal mismatch in a SDM loop causes distorted noise-shaping, as well as dynamic range and/or bandwidth loss. The reference feedback SDM offers a signalmatched noise-shaping loop over process and temperature variations, which is advantageous for reference circuit design and dynamic range \& AGC control. AGC control using an intentional change of reference level cannot be achieved in a conventional feedback SDM, and is not practical in a Nyquist-rate A/D converter because its limited voltage range is already occupied by a lot of quantization levels.

A fourth order 3-bit CT bandpass reference feedback SDM was designed and experimentally evaluated. The CT loop filter of the 3-bit reference feedback SDM was implemented by cascading biquad sections, for which a novel biquad circuit was developed. This novel biquad circuit allows any higher order CT loop filter to be reduced to second-order sections, and each section can be designed and tuned independently.

As a side note, the successful implementation of the 3-bit reference feedback multibit SDM using discrete components also suggests a low cost way of building a high performance ADC with off-the-shelf components for low volume applications.

\subsection{Future Work}

A discrete, simple resistor string voltage divider was used in the experimental prototype as a reference circuit. The reference circuit can also be realized as a band-gap circuit, which is a preferred implementation at the IC level. Since the band-gap circuit has much better matching property than capacitors and resistors, further reduced element mismatch can potentially be realized. This IC level implementation would be a good choice for future works.

The novel biquad circuit makes the design and realiztion of higher order loop filters much easier. An implementation of the novel biquad circuit at the IC level would be a meaningful undertaking. 


\section{Reference}

[1] J. C. Candy and G. C. Temes, "Oversampling sigma-delta data converters", IEEE Press, New York 1992.

[2] R. Carely, "A noise-shaping coder topology for 15+ bit converter," IEEE Journal of Solid-State Circuits, pp. 267-273, April, 1989.

[3] B. Boser and B. Wooley. "The design of sigma-delta modulation analog-to-digital converters". IEEE J. of solid-state circuits, vol. 23, NO. 6, Dec, 1988.

[4] J. Mitola. "The software radio". IEEE Nat'l. Telesys. Conf. 1992.

[5] O. Shoaei, "Continuous-time sigma-delta A/D converters for high speed applications," Ph.D. dissertation, Carleton Univ., Ottawa, Canada, 1996.

[6] J. A. Cherry and W. M. Snelgrove, "Excess loop delay in continuous-time sigma-delta modulators," IEEE trans. on Circuits and Systems, vol. 46, No. 4, April, 1999.

[7] J. G. Proakis and D. G. Manolakis, Digital Signal Processing: Principles, Algorithms, and Applications. Prentice-Hall: New Jersey, 1996.

[8] R. G. Vaughan, N. L. Scott, and D. R. White, "The Theory of Bandpass Sampling", IEEE Trans. On Signal Processing, vol. 39, No. 9, pp. 1973-1984, Sept., 1991.

[9] T. S. Rappaport, Wireless Communications: principles and practices. Prentice Hall: New Jersey, 1996.

[10] S. Haykin, Communication Systems. John Wiley \& Sons, New York, 2000.

[11] J.G.Proakis, Digital Communications. McGraw-Hill, 1995.

[12] D. Shen, et al., "A 900-MHz RF front-end with integrated discrete-time filtering", IEEE J. of Solid-State Circuits, vol. 31, no. 12, pp. 1945-1954, Dec. 1996.

[13] W. L. Lee, “A novel higher order interpolative modulator topology for high resolution oversampling A/D converter,"' Master's thesis, Massachusetts Inst. Of Technology, Cambridge, June 1987.

[14] J. Min, et al., "An all CMOS architecture for a low-power frequency-hopped 900 MHz spread spectrum transceiver," Proc. IEEE Custom Integrated Circuits Conf., pp. 379-382, May 1994 
[15] P. Y. Chan, et al., "A highly linear 1-GHz CMOS downconversion mixer", European Solid-State Circuits Conf., Sevilla, Spain, pp. 210-213, 1993.

[16] A. A. Abidi, "Low-power radio-frequency IC's for portable communications”, Proc. Of IEEE, Vol. 83, no. 4, pp 544-569, Apr. 1995.

[17] A. A. Abidi, "Direct-conversion radio transceivers for digital communications", IEEE J. of Solid-State Circuits, vol. 30, no. 12, pp. 1399-1410, Dec. 1995.

[18] R. Coy, et al., "HF-based radio receiver design based on digital signal processing", Electronics and communication Engineering Journal, pp. 82-90, Apr 1992.

[19] J. Cherry, "Theory, practice, and foundamental performance limits of high-speed data conversion using continuous-time sigma-delta modulators", Ph.D. Dissertation, Carleton Univ., 1999.

[20] B. Friend et al., "Methods and Apparatus for Distributing Mismatched Error Associated with Data Converter Elements", U.S. Pat. No. 6218977, issued Apr. 17, 2001 .

[21] R. T. Baird and T. S. Fiez, "Linearity enhancement of multibit sigma-delta A/D and D/A converters using data weighted averaging," IEEE Transactions of Circuits and Systems II, pp. 753-762, December, 1995.

[22] K. Chen et al., "Multi-bit Sigma-delta Converters Employing Dynamic Element Matching With Reduced Baseband Tones", U.S. Pat. No. 6304608, Oct. 16, 2001.

[23] H. R. Karimi and B. Friedrichs, "Wideband digital receivers for multi-standard software radios", IEE Colloquium on adaptable and multi-standard mobile radio terminals, Oct. 1997.

[24] H. R. Karimi, N. W. Anderson, and P. McAndrew, "Digital signal processing aspects of software definable radios", IEE Colloquium, London, March 1998.

[25] S. Kourtis, P. McAndrew, and P. Tottle, "Software radio 2G \& 3G inner receiver processing", IEE Colloquium, London, 1999.

[26] W. H. W. Tuttlebec, "Software radio technology: A European perspective", IEEE Communications Magazine, pp. 118-123, Feb. 1999.

[27] A. E. Suominen, "Simplified high frequency tuner and tuning method", U.S. Pat. No. 5937341, Filed Sept. 13, 1996, Issued Aug. 10, 1999. 
[28] G. F. Schroeder and G. Ellis, "Digital demodulator for frequency-divisionmultiplexed signals", U.S. Pat. No. 4884265, Filed April 30, 1987, Nov. 28, 1989.

[29] R. W. Caulfield, F. Harris and W. McKnight, "Digital mixing to baseband decimation filter", U.S. Pat. No. 5757867, Filed March. 30, 1995, May 26, 1998.

[30] H. S. Jackson, "Bandpass sigma-delta analog-to-digital converter (ADC), method therefore, and receiver using same", U.S. Pat. No. 5442353, Filed Oct. 25, 1993, Issued Aug. 15, 1995.

[31] W. S. Song, "Efficient polyphase quadrature digital tuner", U.S. Pat. No. 6049573 , Filed Dec. 17, 1997, Issued April 11, 2000.

[32] P. H. Gailus, W. J. Turney and F. R. Yester, "Method and arrangement for sigmadelta converter for bandpass signals", U.S. Pat. No. 4857928, Filed Jan. 28, 1988, Issued Aug. 15, 1989.

[33] T. A. Williams, "Bandpass sigma-delta converter suitable for multiple protocols", U.S. Pat. No. 5345406, Filed Aug. 25, 1992, Issued Sept. 6, 1994.

[34] R. Schreier and W. M. Snelgrove, "Decimation for bandpass sigma-delta analog-todigital conversion", Proc. IEEE, Int. Symp. On Circuit and Systems, vol. 3, pp. 1801-1804, May 1990.

[35] P. P. Vaidyanathan, "Multirate digital filters, filter banks, polyphase networks, and applications: A tutorial", Proceedings of the IEEE, Vol 78, No.1, 1990.

[36] T. Hentschel and G. Fettweis, "Sample rate conversion for software radio", IEEE communication magazine, pp. 142-150, August, 2000.

[37] S. W. Smith, The scientist and engineer's guide to digital signal processing, California Technical Publishing, 1999

[38] S. Haykin and B. V. Veen, Signal and Systems, John Wiley \& Sons: New York, 1999.

[39] E. B. Hogenauer, "An economical class of digital filter for decimation and interpolation", IEEE trans of Acoustics, Speech, and Signal Processing, Vol. ASSP-29, April, 1981.

[40] R. E. Crochiere and L. R. Rabiner, "Interpolation and decimation of digital signals a tutorial review", Proc. IEEE, vol. 69, No. 3, March 1981. 
[41] S. R. Norsworthy, R. Schreier and G. C. Temes, Sigma-delta Data Converter, (chapter 9), IEEE Press, 1996.

[42] S. A. Jantzi, W. M. Snelgrove and P. F. Ferguson, "A Fourth-order Bandpass Sigmadelta Modulator", IEEE J. solid-state circuits, vol. 28, no. 3, March 1993.

[43] J.C.Rudell et. al., "A 1.9-GHz wideband IF double conversion CMOS integrated receiver for cordless telephone applications" IEEE J. Solid-State Circuits, vol. 32, no. 12, pp. 2071-2088, 1997.

[44] P.R. Gray and R.G. Meyer, "Future Directions in Silicon ICs for RF Personal Communications", Custom Integrated Circuits Conference, pp. 83-90, May, 1995

[45] J. C. Rudell, et al., "Recent developments in high integration multi-standard CMOS transceiver for personal communication systems", International Symposium on Low Power Electronics, Monterey, California, 1998.

[46] R. Baines, "the DSP Bottleneck", IEEE communications magazine, pp. 46-54, May 1995.

[47] J. Crols and M. S. J. Steyaert, "Low-IF topologies for high-performance analog front ends of fully integrated receivers", IEEE trans. on circuits and systems, vol. 45, No. 3, pp. 269-282, March, 1998.

[48] D. B. Chester, “Digital IF Filter Technology for 3G Systems: an Introduction”, IEEE communications magazine, pp. 102-107, Feb. 1999.

[49] J. A. Wepman, "Analog-to-Digital Converters and Their Applications in Radio Receiver", IEEE communications magazine, pp. 39-45, May 1995.

[50] A. K. Salkintzis, H. Nie, and P. T. Mathiopoulos, "ADC and DSP Challenges in the Development of Software Radio Base Stations", IEEE Personal Communications, Aug. 1999.

[51] T. Shui, R. Schreier, F. Hudson, "Mismatch Shaping for a Current-mode Multibit Sigma Delta DAC," IEEE J. of Solid-state Circuit, Vol. 34, pp. 331-338, March 1999.

[52] I. Fujimori et al, “A 90 dB SNR, 2.5 MHz Output Rate ADC Using Cascaded Multibit Delta-Sigma Modulation at 8x Oversampling Ratio," IEEE J.of Solid-state Circuits, Vol. 35, pp. 1820-1828, December 2000.

[53] "GC4016 Multi-Standard Quad DDC Chip Data Sheet”, Graychip Inc. Aug. 2000. 
[54] A. Tabatabaei and B. A. Wooley, "A two-path bandpass sigma-delta modulator with extended noise shaping", IEEE J. solid-state circuits, vol. 35, no. 12, pp. 1799-1809, Dec. 2000.

[55] R. Atarius and G. Frank, "Method and apparatus for estimating a frequency offset", U.S. Pat. No. 6104767, Filed Nov. 17, 1997, Issued Aug. 15, 2000.

[56] J. Stein, Digital Signal Processing --- a computer science perspective. John Wiley \& Sons, 2000.

[57] K. Vleugels, S. Rabii and B. A. Wooley, “A 2.5-V sigma-delta modulator for broadband communications applications", IEEE J. solid-state circuits, vol. 36, no. 12, pp. 1887-1899, Dec. 2001

[58] H. Meyr, M. Moeneclaey and S. A. Fechtel, Digital Communication Receivers --synchronization, channel estimation, and signal processing, John Wiley \& Sons: New York, 1997.

[59] A. N. D’Andrea and U. Mengali, "A Simulation study of clock recovery in QPSK and 9 QPRS systems," IEEE Trans commun., vol. COM-33, pp. 1139-1142, Oct. 1985.

[60] A. N. D'Andrea and U. Mengali, "Performance analysis of the delay-line clock regenerator" IEEE Trans commun., vol. COM-34, pp. 321-328, Apr. 1986.

[61] F. M. Gardner, "A transformation for digital simulation of analog filters" IEEE Trans. Communication, vol. 44, pp. 676-680, July 1986.

[62] O. Shoaei and W. M. Snelgrove "Design and implementation of a tunable $40 \mathrm{MHz}-$ $70 \mathrm{MHz}$ Gm-C bandpass $\Delta \Sigma$ modulator" IEEE Trans. on Circuits and Systems II: Analog and Digital Signal Processing, Volume: 44 , Issue: 7 , July 1997.

[63] J. Engelen and R. Plassche "Bandpass Sigma Delta Modulators" Kluwer Academic Publishers, Boston, 1999.

[64] J. Engelen, R. Plassche, E. Stikoort, and A. Venes, "A sixth-order continuous-time bandpass sigma-delta modulator for digital radio IF," IEEE J. solid-state circuits, vol. 34, no. 12, p1753-1764, December 1999.

[65] Kendall L. Su “Analog Filters", Kluwer Academic Publishers, Boston, 1996

[66] W. R. Bennett "Spectra of quantized signals", Bell System Technical Journal, vol. 27, pp. 446-472, 1948. 
[67] B. Widrow "A study of rough amplitude quantization by means of Nyquist sampling theory", IRE Trans. On Circuit Theory, pp. 266-276, Dec. 1956.

[68] B. Widrow, I. Kollar, and M. Liu "Statistical theory of quantization", IEEE Trans. on Instrumentation and Measurement, Vol. 45, pp.353 - 361, April 1996.

[69] Medeira, Perez-Verdu \& Rodriguez-Vazquez, "A 13-bit 2.2-MS/s, 55-mW multibit cascade SDM in CMOS 0.7-um single-poly technology," J. Solid-State Circuits, p.748-760, June 1999.

[70] M. R. Miller and C. S. Petrie "A Multibit Sigma-Delta ADC for Multimode Receivers" IEEE Journal of Solid-State Circuits, Vol. 38., March, 2003, pp. 475482.

[71] G. A. Rincon-Mora, Voltage References ... From diodes to precision higher-order bandgap circuits. Wiley Interscience, IEEE press, 1999, p45-46.

[72] P. R. Gray, P. J. Hurst, S. H. Lewis, and R. G. Meyer, "Analysis and design of analog integrated circuits," 4th edition, New York: John Wiley \& Sons, p146, January 2001.

[73] T. H. Lee, "The design of CMOS radio-frequency integrated circuits" Stanford University, Cambridge University Press, pp395, 1998

[74] Y. L. Guillou, “Analyzing sigma-delta ADCs in deep-submicron CMOS technologies” RF Design, February 2005.

[75] W. Kuang and J. Wight, "A Novel Biquad Filter Circuit for Continuous-Time Sigma-Delta Modulators", IEEE 64 ${ }^{\text {th }}$ Vehicular Technology Conference, Sept. 2006

[76] W. Kuang and J. Wight, "A Novel Biquad Filter Circuit for Continuous-Time Sigma-Delta Modulators", IEEE International Midwest Symposium on Circuits and Systems, Aug. 2006 


\section{Appendix A Quadrature Sampling Alias-Free Conditions}

Let us rewrite Eqs (3.14), (3.15a) and (3.15b) as follows:

$$
\begin{aligned}
& 4\left(f_{c}-f_{1}\right) \leq 1 / T \\
& 4\left(f_{2}-f_{c}\right) \leq 1 / T \\
& T=\frac{k}{2 f_{c}}-\frac{1}{4 f_{c}}
\end{aligned}
$$

where $k$ is a positive integer. Note $1 / T=f_{\mathrm{s}}$. We will prove in the following that if the above equations hold, the general alias-free condition expressed as Eq. (3.12) and rewritten here as Eq. (A.4) also holds.

$$
\left[\operatorname{Int}\left(2 f_{1} / f_{s}\right)+1\right] f_{s} \geq 2 f_{2}
$$

Substituting $T$ with $1 / f_{\mathrm{s}}$ and rearranging Eq. (A.3), we have from Eqs. (A.1), (A.2) and (A.3):

$$
\begin{aligned}
& 4\left(f_{\mathrm{c}}-f_{\mathrm{i}}\right) \leq f_{\mathrm{s}} \\
& 4\left(f_{2}-f_{\mathrm{c}}\right) \leq f_{\mathrm{s}} \\
& f_{c}=\left(\frac{k}{2}-\frac{1}{4}\right) f_{s}
\end{aligned}
$$

Substituting Eq. (A.7) into Eqs. (A.5) and (A.6) gives:

$$
\begin{aligned}
& (2 k-1) f_{\mathrm{s}}-4 f_{\mathrm{i}} \leq f_{\mathrm{s}} \\
& 4 f_{2}-(2 k-1) f_{\mathrm{s}} \leq f_{\mathrm{s}}
\end{aligned}
$$


Rearranging the above equations, we have:

$$
\begin{array}{r}
(k-1) f_{\mathrm{s}} \leq 2 f_{1} \\
k f_{\mathrm{s}} \geq 2 f_{2}
\end{array}
$$

From Eq. (A.10), we have:

$$
k \leq 2 f_{1} / f_{\mathrm{s}}+1
$$

Since $k$ is an integer, Eq. (A.12) is equivalent to:

$$
k \leq \operatorname{Int}\left(2 f_{1} / f_{\mathrm{s}}\right)+1
$$

where $\operatorname{Int}()$ is the integer function. From Eq. (A.11) and (A.13) we have:

$$
\left[\operatorname{Int}\left(2 f_{1} / f_{s}\right)+1\right] f_{s} \geq k f_{s} \geq 2 f_{2}
$$

which is its general alias-free condition.

It can be further shown that the second-order, integer band position, and quadrature sampling is a special case of above sampling. The second-order, integer band position, and quadrature sampling conditions [7][8] are as follows:

$$
\begin{aligned}
& f_{\mathrm{c}}=\left(f_{2}+f_{1}\right) / 2 \\
& 1 / T=2\left(f_{2}-f_{1}\right) \\
& f_{2}=k\left(f_{2}-f_{1}\right)
\end{aligned}
$$

Substituting Eq. (A.15) into (A.16) gives $1 / T=4\left(f_{c}-f_{1}\right)=4\left(f_{2}-f_{c}\right)$, which is the lowest end of Eq. (A.1) and (A.2).

Equations (A.16) and (A.17) give $f_{2}=k / 2 T$. 
Substituting this into $1 / T=4\left(f_{2}-f_{\mathrm{c}}\right)$ yields:

$$
T=\frac{k}{2 f_{c}}-\frac{1}{4 f_{c}}
$$

which is Eq. (A.3). 


\section{Appendix B A $4^{\text {th }}$-order CT Loop Filter Transfer Function}

A quarter-delayed return-to-zero (QRZ) DAC is assumed in the following, the impulse response of which is illustrated below.

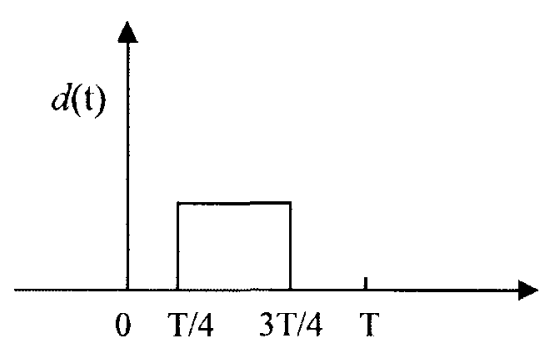

QRZ Zero-order-hold DAC impulse response

Its Laplace transform is:

$$
D(s)=\left(e^{-s T / 4}-e^{-3 s T / 4}\right) / s
$$

It can be shown that the pulse invariant transformation depicted in Eqs. (5.3) and (5.4) gives the following transformation pair (see Appendix C):

$$
\frac{a}{s-j a} \Leftrightarrow=>\frac{e^{j 3 a T / 4}-e^{j a T / 4}}{j} \frac{1}{z-e^{j a T}}
$$

Let $a=\pi / 2 \mathrm{~T}$ in order to have the filter poles located at the quarter sampling frequency. We thus have the following pairs:

$$
\begin{aligned}
& \frac{\frac{\pi}{2 T}}{s-j \frac{\pi}{2 T}}<==>\frac{e^{j 3 \pi / 8}-e^{j \pi / 8}}{j} \frac{1}{z-j} \quad \frac{j}{e^{j 3 \pi / 8}-e^{j \pi / 8}} \frac{1}{S-j}<==>\frac{1}{z-j} \\
& \frac{\frac{\pi}{2 T}}{s+j \frac{\pi}{2 T}} \quad \Leftrightarrow \frac{e^{-j 3 \pi / 8}-e^{-j \pi / 8}}{-j} \frac{1}{z+j} \quad \frac{-j}{e^{-j 3 \pi / 8}-e^{-j \pi / 8}} \frac{1}{S+j} \quad \Longleftrightarrow>\frac{1}{z+j}
\end{aligned}
$$


where the substitution $S=s(2 T / \pi)$ is made for brevity

In the same way we have (from Appendix C):

$\frac{a^{2}}{(s-j a)^{2}} \Leftrightarrow>\left[(1-j 0.75 a T) e^{j 3 a T / 4}+(j 0.25 a T-1) e^{j a T / 4}\right] \frac{1}{z-e^{j a T}}-j a T\left[e^{j 3 a T / 4}-e^{j a T / 4}\right] \frac{e^{j a T}}{\left(z-e^{j a T}\right)^{2}}$

and

$\frac{1}{(S-j)^{2}}<\Rightarrow\left[(1-j 3 \pi / 8) e^{j 3 \pi / 8}+(j \pi / 8-1) e^{j \pi / 8}\right] \frac{1}{z-j}+\frac{\pi}{2}\left[e^{j 3 \pi / 8}-e^{j \pi / 8}\right] \frac{1}{(z-j)^{2}}$

$\frac{1}{(S+j)^{2}}<=\left[(1+j 3 \pi / 8) e^{-j 3 \pi / 8}-(j \pi / 8+1) e^{-j \pi / 8}\right] \frac{1}{z+j}+\frac{\pi}{2}\left[e^{-j 3 \pi / 8}-e^{-j \pi / 8}\right] \frac{1}{(z+j)^{2}}$

The above two pairs can be rewritten as follows:

$$
\begin{aligned}
& \frac{1}{(S-j)^{2}} \Leftrightarrow>\frac{0.3969444+\mathrm{j} 0.4531644}{z-j}+\frac{-0.8501088+\mathrm{j} 0.8501088}{(z-j)^{2}} \\
& \frac{1}{(S+j)^{2}} \Leftrightarrow>\frac{0.3969444-\mathrm{j} 0.4531644}{z+j}+\frac{-0.8501088-j 0.8501088}{(z+j)^{2}}
\end{aligned}
$$

Equations (B.6) and (B.2) can be rearranged as follows:

$$
\begin{aligned}
& (0.14704-\mathrm{j} 0.14704) \frac{1}{(S-j)^{2}} \Leftrightarrow \frac{0.125+\mathrm{j} 0.0082666}{z-j}+\frac{0.25 j}{(z-j)^{2}} \\
& (0.14704+\mathrm{j} 0.14704) \frac{1}{(S+j)^{2}} \Leftrightarrow=\frac{0.125-\mathrm{j} 0.0082666}{z+j}+\frac{-0.25 j}{(z+j)^{2}} \\
& (0.8007573-\mathrm{j} 0.8160319) \frac{1}{S-j} \Leftrightarrow=>\frac{0.875-\mathrm{j} 0.0082666}{z-j} \\
& (0.8007573+\mathrm{j} 0.8160319) \frac{1}{S+j} \Leftrightarrow=\Rightarrow \frac{0.875+\mathrm{j} 0.0082666}{z+j}
\end{aligned}
$$


Adding the above four transformation pairs, we have the following transformation:

$$
\begin{aligned}
& \frac{1}{z+j}+\frac{-0.25 j}{(z+j)^{2}}+\frac{1}{z-j}+\frac{0.25 j}{(z-j)^{2}} \quad \Longleftrightarrow=> \\
& \frac{0.14704}{(S+j)^{2}}+\frac{0.14704}{(S-j)^{2}}+\frac{j 0.14704}{(S+j)^{2}}-\frac{j 0.14704}{(S-j)^{2}}+0.8007573\left[\frac{1}{S+j}+\frac{1}{S-j}\right]+j 0.8160319\left[\frac{1}{S+j}-\frac{1}{S-j}\right]
\end{aligned}
$$

Combining terms yields:

$$
\begin{aligned}
& \frac{2 \mathrm{z}}{z^{2}+1}+\frac{0.25 j 4 j z}{\left(z^{2}+1\right)^{2}}=\frac{2 \mathrm{z}}{z^{2}+1}-\frac{z}{\left(z^{2}+1\right)^{2}}=\frac{2 z^{3}+z}{\left(z^{2}+1\right)^{2}} \quad \Leftrightarrow=> \\
& \quad 0.14704 \frac{2 S^{2}-2}{\left(S^{2}+1\right)^{2}}+j 0.14704 \frac{-j 4 S}{\left(S^{2}+1\right)^{2}}+0.8007573 \frac{2 S}{S^{2}+1}+j 0.8160319 \frac{-2 j}{S^{2}+1} \\
& =\left(0.29408 S^{2}-0.29408+0.58816 \mathrm{~S}+1.601514 S^{3}+1.601514 S+1.632064 S^{2}+1.632064\right) /\left(S^{2}+1\right)^{2} \\
& =\left[1.601514 S^{3}+1.926144 S^{2}+2.189674 S+1.337984\right] /\left(S^{2}+1\right)^{2} \\
& =1.601514\left(S^{3}+1.202702 S^{2}+1.367253 \mathrm{~S}+0.83545\right) /\left(S^{2}+1\right)^{2} \\
& =1.601514\left(S^{2}+0.403159 S+1.04491\right)(\mathrm{S}+0.799543) /\left(S^{2}+1\right)^{2}
\end{aligned}
$$

On the left hand side, $\quad H(z)=\frac{2 z^{3}+z}{\left(z^{2}+1\right)^{2}}=\frac{2 z^{-1}+z^{-3}}{\left(1+z^{-2}\right)^{2}} \quad$ is the popular $4^{\text {th }}$-order loop filter for DT bandpass SDM with one digital delay assigned to the feedback path. 


\section{Appendix C Pulse Invariant Transformation Pair for QRZ DAC}

The QRZ DAC response is:

$$
D(s)=\left(e^{-s T / 4}-e^{-3 . s T / 4}\right) / s
$$

For $\hat{H}(s)=a /(s-j a)$, the overall loop response $H(s)=\hat{H}(s) D(s)$ can be rearranged as:

$$
\begin{aligned}
H(s) & =\left(e^{-s T / 4}-e^{-3 s T / 4}\right) \frac{a}{s(s-j a)}=\frac{\left(e^{-s T / 4}-e^{-3 s T / 4}\right)}{j} \frac{s-(s-j a)}{s(s-j a)} \\
& =\frac{\left(e^{-s T / 4}-e^{-3 s T / 4}\right)}{j}\left[\frac{1}{s-j a}-\frac{1}{s}\right]
\end{aligned}
$$

In the time-domain, this becomes:

$$
h(t)=\frac{1}{j}\left[e^{j a(t-T / 4)} u(t-T / 4)-e^{j a(t-3 T / 4)} u(t-3 T / 4)-u(t-T / 4)+u(t-3 T / 4)\right]
$$

where $U(t)$ is the unit step function. Its sampled form is:

$$
h(n T)=\frac{1}{j}\left[e^{j a T(n-1 / 4)} u(n-1)-e^{j a T(n-3 / 4)} u(n-1)\right]=\frac{e^{j 3 a T / 4}-e^{j a T / 4}}{j a} e^{j a T(n-1)} u(n-1)
$$

whose z-transform is:

$$
H(z)=\frac{e^{j 3 a T / 4}-e^{j a T^{\prime / 4}}}{j} \frac{1}{z-e^{j a T}}
$$

Therefore we have the pulse invariant transformation pair

$$
\frac{a}{s-j a}<==>\frac{e^{j 3 a T / 4}-e^{j a T / 4}}{j} \frac{1}{z-e^{j a T}}
$$


For $\hat{H}(s)=1 /(s-j a)^{2}$

$$
\begin{aligned}
& H(s)=\left(e^{-s T^{\prime} / 4}-e^{-3 s T / 4}\right) \frac{1}{s(s-j a)^{2}}=\frac{\left(e^{-s T / 4}-e^{-3 s T / 4}\right)}{j a} \frac{s-(s-j a)}{s(s-j a)^{2}} \\
& =\frac{\left(e^{-s T / 4}-e^{-3 s T / 4}\right)}{j a}\left[\frac{1}{(s-j a)^{2}}-\frac{1}{s(s-j a)}\right]=\frac{\left(e^{-s / / 4}-e^{-3 s T / 4}\right)}{j a}\left[\frac{1}{(s-j a)^{2}}-\frac{1}{j a} \frac{s-(s-j a)}{s(s-j a)}\right] \\
& =\frac{\left(e^{-s T / 4}-e^{-3 s T / 4}\right)}{j a}\left[\frac{1}{(s-j a)^{2}}-\frac{1}{j a} \frac{1}{s-j a}+\frac{1}{j a} \frac{1}{s}\right] \\
& \begin{array}{l}
h(t)=\frac{1}{j a}\left[(t-T / 4) e^{j a(t-T / 4)} u(t-T / 4)-(t-3 T / 4) e^{j a(t-3 T / 4)} u(t-3 T / 4)\right]- \\
\quad \frac{1}{-a^{2}}\left[e^{j a(t-T / 4)} u(t-T / 4)-e^{j a(t-3 T / 4)} u(t-3 T / 4)-u(t-T / 4)+u(t-3 T / 4)\right]
\end{array} \\
& h(n T)=\frac{T}{j a}\left[(n-1 / 4) e^{j a l(n-1 / 4)}-(n-3 / 4) e^{j a T(n-3 / 4)}\right] u(n-1)+\frac{1}{a^{2}}\left[e^{j a T(n-1 / 4)}-e^{j a T(n-3 / 4)}\right] u(n-1) \\
& =\frac{T}{j a}\left[(n-1+3 / 4) e^{j 3 a T / 4}-(n-1+1 / 4) e^{j a T / 4}\right] e^{j a T(n-1)} u(n-1)+\frac{1}{a^{2}}\left[e^{j 3 a T^{\prime} / 4}-e^{j a T / 4}\right] e^{j a T(n-1)} u(n-1) \\
& =\frac{1}{a^{2}}\left[(1-j 0.75 a T) e^{j 3 a T / 4}+(j 0.25 a T-1) e^{j a T / 4}\right] e^{j a T(n-1)} u(n-1) \\
& +\frac{T}{j a}\left[e^{j 3 a T / 4}-e^{j a T / 4}\right](n-1) e^{j a T(n-1)} u(n-1) \\
& H(z)=\frac{1}{a^{2}}\left[(1-j 0.75 a T) e^{j 3 a T^{\prime} / 4}+(j 0.25 a T-1) e^{j a l / 4}\right] \frac{1}{z-e^{j a T}}+\frac{T}{j a}\left[e^{j 3 a T / 4}-e^{j a T / 4}\right] \frac{e^{j a T}}{\left(z-e^{j a T}\right)^{2}}
\end{aligned}
$$

Therefore the following transformation pair is obtained:

$$
\frac{a^{2}}{(s-j a)^{2}}<=>\left[(1-j 0.75 a T) e^{j a a T / 4}+(j 0.25 a T-1) e^{j a T / 4}\right] \frac{1}{z-e^{j a T}}-j a T\left[e^{j 3 a T / 4}-e^{j a T / 4}\right] \frac{e^{j a T}}{\left(z-e^{j a T}\right)^{2}}
$$

